\title{
Paper folding, Riemann surfaces and convergence of pseudo-Anosov sequences
}

\author{
ANDRÉ DE CARVALHO \\ TOBY HALL
}

\begin{abstract}
A method is presented for constructing closed surfaces out of Euclidean polygons with infinitely many segment identifications along the boundary. The metric on the quotient is identified. A sufficient condition is presented which guarantees that the Euclidean structure on the polygons induces a unique conformal structure on the quotient surface, making it into a closed Riemann surface. In this case, a modulus of continuity for uniformising coordinates is found which depends only on the geometry of the polygons and on the identifications. An application is presented in which a uniform modulus of continuity is obtained for a family of pseudo-Anosov homeomorphisms, making it possible to prove that they converge to a Teichmüller mapping on the Riemann sphere.
\end{abstract}

30C35, 30F10, 37E30; 30C62, 30F45, 37F30

\section{Introduction}

This article addresses the classical problem of constructing surfaces out of subsets of the plane by making identifications along the boundary: in contrast to the usual discussion arising in the classification of surfaces, however, infinitely many identifications are allowed. The topological structure of the identification space $S$ is studied and conditions are given which guarantee that $S$ is a closed surface. The quotient metric on $S$ induced by the Euclidean metric on the plane is identified, and the question of whether or not this metric induces a unique complex structure on $S$ is discussed. A sufficient condition for uniqueness of the complex structure is given and, when it holds, a modulus of continuity for uniformising coordinates is obtained. The interplay between the metric and conformal structures is central to the paper, promoting the topological structure to a Riemann surface structure and providing quantitative control over the quotient map. This analytic control is then used to prove convergence of a certain sequence of pseudo-Anosov homeomorphisms to a generalised pseudo-Anosov homeomorphism.

Let $P$ be a finite collection of disjoint polygons in the (complex) plane. A paper-folding scheme is an equivalence relation which glues together segments - possibly infinitely 
many - along the boundary of $P$. The image of $\partial P$ in the quotient space $S$ is called the scar: it contains cone points, where the total angle is not equal to $2 \pi$, and singular points, such as accumulations of cone points. The following statements summarise the main theorems of this article.

Topological Structure Theorems (Theorems 42 and 50) Necessary and sufficient conditions are given for the quotient space $S$ of a paper-folding scheme to be a closed surface. In particular, if the paper-folding scheme is plain ( $P$ is a single polygon and the identifications along its boundary are unlinked) then $S$ is a topological sphere and the scar is a dendrite.

Metric Structure Theorem (Theorem 56) The quotient metric on $S$ is intrinsic, and $S$ is a conic-flat surface (that is, it is locally isometric to cones on circles) away from singular points.

Conformal Structure Theorem (Theorem 59) The natural conformal structure on the conic-flat part of $S$ extends uniquely across an isolated singularity provided that a certain integral diverges. In particular, if there are only finitely many singular points at each of which the relevant integral diverges, then the conformal structure extends uniquely across the singular set, making $S$ into a closed Riemann surface.

It is possible to extend this theorem to the case of arbitrary singular sets, and this will be the subject of a forthcoming paper.

If the conditions of both Theorem 50 and Theorem 59 are satisfied, then $S$ has a natural closed Riemann surface structure. If $S$ is a topological sphere, as is the case for plain paper foldings, then $S$ is isomorphic to the Riemann sphere. A modulus of continuity for a suitably normalised uniformising map from the polygon to the Riemann sphere is found and the following theorem is proved:

Modulus of continuity of uniformising map (Theorem 84) The uniformising map has a modulus of continuity which depends only on the geometry of the polygon and on the metric on the scar.

The theorems above belong to the fields of surface topology, Riemann surface theory and geometric function theory. The question that motivated this work, however, comes from dynamical systems theory and was described to the first author many years ago by Dennis Sullivan. Consider a self-homeomorphism of a surface, such as the much-studied Hénon diffeomorphism of the plane

$$
f(x, y):=\left(a-x^{2}-b y, x\right),
$$


where $a, b$ are real parameters. There is a large set of parameters for which $f$ is chaotic which means, amongst other things, that it has infinitely many periodic orbits. If $Q$ is a finite $f$-invariant set (a union of periodic orbits), consider the isotopy class of $f$ in the punctured plane $\mathbb{R}^{2} \backslash Q$. Thurston's classification of surface homeomorphisms provides a canonical representative $\varphi_{Q}$ in this isotopy class, which is, typically, a pseudo-Anosov homeomorphism. If $Q_{n}$ is an increasing sequence which exhausts the collection of finite $f$-invariant sets, one can imagine passing to the limit in the sequence $\varphi_{Q_{n}}$ of associated pseudo-Anosov maps. The resulting limit would be a tight representative of $f$ which contains 'all' of its dynamics. The paper ends with an application of the theorems above to establish a case of this scenario:

Convergence to the tight horseshoe (Theorem 105) There exists a cofinal sequence in the set of horseshoe braid types partially ordered by forcing whose associated pseudoAnosov homeomorphisms converge to the tight horseshoe.

To understand the existence of limits of sequences of pseudo-Anosov maps on a fixed surface $S$ relative to varying finite subsets $Q$ has several consequences. From a purely intrinsic point of view, it is interesting to be able to describe the closure of the set of relative pseudo-Anosov maps on $S$ - an important class of homeomorphisms - in the space of all self-homeomorphisms of $S$. Also, Thurston's classification of surface homeomorphisms up to isotopy produces geometrically and conformally rigid structures out of the topology. Understanding limits of pseudo-Anosovs would do the same in a more general context and, in this case, the dynamics and not the topology would be the driving force. It is hoped that this will eventually lead to the correct 2-dimensional analogue of the Milnor-Thurston theorem stating that every multimodal interval map has a piecewise-linear quotient with same entropy. There are also connections to be explored with infinite dimensional Teichmüller theory, since the tight limits are Teichmüller mappings with respect to quadratic differentials in the $L^{1}$-closure of the set of meromorphic quadratic differentials.

Section 2 provides a brief summary of results from surface topology, metric geometry and geometric function theory which will be used in the remainder of the paper. In Section 3 the main objects of study, paper-folding schemes, are defined. These are identification schemes around the boundaries of finite disjoint unions of polygons, whose (metric) quotients are called paper spaces.

Sections 4 and 5 contain discussions of the topological, metric and conformal structures of paper spaces. To simplify the exposition, some of the arguments are first developed in the simplest case of plain paper foldings, before being extended to the general case.

In Section 6, moduli of continuity are discussed, first locally for uniformising coordinate charts on the neighborhood of points on $S$ at which the conformal structure extends 
uniquely and then globally in the case where $S$ is a complex sphere and is thus isomorphic to the Riemann sphere.

In Section 7, the dynamical application is given. A sequence of pseudo-Anosov maps of the punctured sphere is considered: each can be regarded as a quasi-conformal homeomorphism of an appropriate (finite) paper surface. Using the results of Section 6, the natural limit of this sequence is constructed. In fact this is equivalent to taking the limit of pseudo-Anosov maps over an exhaustion of the collection of finite invariant sets of Smale's horseshoe map.

A brief account of the results described in this article, their application and an outline of the methods of proof can be found in [7].

Acknowledgements The authors would like to thank Lasse Rempe for his help with the proof of Lemma 103 and Phil Boyland for suggestions for improving the text and many encouraging conversations. They gratefully acknowledge the support of FAPESP grant 2006/03829-2. The first author would also like to acknowledge the support of CNPq grant 151449/2008-2 and the hospitality of IMPA, where part of this work was developed.

\section{Background}

This section contains a summary of background theory which will be used in the article, together with references to more detailed accounts. Section 2.1 contains topological results which will be used in Section 4 to give conditions under which the quotient space associated with a paper-folding scheme is a topological sphere or, more generally, a surface. The main tool here is Moore's theorem [14; 15], which provides conditions under which the quotient of a sphere by an equivalence relation is again a sphere. Section 2.2 contains a brief summary of the theory of quotient metric spaces and their relationship to topological quotients. Finally, Section 2.3 describes some results from geometric function theory which will be used in Section 5 to give conditions under which the conformal structure on the quotient space extends uniquely across an isolated singularity; and in Section 6 to determine a modulus of continuity for a uniformising map from the polygon to the Riemann sphere in the case of a plain paper folding.

\subsection{Moore's theorem and the dendrite quotient theorem}

Definitions 1 (Separation, continuum) A separation of a topological space $X$ is a decomposition of $X$ as a disjoint union $X=A \cup B$ of non-empty closed subsets of $X$. The space $X$ is connected if no separation of $X$ exists. 
A set $C \subset X$ separates two points $x, y \in X$ (respectively subsets $D, E \subset X$ ) if there is a separation $X \backslash C=A \cup B$ with $x \in A, y \in B$ (respectively $D \subset A, E \subset B$ ). If $X$ is connected and $X \backslash C$ is not, $C$ separates $X$.

A continuum is a compact connected (subset of a) Hausdorff topological space.

Definitions 2 (Monotone upper semi-continuous decomposition) An equivalence relation $\sim$ on a topological space $X$ is closed if it is closed as a subset of $X \times X$. (On compact Hausdorff spaces, being closed is the same as saying that $x_{n} \sim y_{n}, x_{n} \rightarrow x$ and $y_{n} \rightarrow y$ imply $x \sim y$; and an equivalence relation is closed if and only if the quotient space is Hausdorff.)

A decomposition of $X$ is synonymous with a partition of $X$, that is, a collection of disjoint subsets whose union is $X$. A decomposition $\mathcal{G}$ of a topological space into compact subsets is upper semi-continuous (usc) if the associated equivalence relation is closed. A decomposition is monotone if its elements are connected.

The expression "monotone upper semi-continuous" will be abbreviated musc.

Definition 3 (Realising an equivalence relation) Let $P$ be a subset of a topological space $X$, and $\sim$ be an equivalence relation on $P$. A decomposition $\mathcal{G}$ of $X$ realises $\sim$ if all elements of $\mathcal{G}$ intersect $P$, and each intersection of an element of $\mathcal{G}$ with $P$ is a $\sim$-equivalence class. (This means that $P / \sim$ is naturally identified with $X / \mathcal{G}$.)

Definition 4 (Saturation) Let $\mathrm{R}$ be a reflexive and symmetric relation on a set $X$. A subset $U$ of $X$ is $\mathrm{R}$-saturated if it contains $\{y \in X: y \mathrm{R} x\}$ for all $x \in U$.

Any collection $\mathcal{P}$ of subsets of $X$ (not necessarily a partition) naturally defines a symmetric and reflexive relation $\mathrm{R}$ (two distinct points are related if they belong to the same element of $\mathcal{P}$ ), which is transitive if the elements of $\mathcal{P}$ are disjoint. A subset $U$ of $X$ is $\mathcal{P}$-saturated if it is $\mathrm{R}$-saturated.

The following theorem, due to Moore [14], is the main tool used for deciding when the quotient of an equivalence relation on the $2-$ sphere is again the sphere. A generalisation for surfaces was given by Roberts and Steenrod [16].

Theorem 5 (Moore on the 2-sphere) A topological quotient of the 2-sphere by a musc decomposition whose elements do not separate it is again homeomorphic to the 2-sphere.

The topological structure of the scar of a paper-folding scheme will also be important, and the main tool which will be used in this regard is Theorem 7 below. 
Definitions 6 (Dendrite, dendritic collection) A dendrite is a locally connected continuum which does not contain any simple closed curve. A local dendrite is a continuum which is locally a dendrite, that is, for which every point has a (closed) neighborhood which is a dendrite.

A collection $\mathcal{G}$ of disjoint subsets of a topological space $X$ is non-separated if no element of $\mathcal{G}$ separates two points of any other single element of $\mathcal{G}$. A non-separated collection $\mathcal{G}$ is dendritic if given $g \in \mathcal{G}$ and $y \notin g$, there exists $g^{\prime} \in \mathcal{G}$ which separates $y$ and $g$.

The following theorem can be found in Whyburn [19]. There the word saturated is used to signify what was defined as dendritic above (the former word has already been used here).

Theorem 7 (Dendrite quotient) Let $\mathcal{G}$ be a dendritic decomposition of a continuum $X$. Then $\mathcal{G}$ is usc and the quotient space $X / \mathcal{G}$ is a dendrite.

Here is a list of properties of dendrites which will be used later. The main references for them are Kuratowski [11] and Whyburn [19].

Theorem 8 (Properties of dendrites)

(a) Any two distinct points in a dendrite $G$ are separated by a third point in $G$. Conversely, any continuum with this property is a dendrite.

(b) If $G$ is a dendrite and $x, y \in G$, there exists a unique $\operatorname{arc} \gamma \subset G$ whose endpoints are $x, y$. The notation $[x, y]_{G}$ will be used to indicate this arc and expressions such as $(x, y]_{G}$ will have the usual meaning (that is, $\left.(x, y]_{G}=[x, y]_{G} \backslash\{x\}\right)$.

(c) A point $p$ in a continuum $G$ is an endpoint if it has arbitrarily small neighborhoods (in $G$ ) whose boundary is a single point; and it is a cut point if $G \backslash\{p\}$ is disconnected. A continuum is a dendrite if and only if all of its points are either endpoints or cut points.

(d) Dendrites are contractible (see Fort [9]).

(e) Every subcontinuum of a dendrite is also a dendrite. (A subcontinuum of a continuum $G$ is a subset which is also a continuum.)

(f) Every connected subset of a dendrite is arcwise connected, and the intersection of any two connected subsets is connected. 


\subsection{Quotient metric spaces and intrinsic metrics}

The quotient spaces associated with paper-folding schemes are metric rather than topological quotients, and this section summarises some relevant definitions and results. Burago, Burago and Ivanov [3] is an excellent reference for readers seeking further details.

Definitions 9 (Metric and semi-metric) A metric on a set $X$ is a function $d_{X}: X \times$ $X \rightarrow \mathbb{R} \cup\{+\infty\}$ satisfying the usual conditions of positivity, symmetry and the triangle inequality (it is convenient to allow two points to be infinitely distant from one another). When no ambiguity arises, subscripts may be dropped so that, for example, the usual Euclidean distance on $\mathbb{R}^{2}$ or $\mathbb{C}$ may be denoted $d_{\mathbb{R}^{2}}(x, y), d_{\mathbb{C}}(x, y), d(x, y)$, or even $|x y|$.

A semi-metric on a set $X$ satisfies the same axioms as a metric, except that distinct points are allowed to be at semi-distance zero from one another.

Notation 10 The following notation is used for metric and semi-metric spaces $\left(X, d_{X}\right)$. If $x \in X$ and $r \geq 0$, then

$$
\begin{aligned}
B_{X}(x ; r) & :=\left\{y \in X: d_{X}(y, x)<r\right\}, \\
& \bar{B}_{X}(x ; r):=\left\{y \in X: d_{X}(y, x) \leq r\right\}, \\
\text { and } \quad & C_{X}(x ; r):=\left\{y \in X: d_{X}(y, x)=r\right\} .
\end{aligned}
$$

If ambiguity lurks, subscripts may be enhanced so that $B_{X}(x ; r)$, for example, may be denoted $B_{\left(X, d_{X}\right)}(x ; r)$ or $B_{d_{X}}(x ; r)$. At the other extreme, if the ambient space is clear, $B(x ; r)$ may be used.

If $x \in X$ and $A \subset X$, the distance from $x$ to $A$ is

$$
d_{X}(x, A):=\inf \left\{d_{X}(x, y): y \in A\right\} .
$$

and if $A, B \subset X$, the distance between them is

$$
d_{X}(A, B):=\inf \left\{d_{X}(x, y): x \in A, y \in B\right\} .
$$

Given a set $A \subset X$, the sets $B_{X}(A ; r), \bar{B}_{X}(A ; r)$ and $C_{X}(A ; r)$ are defined by substituting $d(y, A)$ for $d(y, x)$ in (1).

Definitions 11 (Quotient metric) Let $(X, d)$ be a metric space and $\mathrm{R}$ be a reflexive and symmetric relation on $X$ (which in this article will usually arise from a collection $\mathcal{P}$ of subsets of $X$, as in Definition 4). An $\mathrm{R}$-chain from $x$ to $y$ is a sequence 
$\left(\left(p_{i}, q_{i}\right)\right)_{i=0}^{k}$ in $X^{2}$ such that $x \mathrm{R} p_{0}, q_{i} \mathrm{R} p_{i+1}$ for $i=0, \ldots, k-1$, and $q_{k} \mathrm{R} y$. Its length is

$$
\mathrm{L}^{\mathrm{R}}\left(\left(p_{i}, q_{i}\right)\right):=\sum_{i=0}^{k} d\left(p_{i}, q_{i}\right)
$$

(thus one pays to move between unrelated elements of $X$, but moving between related elements is free).

Define $d^{\mathrm{R}}: X \times X \rightarrow \mathbb{R}_{\geq 0} \cup\{\infty\}$ by

$$
d^{\mathrm{R}}(x, y):=\inf \left\{\mathrm{L}^{\mathrm{R}}\left(\left(p_{i}, q_{i}\right)\right):\left(\left(p_{i}, q_{i}\right)\right) \text { is an } \mathrm{R} \text {-chain from } x \text { to } y\right\} .
$$

Then $d^{\mathrm{R}}$ is a semi-metric on $X$. The equivalence relation which identifies points at $d^{\mathrm{R}}$-semi-distance 0 is denoted $\sim_{\mathrm{R}}$ and the quotient space under this equivalence relation is the quotient metric space of $(X, d)$ under the relation $\mathrm{R}$, denoted either $\left(X / d^{\mathrm{R}}, d^{\mathrm{R}}\right)$ or $\left(X / \sim_{\mathrm{R}}, d^{\mathrm{R}}\right)$. (So the same symbol $d^{\mathrm{R}}$ is used to denote both the semi-metric and the metric on the quotient space.)

Many mathematicians, including the authors, are more familiar with topological quotients. Assuming that $\mathrm{R}$ is an equivalence relation, there are essentially two reasons why the quotient metric space $\left(X / d^{\mathrm{R}}, d^{\mathrm{R}}\right)$ and the topological quotient $X / \mathrm{R}$ may differ. First, they may differ as sets: for example, the metric quotient of $\mathbb{R}$ by $\mathbb{Q}$ is a point (as is the metric quotient of any metric space by a relation $\mathrm{R}$ which identifies a dense set of points), since there are arbitrarily short $\mathrm{R}$-chains joining any two points. Second, even if the two quotients agree as sets, they may have different topologies. An instructive example [3, Example 3.1.17] is the following. Let $X$ be a disjoint union of countably many intervals $I_{i}$, of lengths $\ell_{i}$, and consider the quotients by the relation which identifies the left hand endpoints of all of the intervals. The topology of the metric quotient depends on the lengths $\ell_{i}$ : in particular, it is compact if $\ell_{i} \rightarrow 0$, and non-compact otherwise. Clearly, though, the topological quotient does not depend on these lengths.

The case where $\ell_{i} \rightarrow 0$ will be of interest later:

Definition $12(\infty$-od) An $\infty$-od is the metric quotient of countably many intervals $I_{i}$ of lengths $\ell_{i}$, with $\ell_{i} \rightarrow 0$ as $i \rightarrow \infty$, under the relation which identifies all of their left hand endpoints.

The problem of having quotients which agree as sets but not as topological spaces does not arise if $X$ is compact (see Burago, Burago and Ivanov [3]): 
Theorem 13 (Metric and topological quotients) Let $X$ be a compact metric space, and $\mathrm{R}$ be a reflexive and symmetric relation on $X$. Then the topological quotient $X / \sim_{\mathrm{R}}$ and the metric quotient $\left(X / \sim_{\mathrm{R}}, d^{\mathrm{R}}\right)$ are homeomorphic.

(Notice that in this statement the two quotients are equal as sets by definition, since the same equivalence relation $\sim_{R}$ is used in both cases.)

The quotient associated to a paper-folding scheme on a polygon $P$ is constructed by taking the metric quotient of $P$ by a relation $\mathrm{R}$ determined by the folding scheme. Since $P$ is compact, this metric quotient is homeomorphic to the topological quotient $P / \sim_{\mathrm{R}}$, which will be studied using Moore's theorem.

In this article subsets of metric spaces will normally be endowed not with the subspace metric, but with the intrinsic metric inherited from the parent space. The remainder of this section provides a brief summary of these ideas.

Definitions 14 (Length and intrinsic metric) Let $(X, d)$ be a metric space. A path in $\mathrm{X}$ is a continuous map $\gamma:[a, b] \rightarrow X$. The length of the path $\gamma$ is

$$
|\gamma|_{X}=\sup \left\{\sum d\left(\gamma\left(t_{i}\right), \gamma\left(t_{i+1}\right)\right)\right\} \in \mathbb{R}_{\geq 0} \cup\{\infty\},
$$

where the supremum is taken over all finite partitions $a=t_{0}<t_{1}<\cdots<t_{k}=b$ of the interval $[a, b]$. When the metric space $X$ is clear from the context, the length will be denoted $|\gamma|$ without the subscript. If $\gamma$ is injective, then the length of the image of $\gamma$ is defined to be $|\operatorname{im}(\gamma)|_{X}:=|\gamma|_{X}$.

A path is rectifiable if its length is finite.

A metric is intrinsic if the distance between any two points is arbitrarily well approximated by lengths of curves joining the two points, that is,

$$
d(x, y)=\inf \{|\gamma|: \gamma:[a, b] \rightarrow X \text { is a path with } \gamma(a)=x, \gamma(b)=y\} .
$$

The metric is strictly intrinsic if the infimum is attained, that is, if for every $x, y \in X$, there exists a continuous path from $x$ to $y$ whose length equals $d(x, y)$. (In particular, $d(x, y)=+\infty$ if $x$ and $y$ lie in different path components of $X$.) It can be shown (see Burago, Burago and Ivanov [3]) that a complete locally compact intrinsic metric is strictly intrinsic. In particular:

Theorem 15 A compact intrinsic metric is strictly intrinsic.

If $d$ is not an intrinsic metric, then there is an induced intrinsic metric $\hat{d}$ on $X$, defined by

$$
\widehat{d}(x, y)=\inf \{|\gamma|: \gamma:[a, b] \rightarrow X \text { is a path with } \gamma(a)=x, \gamma(b)=y\}
$$


(that $\hat{d}$ is an intrinsic metric follows from the straightforward observation that the length $|\gamma|$ doesn't depend on whether the metric $d$ or $\hat{d}$ is used). For example, if $P \subset \mathbb{R}^{2}$ is a polygon, then there is an intrinsic metric $d_{P}$ on $P$ induced by the metric on $\mathbb{R}^{2}$, which does not agree with the subspace metric on $P$ unless $P$ is convex.

A proof of the following result can be found in [3, Pages 62-63].

Lemma 16 Let $(X, d)$ be an intrinsic metric space, and $\mathrm{R}$ be a reflexive and symmetric relation on $X$. Then the quotient metric space $\left(X / d^{\mathrm{R}}, d^{\mathrm{R}}\right)$ is also intrinsic.

Remark 17 The projection $\pi$ from a metric space $X$ to a metric quotient $X / d^{\mathrm{R}}$ does not increase the distances between points or the lengths of paths. That is, if $x, y \in X$ then $d^{\mathrm{R}}(\pi(x), \pi(y)) \leq d(x, y)$; and if $\gamma$ is a path in $X$, then $|\pi \circ \gamma|_{X / d^{\mathrm{R}}} \leq|\gamma|_{X}$.

The following three results about Hausdorff 1 -dimensional measure $\mu_{X}^{1}$ on a metric space $X$ are [3, Lemma 2.6.1 and Theorem 2.6.2] and a corollary of [17, Theorem 29] respectively.

Lemma 18 If $X$ is a connected metric space, then $\mu_{X}^{1}(X) \geq \operatorname{diam} X$.

Lemma 19 If $\gamma:[a, b] \rightarrow X$ is a rectifiable simple path, then $|\gamma|_{X}=\mu_{X}^{1}(\gamma([a, b]))$.

Lemma 20 Let $(X, \rho)$ and $(Y, \sigma)$ be metric spaces, and $f: X \rightarrow Y$ be a function with $\sigma(f(x), f(y)) \leq \rho(x, y)$ for all $x, y \in X$. Then $\mu_{Y}^{1}(f(A)) \leq \mu_{X}^{1}(A)$ for every $\mu_{X}^{1}$-measurable subset $A$ of $X$.

\subsection{Background geometric function theory}

In this section some results from geometric function theory which will be used in Sections 5 and 6 are summarised. Standard references are Ahlfors-Sario [2], Ahlfors [1] and Lehto-Virtanen [12].

Definitions 21 (Module of an annular region, concentric, nested) An annular region in a surface is a subset homeomorphic to an open round annulus

$$
A\left(r_{1}, r_{2}\right)=\left\{z \in \mathbb{C}: r_{1}<|z|<r_{2}\right\},
$$

where $0 \leq r_{1}<r_{2} \leq \infty$. If $R \subset \mathbb{C}$ is an annular region, then there is a conformal map taking $R$ onto some round annulus $A\left(r_{1}, r_{2}\right)$, and this map is unique up to 
postcomposition with a homothety. It follows that the ratio $r_{2} / r_{1}$ is a conformal invariant of $R$, and the module $\bmod R$ of $R$ is defined by

$$
\bmod R= \begin{cases}\infty & \text { if } r_{1}=0 \text { or } r_{2}=\infty, \\ \frac{1}{2 \pi} \ln \frac{r_{2}}{r_{1}} & \text { otherwise. }\end{cases}
$$

Suppose that $S$ is a closed topological disk and that $R \subset S$ is an annular region. The bounded component of $S \backslash R$ is the one which is disjoint from $\partial S$. Annular regions $R_{0}, R_{1} \subset S$ are concentric if the bounded complementary component of one of them is contained in the bounded complementary component of the other. They are nested if they are concentric and disjoint, that is, if one is entirely contained in the bounded complementary component of the other.

If $R_{0}, R_{1}$ are concentric annular regions and $R_{1} \subset R_{0}$, then $\bmod R_{1} \leq \bmod R_{0}$. The way in which this observation will be used here is in the form of the following:

Lemma 22 (Conformal puncture) Consider a 1-parameter family $R(t)(t \geq 0)$ of concentric annular regions contained in a disk in the complex plane. Assume each $R(t)$ has finite module and that $R\left(t^{\prime}\right) \subset R(t)$ if $t<t^{\prime}$. If $\bmod R(t) \rightarrow \infty$ as $t \downarrow 0$ then $R(0)=\bigcup_{t>0} R(t)$ is an open annular region whose bounded complementary component is a point.

To verify the hypotheses of this lemma, the following fundamental inequality will be used.

Lemma 23 If $\left\{R_{n}\right\}$ is a finite or countable family of nested annular regions all contained in and concentric with the annular region $R$, then

$$
\bmod R \geq \sum \bmod R_{n}
$$

From this it follows that if $\sum \bmod R_{n}$ diverges then at least one of the complementary components of $R$ is a point.

A conformal metric on $\mathbb{C}$ is a metric obtained defining the length of arcs by $|\gamma|_{\nu}:=$ $\int_{\gamma} v(z)|\mathrm{d} z|$, where $v$ is a nonnegative Borel measurable function defined on $\mathbb{C}$. If $R$ is an annular region in $\mathbb{C}$ whose boundary components are $C_{1}, C_{2}$ then

$$
\bmod R=\sup \left\{\frac{d_{v}\left(C_{1}, C_{2}\right)^{2}}{\operatorname{Area}_{v}(R)}: v(z)|\mathrm{d} z| \text { is a conformal metric }\right\},
$$


where $d_{v}\left(C_{1}, C_{2}\right)$ is the $v$-distance between the boundary components of $R$ (that is, the minimum $v$-length of an arc with endpoints in $C_{1}$ and $\left.C_{2}\right)$ and $\operatorname{Area}_{v}(R)=$ $\iint_{R} v(z)^{2} \mathrm{~d} x \mathrm{~d} y$ is the $v$-area of $R$. It follows that

$$
\bmod R \geq \frac{d_{v}\left(C_{1}, C_{2}\right)^{2}}{\operatorname{Area}_{v}(R)}
$$

for any conformal metric $v(z)|\mathrm{d} z|$.

Definition 24 (Grötzsch annular region) Let $t \in[0,1)$. The Grötzsch annular region $\operatorname{Gr}(t)$ is the region obtained from the open unit disk by removing the closed interval $[0, t]$ in the real axis.

The following inequality can be found in Lehto-Virtanen [12] and Ahlfors [1]:

$$
\bmod \operatorname{Gr}(t) \leq \frac{1}{2 \pi} \ln \frac{4}{t}
$$

The following theorem (see [1, Page 72]) will be of fundamental importance in Section 6.

Theorem 25 (Grötzsch Annulus Theorem) Let $R$ be an annular region contained in the unit disk in the complex plane. If both 0 and a point $z$ are contained in the bounded complementary component of $R$, then

$$
\bmod R \leq \bmod \operatorname{Gr}(|z|) \leq \frac{1}{2 \pi} \ln \frac{4}{|z|} .
$$

The following distortion theorem will be needed. For completeness, a proof is included. The term univalent is used in this article as a synonym for injective holomorphic.

Theorem 26 Let $P \subset \mathbb{C}$ be a closed topological disk, $\tilde{p} \in \operatorname{Int}(P)$ and $\Phi: \operatorname{Int}(P) \rightarrow \mathbb{C}$ be a univalent function with $\Phi(\widetilde{p})=0$ and $\Phi^{\prime}(\widetilde{p})=1$. Let $\widetilde{Q}(h)$ denote the closed interior collar neighborhood of $\partial P$ of $d_{\mathbb{C}}$-width $h>0$ and set $P_{h}:=P \backslash \widetilde{Q}(h)$. Assume that $h$ is small enough that $P_{h}$ is path connected and $\tilde{p} \in P_{h}$, and set

$$
\kappa:=\exp \left(\frac{8 \operatorname{diam}_{P_{h}} P_{h}}{h}\right),
$$

where diam $P_{h} P_{h}$ denotes the diameter of $P_{h}$ in the intrinsic metric $d_{P_{h}}$ on $P_{h}$ induced by $d_{\mathbb{C}}$. Then for any $\tilde{q} \in P_{h}$,

$$
\frac{1}{\kappa} \leq\left|\Phi^{\prime}(\widetilde{q})\right| \leq \kappa
$$

In particular, $\Phi$ is $\kappa$-biLipschitz in $P_{h}$. 
Proof The usual distortion theorem (see Ahlfors [1]) states that if $f: \mathbb{D} \rightarrow \mathbb{C}$ is univalent then, for any $z_{1}, z_{2} \in \mathbb{D}$,

$$
\frac{1}{m\left(z_{2}, z_{1}\right)} \leq \frac{\left|f^{\prime}\left(z_{1}\right)\right|}{\left|f^{\prime}\left(z_{2}\right)\right|} \leq m\left(z_{1}, z_{2}\right)
$$

where

$$
m\left(z_{1}, z_{2}\right):=\frac{1+\left|z_{1}\right|}{1-\left|z_{2}\right|} \cdot\left(\frac{1+\left|z_{2}\right|}{1-\left|z_{1}\right|}\right)^{3} .
$$

Let $\zeta: \mathbb{D} \rightarrow \operatorname{Int}(P)$ be a Riemann mapping with $\zeta(0)=\tilde{p}$ and set $f:=\Phi \circ \zeta$. Then both $f$ and $\zeta$ are univalent so that, writing $\zeta_{i}:=\zeta\left(z_{i}\right)$ for $i=1,2$, it follows from (5) that

$$
\frac{1}{m\left(z_{1}, z_{2}\right) \cdot m\left(z_{2}, z_{1}\right)} \leq \frac{\left|\Phi^{\prime}\left(\zeta_{1}\right)\right|}{\left|\Phi^{\prime}\left(\zeta_{2}\right)\right|}=\frac{\left|f^{\prime}\left(z_{1}\right)\right|}{\left|f^{\prime}\left(z_{2}\right)\right|} \cdot \frac{\left|\zeta^{\prime}\left(z_{2}\right)\right|}{\left|\zeta^{\prime}\left(z_{1}\right)\right|} \leq m\left(z_{1}, z_{2}\right) \cdot m\left(z_{2}, z_{1}\right)
$$

and (6) gives

$m\left(z_{1}, z_{2}\right) \cdot m\left(z_{2}, z_{1}\right)=\left(\frac{1+\left|z_{1}\right|}{1-\left|z_{1}\right|} \cdot \frac{1+\left|z_{2}\right|}{1-\left|z_{2}\right|}\right)^{4}=\left[\exp \left(d_{\mathbb{D}}^{H}\left(0, z_{1}\right)\right) \cdot \exp \left(d_{\mathbb{D}}^{H}\left(0, z_{2}\right)\right)\right]^{4}$,

where $d_{\mathbb{D}}^{H}$ denotes the Poincaré distance in the unit disk $\mathbb{D}$. Setting $z_{2}=0$ it follows that, for any $z \in \mathbb{D}$,

$$
\left[\exp \left(d_{\mathbb{D}}^{H}(0, z)\right)\right]^{-4} \leq\left|\Phi^{\prime}(\zeta(z))\right| \leq\left[\exp \left(d_{\mathbb{D}}^{H}(0, z)\right)\right]^{4} .
$$

Now let $\lambda_{P}(\zeta)|d \zeta|$ denote the Poincaré metric on $\operatorname{Int}(P)$. It is well known (see for example [1]) that

$$
\lambda_{P}(\zeta) \leq \frac{2}{d_{\mathbb{C}}(\zeta, \partial P)} .
$$

Let $\tilde{q}=\zeta(z) \in P_{h}$. Then, if $d_{P}^{H}$ denotes the Poincaré distance in $\operatorname{Int}(P)$,

$$
\begin{aligned}
d_{\mathbb{D}}^{H}(0, z) & =d_{P}^{H}(\tilde{p}, \widetilde{q}) \\
& =\inf \left\{\int_{\gamma} \lambda_{P}(\zeta)|\mathrm{d} \zeta| ; \gamma \text { is a path } \operatorname{in} \operatorname{Int}(P) \text { from } \tilde{p} \text { to } \widetilde{q}\right\} \\
& \leq \inf \left\{\int_{\gamma} \lambda_{P}(\zeta)|\mathrm{d} \zeta| ; \gamma \text { is a path in } P_{h} \text { from } \widetilde{p} \text { to } \widetilde{q}\right\} \\
& \leq \frac{2 d_{P_{h}}(\tilde{p}, \widetilde{q})}{h} \\
& \leq \frac{2 \operatorname{diam}_{P_{h}} P_{h}}{h}
\end{aligned}
$$

which, together with (7), establishes the result. 
Finally, the following holomorphic removability criterion will be needed: it is a consequence of [12, Theorem V.3.2, page 202].

Theorem 27 Let $\Omega \subset \mathbb{C}$ be a domain and $E \subset \Omega$ be a compact subset with finite 1-dimensional Hausdorff measure. Suppose $g: \Omega \rightarrow \mathbb{C}$ is an orientation-preserving homeomorphism onto its image which is conformal on $\Omega \backslash E$. Then, in fact, $g$ is conformal on all of $\Omega$.

\section{Paper-folding schemes}

This section provides the definition of paper-folding schemes, which are the main objects of study in this article. Some properties of the metric and measure on the scar of a paper-folding scheme are established (Lemma 33), and the notion of a plain paper-folding scheme is introduced.

The polygons used in the definition of paper-folding schemes are subsets of the Euclidean plane $\mathbb{R}^{2}$, which will be identified with the complex plane $\mathbb{C}$ in Sections 5 and 6 when the complex structure of paper surfaces is discussed. The notation $\widehat{\mathbb{C}}$ will be used for the Riemann sphere.

Definitions 28 (Arc, segment, polygon, multipolygon) An arc in a metric space $X$ is a homeomorphic image $\gamma \subset X$ of the interval [0,1]. Its endpoints are the images of 0 and 1 and its interior is the image of $(0,1)$, denoted $\dot{\gamma}$. An open arc is the interior of an arc. A segment is an arc in $\mathbb{R}^{2}$ which is a subset of a straight line. The length of a segment $\alpha$ is denoted $|\alpha|$. A simple closed curve in $X$ is a homeomorphic image of the unit circle.

An arc or simple closed curve in $\mathbb{R}^{2}$ is called polygonal if it is the concatenation of finitely many segments. Its vertices are the intersections of consecutive maximal segments, and the maximal segments themselves are its edges.

A polygon is a closed topological disk in $\mathbb{R}^{2}$ whose boundary is a polygonal simple closed curve. Its vertices are the same as its boundary's vertices and its sides are the edges forming its boundary.

A multipolygon is a disjoint union of finitely many polygons. A (polygonal) multicurve is a disjoint union of finitely many (polygonal) simple closed curves.

Definitions 29 (Segment pairing, interior pair, full collection, fold) Let $C \subset \mathbb{R}^{2}$ be an oriented polygonal multicurve and $\alpha, \alpha^{\prime} \subset C$ be segments of the same length with disjoint interiors. The segment pairing $\left\langle\alpha, \alpha^{\prime}\right\rangle$ is the relation which identifies 
pairs of points of $\alpha$ and $\alpha^{\prime}$ in a length-preserving and orientation-reversing way. The segments $\alpha, \alpha^{\prime}$ and any two points which are identified under the pairing are said to be paired. Two paired points which lie in the interior of a segment pairing form an interior pair. Observe that the notation for a pairing is not ordered, so that $\left\langle\alpha, \alpha^{\prime}\right\rangle$ and $\left\langle\alpha^{\prime}, \alpha\right\rangle$ represent the same pairing.

A collection $\left\{\left\langle\alpha_{i}, \alpha_{i}^{\prime}\right\rangle\right\}$ of segment pairings is interior disjoint if the interiors of all of the segments $\alpha_{i}$ and $\alpha_{i}^{\prime}$ are pairwise disjoint.

The length of a segment pairing $\left\langle\alpha, \alpha^{\prime}\right\rangle$, denoted $\left|\left\langle\alpha, \alpha^{\prime}\right\rangle\right|$, is the length of one of the arcs in the pairing, that is, $\left|\left\langle\alpha, \alpha^{\prime}\right\rangle\right|:=|\alpha|=\left|\alpha^{\prime}\right|$. If $\mathcal{P}=\left\{\left\langle\alpha_{i}, \alpha_{i}^{\prime}\right\rangle\right\}$ is a (countable) interior disjoint collection of segment pairings on $C$, its length, denoted $|\mathcal{P}|$, is the sum of the lengths of the pairings in $\mathcal{P}$, that is, $|\mathcal{P}|=\sum_{i}\left|\left\langle\alpha_{i}, \alpha_{i}^{\prime}\right\rangle\right|$.

An interior disjoint collection $\mathcal{P}$ of segment pairings on $C$ is full if $|\mathcal{P}|$ equals half the length of $C$. This means that the pairings in $\mathcal{P}$ cover $C$ up to a set of Lebesgue 1-dimensional measure zero.

A pairing of two segments which have an endpoint in common is called a fold and the common endpoint is called its folding point. The folding point in a fold is therefore not paired with any other point.

An interior disjoint collection $\mathcal{P}$ of segment pairings induces a reflexive and symmetric pairing relation, also denoted $\mathcal{P}$ :

$$
\mathcal{P}=\left\{\left(x, x^{\prime}\right): x, x^{\prime} \text { are paired or } x=x^{\prime}\right\} .
$$

Definitions 30 (Paper-folding scheme) A paper-folding scheme is a pair $(P, \mathcal{P})$ where $P \subset \mathbb{R}^{2}$ is a multipolygon with the intrinsic metric $d_{P}$ induced from $\mathbb{R}^{2}$, and $\mathcal{P}$ is a full interior disjoint collection of segment pairings on $\partial P$ (positively oriented). The metric quotient $S:=P / d_{P}^{\mathcal{P}}$ of $P$ under the semi-metric $d_{P}^{\mathcal{P}}$ induced by the pairing relation $\mathcal{P}$ is the associated paper space. When $S$ is a closed (compact without boundary) topological surface, then $(P, \mathcal{P})$ is called a surface paper-folding scheme and $S$ is the associated paper surface.

The projection map is denoted $\pi: P \rightarrow S$ and the quotient $G=\pi(\partial P) \subset S$ of the boundary is the scar. Notice that the restriction $\pi: \operatorname{Int}(P) \rightarrow S \backslash G$ is a homeomorphism.

The (quotient) metric on $S$ is denoted $d_{S}$. The metric $d_{G}$ on $G$ is defined to be the intrinsic metric as a subset of $S$ : it will be shown in Lemma 33 below that this is equal to the quotient metric on $G$ as a quotient of $\partial P$, where $\partial P$ is endowed with its intrinsic metric as a subset of $P$. 
The measure $\mathrm{m}_{G}$ on $G$ is defined to be the push-forward of Lebesgue 1-dimensional measure $\mathrm{m}_{\partial P}$ on $\partial P$. Hausdorff 1 -dimensional measure on $G$ is denoted $\mu_{G}^{1}-$ it will be shown in Lemma 33 that $\mu_{G}^{1}=\frac{1}{2} \mathrm{~m}_{G}$.

Example 35 below provides simple concrete examples of paper-folding schemes. The next definitions distinguish different types of points in a paper space.

Definitions 31 (Vertex, edge, singular point, planar point) For $k \in \mathbb{N} \cup\{\infty\}$, a point $x \in G$ is a vertex of valence $k$, or a $k$-vertex, if either

(i) $\# \pi^{-1}(x)=k \neq 2$; or

(ii) $\# \pi^{-1}(x)=k=2$ and $\pi^{-1}(x)$ contains a vertex of $P$.

Let $\mathcal{V}$ denote the set of all vertices of $G$.

The points of the paper space $S$ are divided into three types:

Singular points: vertices of valence $\infty$ and accumulations of vertices. Let $\mathcal{V}^{s}$ denote the set of singular points.

Regular vertices: vertices which are not singular.

Planar points: all other points of $S$ : that is, the points of $S \backslash \overline{\mathcal{V}}$.

The closures of the connected components of $G \backslash \overline{\mathcal{V}}$ are called edges of the scar $G$.

\section{Remarks 32}

(i) When discussing paper foldings, symbols with tildes will usually refer to objects in the source $P$ and symbols without tildes to objects in the quotient $S$. Thus, for example, $\tilde{x}$ may denote a point in $P$ and $x$ its projection in $S$; or $\left\langle\tilde{\alpha}, \widetilde{\alpha}^{\prime}\right\rangle$ may be a pairing on $\partial P$ and $\alpha:=\pi(\widetilde{\alpha})=\pi\left(\widetilde{\alpha}^{\prime}\right) \subset G$. For notational simplicity, however, tildes will be omitted when all objects being discussed lie in $P$.

(ii) The metric on a multipolygon $P$ is always the intrinsic metric induced by the metric on $\mathbb{R}^{2}$, denoted $d_{P}$. The symbol $d_{P}^{\mathcal{P}}$ will be used to denote both the semi-metric on $P$ induced by the pairing relation and the distance between $\sim_{\mathcal{P}}$ equivalence classes: if $\tilde{x}, \tilde{y} \in P$ and $[\tilde{x}],[\tilde{y}]$ denote their $\sim_{\mathcal{P}}$-classes, then

$$
d_{P}^{\mathcal{P}}([\tilde{x}],[\tilde{y}]):=d_{P}^{\mathcal{P}}(\tilde{x}, \tilde{y}) .
$$

Thus, if $x=\pi(\tilde{x}), y=\pi(\tilde{y}) \in S$, then $d_{S}(x, y):=d_{P}^{\mathcal{P}}([\tilde{x}],[\tilde{y}])$. 
(iii) The endpoints of paired segments need not necessarily project to vertices or singular points. For example, a segment pairing $\left\langle\alpha, \alpha^{\prime}\right\rangle$ can be split into two segment pairings $\left\langle\beta, \beta^{\prime}\right\rangle,\left\langle\gamma, \gamma^{\prime}\right\rangle$ by subdividing the segments $\alpha$ and $\alpha^{\prime}$, and the common endpoint of $\beta$ and $\gamma$ then projects to a planar point (see also Definition 39).

The next lemma summarises the properties of the metric and measure on the scar $G$ which will be important later. One of the issues which it addresses is the equivalence of the two natural ways to define a metric on $G$ : as the intrinsic metric induced by the inclusion of $G$ as a subset of $\left(S, d_{S}\right)$; and as the quotient metric coming from the intrinsic metric on $\partial P$.

Lemma 33 Let $G$ be the scar of the paper-folding scheme $(P, \mathcal{P})$. Then:

(a) The set of planar points is open and dense in the scar $G$, while the set $\overline{\mathcal{V}}$ of vertices and singular points is a closed nowhere dense subset of $G$ with zero $\mathrm{m}_{G}$-measure.

(b) The intrinsic metric on $G$ induced by the inclusion $G \subset S$ agrees with the quotient metric on $G=\partial P / d_{\partial P}^{\mathcal{P}}$ induced by the intrinsic metric $d_{\partial P}$ on $\partial P$.

(c) $G$ has Hausdorff dimension 1, and Hausdorff 1-dimensional measure $\mu_{G}^{1}$ on $G$ is equal to $\frac{1}{2} \mathrm{~m}_{G}$.

(d) Every arc $\gamma$ in $G$ is rectifiable, and $|\gamma|_{G}=\frac{1}{2} \mathrm{~m}_{G}(\gamma)$.

\section{Proof}

(a) The set of planar points is open in $G$ by definition (it is the set of points which are neither vertices nor accumulations of vertices), and is dense in $G$ since it contains the image under the continuous surjection $\pi$ of the (dense) set of points of $\partial P$ which belong to interior pairs. The set of vertices and singular points is the complement of the set of planar points, and is therefore closed and nowhere dense.

$\pi^{-1}(\overline{\mathcal{V}})$ is contained in the complement of the set of points belonging to interior pairs, which has zero $\mathrm{m}_{\partial P}$-measure, so that $\mathrm{m}_{G}(\overline{\mathcal{V}})=0$.

(b) Observe first that the intrinsic metrics $d_{P}$ on $P$ and $d_{\partial P}$ on $\partial P$ satisfy the condition $d_{\partial P}(\tilde{x}, \tilde{y}) \geq d_{P}(\tilde{x}, \tilde{y})$ for all $\tilde{x}, \tilde{y} \in \partial P$ (since any path in $\partial P$ is also a path in $P$ ), and that $d_{\partial P}(\tilde{x}, \tilde{y})=d_{P}(\tilde{x}, \tilde{y})=d_{\mathbb{R}^{2}}(\tilde{x}, \tilde{y})$ if $\tilde{x}$ and $\tilde{y}$ lie in the same side of $P$. 
Let $d_{G}^{i}$ denote the intrinsic metric on $G$ as a subset of $\left(S, d_{S}\right)$, and $d_{G}^{q}$ denote the metric on $G$ considered as the quotient metric space of $\left(\partial P, d_{\partial P}\right)$ under the relation $\mathcal{P}$. It will be shown that $d_{G}^{i} \leq d_{G}^{q}$ and $d_{G}^{q} \leq d_{G}^{i}$, which will establish the result.

To show $d_{G}^{i} \leq d_{G}^{q}$ : Let $x, y \in G$, and write $D=d_{G}^{q}(x, y)$. Let $\varepsilon>0$ be any positive number. A path $\gamma$ in $G$ from $x$ to $y$ with length $|\gamma|_{S}<D+\varepsilon$ will be constructed, which will establish the result since $d_{G}^{i}(x, y) \leq|\gamma|_{S}$.

Let $\tilde{x}, \tilde{y} \in \partial P$ with $\pi(\tilde{x})=x$ and $\pi(\tilde{y})=y$. By definition of $d_{G}^{q}$, there is a $\mathcal{P}$-chain $\left(\left(\tilde{p}_{i}, \tilde{q}_{i}\right)\right)_{i=0}^{k}$ from $\tilde{x}$ to $\tilde{y}$ in $\partial P$ with length

$$
\mathrm{L}^{\mathcal{P}}\left(\left(\tilde{p}_{i}, \tilde{q}_{i}\right)\right)=\sum_{i=0}^{k} d_{\partial P}\left(\tilde{p}_{i}, \tilde{q}_{i}\right)<D+\varepsilon .
$$

For each $i$, let $\tilde{\gamma}_{i}$ be a path from $\tilde{p}_{i}$ to $\tilde{q}_{i}$ in $\partial P$ of length $d_{\partial P}\left(\tilde{p}_{i}, \tilde{q}_{i}\right)$ (the metric $d_{\partial P}$ is strictly intrinsic by Theorem 15), and let $\gamma_{i}=\pi \circ \tilde{\gamma}_{i}$. Since $\tilde{q}_{i} \mathcal{P} \tilde{p}_{i+1}$ for each $i$, the concatenation of the paths $\gamma_{i}$ is a path $\gamma$ in $G$ from $x$ to $y$; and $\left|\gamma_{i}\right|_{S} \leq\left|\tilde{\gamma}_{i}\right|_{P} \leq\left|\tilde{\gamma}_{i}\right|_{\partial P}=d_{\partial P}\left(\tilde{p}_{i}, \tilde{q}_{i}\right)$ (the first inequality is from Remark 17, the second follows from $d_{P} \leq d_{\partial P}$, and the equality is by choice of the $\left.\tilde{\gamma}_{i}\right)$. Hence $|\gamma|_{S}<D+\varepsilon$ as required.

To show $d_{G}^{q} \leq d_{G}^{i}$ : Let $x, y \in G$, and write $D=d_{G}^{i}(x, y)$. Let $\varepsilon>0$ be any positive number. A $\mathcal{P}$-chain in $\partial P$ which connects points $\tilde{x}$ above $x$ and $\tilde{y}$ above $y$ will be constructed with length less than $D+\varepsilon$, which will establish the result since $d_{G}^{q}(x, y)$ is less than or equal to the length of such a chain. By definition of $d_{G}^{i}$ there is a path $\gamma:[0,1] \rightarrow G$ from $x$ to $y$ with $|\gamma|_{S}<D+\varepsilon$. That is,

$$
\sum_{i=0}^{k-1} d_{S}\left(\gamma\left(t_{i}\right), \gamma\left(t_{i+1}\right)\right)<D+\varepsilon
$$

for any partition $0=t_{0}<t_{1}<\cdots<t_{k}=1$.

Construct such a partition as follows. Let $\tilde{e}_{0}$ be a side of $P$ which contains a point $\tilde{x}_{0}$ with $\pi\left(\tilde{x}_{0}\right)=\gamma(0)=x$, and let $t_{1} \in[0,1]$ be the greatest parameter for which there is a point $\tilde{x}_{1} \in \tilde{e}_{0}$ with $\pi\left(\tilde{x}_{1}\right)=\gamma\left(t_{1}\right)$.

If $t_{1} \neq 1$, then there must be a side $\tilde{e}_{1} \neq \tilde{e}_{0}$ of $P$ containing either $\tilde{x}_{1}$ or a point identified with it. Let $t_{2} \in\left[t_{1}, 1\right]$ be the greatest parameter for which there is a point $\tilde{x}_{2} \in \tilde{e}_{1}$ with $\pi\left(\tilde{x}_{2}\right)=\gamma\left(t_{2}\right)$. If $t_{2} \neq 1$, then there is a side $\tilde{e}_{2}$ distinct from $\tilde{e}_{1}$ and $\tilde{e}_{0}$ containing $\tilde{x}_{2}$ or a point identified with it. Let $t_{3} \in\left[t_{2}, 1\right]$ be the greatest parameter for which there is a point $\tilde{x}_{3} \in \tilde{e}_{2}$ with $\pi\left(\tilde{x}_{3}\right)=\gamma\left(t_{3}\right)$. Since $P$ has only finitely many sides, this process terminates after a finite number of steps, yielding (after removing repeated parameters if necessary) a partition 
$0=t_{0}<t_{1}<\cdots<t_{k}=1$ with the property that, for each $i$, there are points $\tilde{p}_{i}$ and $\tilde{q}_{i}$ above $\gamma\left(t_{i}\right)$ and $\gamma\left(t_{i+1}\right)$ which lie on the same side of $P$. In particular, $d_{\partial P}\left(\tilde{p}_{i}, \tilde{q}_{i}\right)=d_{S}\left(\gamma\left(t_{i}\right), \gamma\left(t_{i+1}\right)\right)$.

The sequence $\left(\left(\tilde{p}_{i}, \tilde{q}_{i}\right)\right)_{i=0}^{k-1}$ is therefore a $\mathcal{P}$-chain in $\partial P$ with length

$$
\sum_{i=0}^{k-1} d_{\partial P}\left(\tilde{p}_{i}, \tilde{q}_{i}\right)=\sum_{i=0}^{k-1} d_{S}\left(\gamma\left(t_{i}\right), \gamma\left(t_{i+1}\right)\right)<D+\varepsilon
$$

as required.

(c) Because $\pi: \partial P \rightarrow G$ is distance non-increasing, it follows from Lemma 20 that

$$
\mu_{G}^{1}(\overline{\mathcal{V}}) \leq \mu_{G}^{1}(\pi(\bar{E})) \leq \mu_{\partial P}^{1}(\bar{E})=\mathrm{m}_{\partial P}(\bar{E})=0,
$$

where $E \subset \partial P$ is the set of endpoints of segments in segment pairings. Since $\mathrm{m}_{G}(\overline{\mathcal{V}})=0$ as well, it is enough to show that $\mu_{G}^{1}=\frac{1}{2} \mathrm{~m}_{G}$ on $G \backslash \overline{\mathcal{V}}$. However this set has countably many connected components, each an open arc which is the homeomorphic and locally isometric image under $\pi$ of exactly two disjoint open $\operatorname{arcs}$ in $\partial P$, and the result follows.

Since $\mu_{G}^{1}(G)>0$ is finite, $G$ has Hausdorff dimension 1 as required.

(d) Let $\gamma$ be an arc in $G$, consider a partition of $\gamma$ with points $x_{0}, \ldots, x_{n} \in \gamma$, and let $\gamma_{i}$ be the subarc of $\gamma$ with endpoints $x_{i}$ and $x_{i+1}$. Then, by Lemma 18,

$$
\sum_{i=0}^{n-1} d_{S}\left(x_{i}, x_{i+1}\right) \leq \sum_{i=0}^{n-1} \operatorname{diam}_{G}\left(\gamma_{i}\right) \leq \sum_{i=0}^{n-1} \mu_{G}^{1}\left(\gamma_{i}\right) \leq \mu_{G}^{1}(\gamma),
$$

so that $\gamma$ is rectifiable. That $|\gamma|_{G}=\frac{1}{2} \mathrm{~m}_{G}(\gamma)$ is immediate from (c) and Lemma 19.

The main focus of this article is on paper-folding schemes whose quotient is a surface and, in particular, a sphere. For clarity of exposition, attention will be concentrated on plain folding schemes: these are the simplest type of paper foldings, for which the paper space is always a sphere and the scar is always a dendrite (Theorem 42).

Definitions 34 (Unlinked pairing, plain paper-folding scheme) Let $\gamma$ be a polygonal arc or polygonal simple closed curve.

Two pairs of (not necessarily distinct) points $\left\{x, x^{\prime}\right\}$ and $\left\{y, y^{\prime}\right\}$ of $\gamma$ are unlinked if one pair is contained in the closure of a connected component of the complement of the other. Otherwise they are linked. 
A symmetric and reflexive relation $\mathrm{R}$ on $\gamma$ is unlinked if any two unrelated pairs of related points are unlinked: that is, if $x \mathrm{R} x^{\prime}, y \mathrm{R} y^{\prime}$, and neither $x$ nor $x^{\prime}$ is related to either $y$ or $y^{\prime}$, then $\left\{x, x^{\prime}\right\}$ and $\left\{y, y^{\prime}\right\}$ are unlinked.

An interior disjoint collection $\mathcal{P}$ of segment pairings on $\gamma$ is unlinked if the corresponding relation $\mathcal{P}$ is unlinked.

A paper-folding scheme $(P, \mathcal{P})$ is plain if $P$ is a single polygon and $\mathcal{P}$ is unlinked.

Example 35 Consider the unit square $P=\left\{(a, b) \in \mathbb{R}^{2}: 0 \leq a, b \leq 1\right\}$ and pick a decreasing sequence of real numbers $\left(a_{i}\right)_{i \in \mathbb{N}}$ such that $\sum a_{i}=1 / 2$. Define the following segment pairings on $\partial P$ (Figures 1 and 2). The two vertical sides are paired and the top side is folded in half. On the bottom side define countably many folds of length $a_{i}$ (that is, pairings of two segments with one endpoint in common, each of length $a_{i}$ ), with pairwise disjoint interiors.

In Figure 1 the folds are placed in order of decreasing length from right to left with coincident endpoints. The complement of the folds is the bottom left vertex of $P$ : all of the fold endpoints are identified with this vertex in the quotient space $S$.

In Figure 2, the folds are arranged in such a way that they are all disjoint. The closure of the complement of the folds is a Cantor set of Lebesgue measure 0 , uncountably many of whose points are unpaired. This Cantor set is identified to a point in the quotient space $S$.

In both examples (and for any other way of arranging the folds) the paper-folding scheme is unlinked, and hence the quotient space is a topological sphere by Theorem 42 below. Notice also that the scars corresponding to the two schemes are isometric $\infty$-ods (Definition 12).

These simple examples will be revisited in Example 43 below.

\section{The topological and metric structure of paper spaces}

The first main theorem of this section, Theorem 42, states that the paper space associated to a plain paper folding is a sphere, and its scar is a dendrite. The tools used to prove this result are the dendrite quotient theorem (Theorem 7), which gives sufficient conditions for a quotient of a continuum to be a dendrite; and Moore's theorem (Theorem 5), which gives sufficient conditions for a quotient of a sphere to be a sphere.

In Section 4.2 the topological structure of general paper spaces is considered: it is shown that they are paper surfaces provided that the boundary identifications are only finitely linked, and the structure of the scar in this case is described (Theorem 50). Finally, Section 4.3 contains a discussion of the metric structure of paper spaces. 

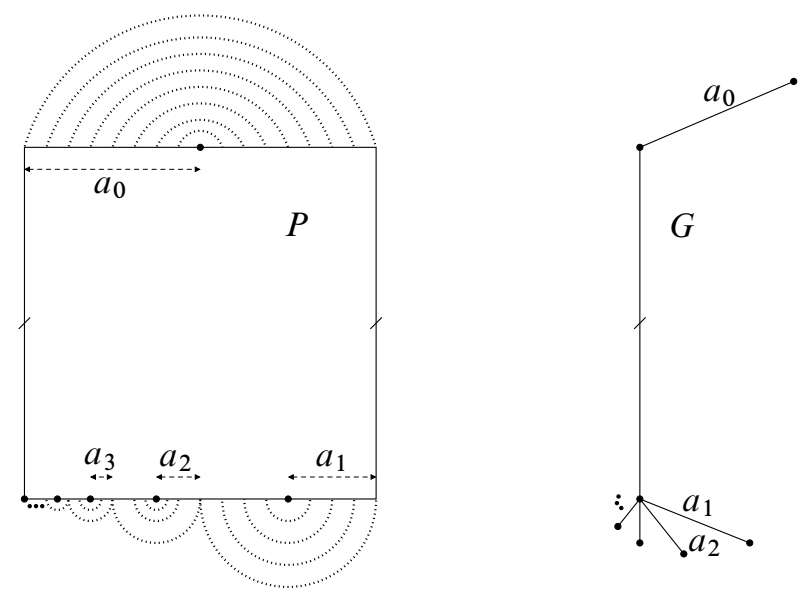

Figure 1: A paper-folding scheme and the associated scar
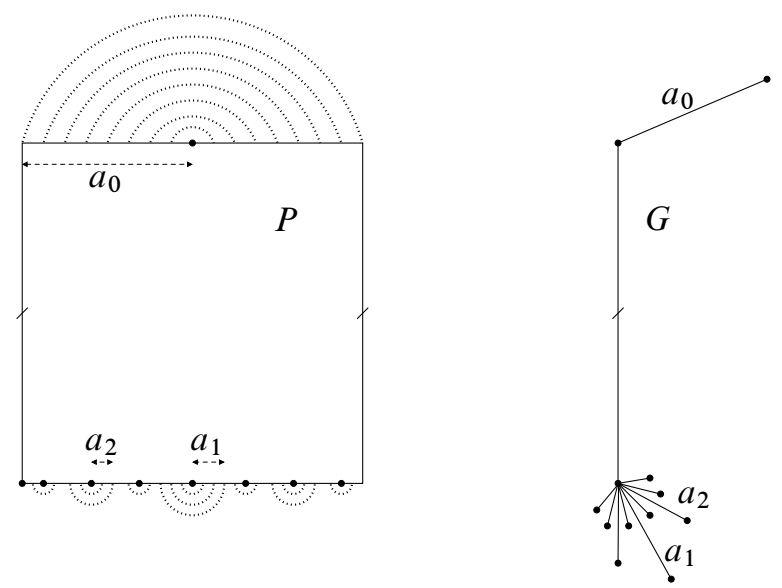

Figure 2: Another paper-folding scheme and the associated scar

\subsection{The plain paper folding structure theorem}

Many of the technical lemmas needed in the proof of the plain paper folding structure theorem are common to the general case and are not simplified by the plainness assumption. Thus in the beginning of this section $(P, \mathcal{P})$ is a general paper folding scheme: that is, $\mathcal{P}$ is a full interior disjoint collection of segment pairings on the boundary $\partial P$ of a multipolygon $P$; the equivalence relation induced by the semimetric $d_{P}^{\mathcal{P}}$ is denoted $\sim_{\mathcal{P}}$; the paper space is denoted $S$, the scar $G$, and the projection map $\pi: P \rightarrow S$; and if $x \in P$, its $\sim_{\mathcal{P}}$-equivalence class is denoted $[x]$. 
Definitions 36 (Plain arc, maximal plain arc) Let $(P, \mathcal{P})$ be a paper-folding scheme. An $\operatorname{arc} \gamma \subset \partial P$ is plain if:

(a) Every pairing in $\mathcal{P}$ which intersects the interior of $\gamma$ is contained in $\gamma$ (that is, if $\left\langle\alpha, \alpha^{\prime}\right\rangle$ is a segment pairing and either $\alpha$ or $\alpha^{\prime}$ intersects the interior of $\gamma$, then both $\alpha$ and $\alpha^{\prime}$ are contained in $\gamma$ ); and

(b) The restriction of $\mathcal{P}$ to $\gamma$ is unlinked (Definitions 34).

A component $\gamma$ of $\partial P$ is plain if it is $\mathcal{P}$-saturated and the restriction of $\mathcal{P}$ to $\gamma$ is unlinked.

A plain arc is maximal if it is not strictly contained in any plain arc or component.

\section{Remarks 37}

(a) Recall (Definitions 34) that a paper folding scheme $(P, \mathcal{P})$ is itself called plain if $P$ is a single polygon and $\mathcal{P}$ is unlinked. With the definitions just introduced this is equivalent to saying that $P$ consists of a single polygon whose boundary $\partial P$ is plain as just defined.

(b) Every plain arc contains at least one pairing: pairings are dense in $\partial P$ and so intersect the interior of — and thus must be contained in — any plain arc or component.

(c) If every pairing which intersects the interior of $\gamma$ is contained in $\gamma$, then the same holds for the complement of $\gamma$ in $\partial P$ : every pairing which intersects $\partial P \backslash \gamma$ is contained in $\overline{\partial P \backslash \gamma}$.

(d) The union of two plain arcs with non-empty intersection is plain. Hence any two distinct maximal plain arcs are disjoint.

Lemma 38 If $\gamma$ is a plain arc, then its endpoints are $\sim_{\mathcal{P}}$-equivalent.

Proof Let $a$ and $b$ be the endpoints of $\gamma$ : for convenience, they will be referred to as the left and right endpoints respectively, and the points of $\gamma$ will be ordered from $a$ to $b$.

Let $\varepsilon>0$ be any positive number. It will be shown that $d_{P}^{\mathcal{P}}(a, b)<\varepsilon$, which will establish the result.

By fullness, there is a finite collection of segment pairings $\left\{\left\langle\alpha_{r}, \alpha_{r}^{\prime}\right\rangle\right\}_{r=1}^{N}$ contained in $\gamma$ which cover $\gamma$ to within length $\varepsilon$. Label the segments so that $\alpha_{r}$ is to the left of $\alpha_{r}^{\prime}$ for each $r$. 
Let $p_{0}=a$, and construct a $\mathcal{P}$-chain from $a$ to $b$ inductively as follows: for each $i \geq 0$, let $q_{i}$ be the leftmost point of $\left[p_{i}, b\right]$ which is a left hand endpoint of some $\alpha_{r(i)}$, and let $p_{i+1}$ be the right hand endpoint of $\alpha_{r(i)}^{\prime}$, so that $q_{i} \mathcal{P} p_{i+1}$. Since the subcollection of segment pairings is finite, there is some $k$ such that $\left[p_{k}, b\right]$ is disjoint from all of the $\alpha_{r}$ : set $q_{k}=b$. By construction, $p_{0} \leq q_{0}<p_{1} \leq q_{1}<\cdots<p_{k} \leq q_{k}$.

The intervals $\left(p_{i}, q_{i}\right)$ are disjoint from all of the segments $\alpha_{r}$ by choice of $q_{i}$. They are also disjoint from the $\alpha_{r}^{\prime}$. For if $\left(p_{i}, q_{i}\right)$ were to contain some $\alpha_{r}^{\prime}$, then $\alpha_{r}$ couldn't lie in any $\left(p_{j}, q_{j}\right)$ with $j<i$ as above; and if $\alpha_{r}$ were to lie in $\left(q_{j}, p_{j+1}\right)$ then the segments $\alpha_{r(j)}<\alpha_{r}<\alpha_{r(j)}^{\prime}<\alpha_{r}^{\prime}$ would be linked, contradicting plainness.

Hence

$$
d_{P}^{\mathcal{P}}(a, b) \leq \mathrm{L}^{\mathcal{P}}\left(\left(p_{i}, q_{i}\right)\right)=\sum_{i=0}^{k} d\left(p_{i}, q_{i}\right)<\varepsilon
$$

as required.

Definition 39 (Subpairing) Let $\left\langle\alpha, \alpha^{\prime}\right\rangle$ be a pairing in $\mathcal{P}, \beta$ be a subarc of $\alpha$, and $\beta^{\prime}$ be the corresponding subarc of $\alpha^{\prime}$. Then $\left\langle\beta, \beta^{\prime}\right\rangle$ is said to be a subpairing of $\left\langle\alpha, \alpha^{\prime}\right\rangle$.

Lemma 40 Let $\gamma$ be a plain arc or component of $\partial P$, and $\left\langle\alpha, \alpha^{\prime}\right\rangle$ be a pairing in $\mathcal{P}$, or a subpairing of a pairing in $\mathcal{P}$, which is contained in $\gamma$. Let $z \in \gamma \backslash\left(\stackrel{\circ}{\alpha} \cup \stackrel{\circ}{\alpha}^{\prime}\right)$, and if $\gamma$ is an arc then assume that $\stackrel{\circ}{\alpha} \cup \stackrel{\circ}{\alpha}$ separates $z$ from the endpoints of $\gamma$.

Then the $\mathcal{P}$-semi-distance along $\partial P$ from $z$ to any point $w$ in a different component of $\partial P \backslash(\stackrel{\circ}{\alpha} \cup \stackrel{\circ}{\alpha})$ is bounded below by the length $L$ of $\left\langle\alpha, \alpha^{\prime}\right\rangle$ :

$$
d_{\partial P}^{\mathcal{P}}(z, w) \geq L:=\left|\left\langle\alpha, \alpha^{\prime}\right\rangle\right|
$$

Proof Let $\gamma_{1}$ be the component of $\partial P \backslash(\stackrel{\circ}{\alpha} \cup \stackrel{\circ}{\alpha})$ which contains $z$, and let $\gamma_{2}$ denote $\partial P \backslash\left(\gamma_{1} \cup \stackrel{\alpha}{\alpha} \cup \stackrel{\circ}{\alpha}^{\prime}\right)$. Since all of the pairings in $\mathcal{P}$ are unlinked with $\left\langle\alpha, \alpha^{\prime}\right\rangle$, both $\gamma_{1}$ and $\gamma_{2}$ are $\mathcal{P}$-saturated.

Let $\left(\left(p_{i}, q_{i}\right)\right)_{i=0}^{k}$ be a $\mathcal{P}$-chain in $\partial P$ from $z$ to $w$. By saturation of $\gamma_{1}$, if $q_{r} \in \gamma_{1}$ then $p_{r+1} \in \gamma_{1}$ : hence the last point of the chain which lies in $\gamma_{1}$ must be some $p_{r}$; and by saturation of $\gamma_{2}$, the first point of the chain after $p_{r}$ which lies in $\gamma_{2}$ must be some $q_{s}$ with $s \geq r$.

If $s=r$ then $d_{\partial P}\left(p_{r}, q_{r}\right) \geq L$. If $s>r$, then the points $q_{r}, p_{r+1}, q_{r+1}, \ldots, p_{s}$ all lie in $\stackrel{\circ}{\alpha} \cup \stackrel{\circ}{\alpha}^{\prime}$. Let $\xi, \xi^{\prime}:[0, L] \rightarrow \partial P$ be parametrisations of $\alpha$ and $\alpha^{\prime}$ by arc length, and 
let $t_{i}$ be parameters such that $q_{i}$ and $p_{i+1}$ are equal to $\xi\left(t_{i}\right)$ or $\xi^{\prime}\left(t_{i}\right)$ for $r \leq i<s$. Then

$$
d_{\partial P}^{\mathcal{P}}(z, w) \geq \sum_{i=r}^{s} d_{\partial P}\left(p_{r}, q_{r}\right) \geq t_{r}+\sum_{i=r+1}^{s-1}\left|t_{i}-t_{i-1}\right|+\left|L-t_{s-1}\right| \geq L
$$

as required.

Recall that an arc $\gamma$ is plain if the pairing relation $\mathcal{P}$ has the properties that: every pairing in $\mathcal{P}$ which intersects $\stackrel{\gamma}{\gamma}$ is contained in $\gamma$; and the restriction of $\mathcal{P}$ to $\gamma$ is unlinked. The following lemma states that analogous properties also hold for the equivalence relation $\sim_{\mathcal{P}}$.

Lemma 41 Let $\gamma$ be a plain arc and let $a, b$ denote its endpoints. Then $\sim_{\mathcal{P}}$ is unlinked on $\gamma$ and $\gamma \backslash[a]=\gamma \backslash[b]$ is $\sim_{\mathcal{P}}$-saturated. Similarly, if $\gamma$ is a plain component of $\partial P$, then $\sim_{\mathcal{P}}$ is unlinked on $\gamma$ and $\gamma$ is $\sim_{\mathcal{P}}$-saturated.

Proof Unlinkedness: Assume that $\gamma$ is a plain arc: the case where it is a plain component of $\partial P$ is analogous. Let $x, x^{\prime}, y$, and $y^{\prime}$ be points of $\gamma$ with $x \sim_{\mathcal{P}} x^{\prime}$ and $y \sim_{\mathcal{P}} y^{\prime}$, such that the pairs $\left\{x, x^{\prime}\right\}$ and $\left\{y, y^{\prime}\right\}$ are linked (and in particular all four points are distinct). It is necessary to show that all four points are $\sim_{\mathcal{P}}$ equivalent.

Without loss of generality, suppose that $a \leq x<y<x^{\prime}<y^{\prime} \leq b$ with respect to an order on $\gamma$. The three arcs $[x, y],\left[y, x^{\prime}\right]$, and $\left[x^{\prime}, y^{\prime}\right]$ are all plain, by Lemma 40: for example, if a point of $(x, y)$ were paired with a point of $[a, x)$ or a point of $\left(x^{\prime}, b\right]$, the segment pairing realising this would separate $x$ from $x^{\prime}$, contradicting $x \sim_{\mathcal{P}} x^{\prime}$; while if it were paired with a point of $\left(y, x^{\prime}\right]$ the segment pairing would separate $y$ from $y^{\prime}$. Hence $x \sim_{\mathcal{P}} y \sim_{\mathcal{P}} x^{\prime} \sim_{\mathcal{P}} y^{\prime}$ by Lemma 38 .

Saturation: Let $\gamma$ be a plain arc, and $x \in \stackrel{\circ}{\gamma}$. It is required to show that either $x \sim_{\mathcal{P}} a$, or $[x] \subset \gamma$.

If there is a pairing or subpairing in $\gamma$ which separates $x$ from $a$ and $b$, then it separates $x$ from $\partial P \backslash \gamma$, and $[x] \subset \gamma$ by Lemma 40 .

If there is no such pairing, then the $\operatorname{arcs}[a, x]$ and $[x, b]$ are both plain, and $[x]=[a]=[b]$ by Lemma 38 .

If $\gamma$ is a plain component of $\partial P$ then it is clear that no point of $\gamma$ can be $\sim_{\mathcal{P}}$-equivalent to a point in another boundary component of $P$.

Theorem 42 (Topological structure of a plain paper folding) The quotient $S$ of a plain paper-folding scheme is a topological sphere, and its scar $G$ is a dendrite. 
Proof Since both $\partial P$ and $P$ are compact, the scar $G$ and the paper space $S$ are homeomorphic to the topological quotients $\partial P / \sim_{\mathcal{P}}$ and $P / \sim_{\mathcal{P}}$ respectively by Theorem 13 .

To show that $G$ is a dendrite, it is enough by Theorem 7 to show that the decomposition $\mathcal{G}$ of $\partial P$ into equivalence classes of $\sim_{\mathcal{P}}$ is dendritic. That $\mathcal{G}$ is non-separated is immediate by Lemma 41: saying that $\sim_{\mathcal{P}}$ is unlinked is the same as saying that $\mathcal{G}$ is non-separated. Thus it remains to show that if $[x] \in \mathcal{G}$ and $y \notin[x]$, then there is some $[z] \in \mathcal{G}$ which separates $y$ and $[x]$.

Let $A \subset \partial P$ be the maximal arc containing $y$ which is disjoint from $[x]$. Thus $A$ is an open arc with endpoints $x_{1}, x_{2} \in[x]$ (possibly $x_{1}=x_{2}$ if $[x]$ is a single point). Since $\sim_{\mathcal{P}}$ is unlinked, $A$ is $\sim_{\mathcal{P}}$-saturated.

If there are paired points $z_{1}$ and $z_{2}$ in different components of $A \backslash\{y\}$, pick nearby interior paired points $z_{1}^{\prime}$ and $z_{2}^{\prime}$, so that $\left[z_{1}^{\prime}\right]=\left[z_{2}^{\prime}\right]$ does not contain $y$. Then $\left[z_{1}^{\prime}\right]$ separates $y$ and $[x]$ as required.

If there are no such points then $\left[x_{1}, y\right]$ and $\left[y, x_{2}\right]$ are plain, so that $y \in[x]$ by Lemma 38, a contradiction.

To show that the paper space $S$ is a sphere, it is convenient to change coordinates by a suitable homeomorphism so that $\partial P$ is the unit circle $S^{1}$ in $\widehat{\mathbb{C}}$, and $P$ is the exterior of the unit disk $\mathbb{D} \subset \widehat{\mathbb{C}}$ : the unit disk $\mathbb{D}$ will be regarded as the hyperbolic plane. This change of coordinates is purely for convenience: the constructions don't use hyperbolic geometry in any essential way.

Connect the points $x, y$ of each interior pair in $\partial P$ with the geodesic having them as endpoints. Because $\mathcal{P}$ is unlinked, these geodesics are pairwise disjoint. Let $\mathcal{L}$ be the geodesic lamination obtained by taking the closure of the union of such geodesics. Because the equivalence relation $\sim_{\mathcal{P}}$ is closed, every geodesic in $\mathcal{L}$ joins a pair of equivalent points.

If $U$ is a component of $\mathbb{D} \backslash \mathcal{L}$, let $\bar{U}$ denote its closure in $S$. Then $\bar{U}$ is a closed disk, whose boundary is the union of some geodesics (including endpoints) in $\mathcal{L}$ joining equivalent points which are not in interior pairs, and other points of $\partial P$.

Let $x, y \in \partial P$ be distinct points which are not in interior pairs, and let $[x, y] \subset \partial P$ be an interval with these endpoints. Then the following conditions are equivalent:

(i) $x \sim \mathcal{P} y$.

(ii) $[x, y]$ is plain.

(iii) $x$ and $y$ lie in the closure $\bar{U}$ of the same component $U$ of $\mathbb{D} \backslash \mathcal{L}$ or are the endpoints of a geodesic in $\mathcal{L}$. 
For (i) $\Longleftrightarrow$ (ii), suppose that $[x, y]$ is not plain. Since $\mathcal{P}$ is unlinked, this means that there is a pairing $\left\langle\alpha, \alpha^{\prime}\right\rangle$ which intersects $(x, y)$ but is not contained in $[x, y]$. The assumption that $x$ and $y$ are not in interior pairs means that one of the segments, say $\alpha$, is contained in $[x, y]$ while the other, $\alpha^{\prime}$, is disjoint from $(x, y)$. Thus $x$ and $y$ lie in different components of $\partial P \backslash\left(\stackrel{\circ}{\alpha} \cup \stackrel{\circ}{\alpha^{\prime}}\right)$, so that $x \chi_{\mathcal{P}} y$ by Lemma 40 . The converse is immediate from Lemma 38.

For (ii) $\Longleftrightarrow$ (iii): Suppose that $[x, y]$ is plain and that the geodesic with endpoints $x$ and $y$ is not in $\mathcal{L}$. Then there are no geodesics in $\mathcal{L}$ with one endpoint in $(x, y)$ and the other disjoint from $[x, y]$, and hence the geodesic with endpoints $x$ and $y$ lies in a single component $U$ of $\mathbb{D} \backslash \mathcal{L}$, so that $x, y \in \bar{U}$. If $[x, y]$ is not plain then as above there is a pairing $\left\langle\alpha, \alpha^{\prime}\right\rangle$ with $\stackrel{\circ}{\alpha} \subset(x, y)$ and $\stackrel{\circ}{\alpha}^{\prime} \cap[x, y]=\varnothing$, and any geodesic connecting paired points in $\stackrel{\circ}{\alpha}$ and $\stackrel{\circ}{\alpha}^{\prime}$, together with its endpoints, separates $x$ from $y$ in $\overline{\mathbb{D}}$.

Therefore two distinct points $x, y \in \partial P$ satisfy $x \sim_{\mathcal{P}} y$ if and only if either they are the endpoints of a geodesic in $\mathcal{L}$, or they lie in the closure of the same component of $\mathbb{D} \backslash \mathcal{L}$, so that a decomposition $\mathcal{G}$ of $\widehat{\mathbb{C}}$ which realises $\sim_{\mathcal{P}}$ can be constructed with the following elements:

(I) Closures $\bar{U}$ of components of $\mathbb{D} \backslash \mathcal{L}$;

(II) Geodesics in $\mathcal{L}$, together with endpoints, which are not contained in elements of type (I); and

(III) Points which are not contained in elements of types (I) and (II).

The elements of $\mathcal{G}$ are closed disks, arcs, and points, and so are connected and do not separate $\widehat{\mathbb{C}}$. To complete the proof using Moore's theorem, it is therefore only required to show that $\mathcal{G}$ is upper semi-continuous. So let $x_{n} \rightarrow x$ and $y_{n} \rightarrow y$ be sequences in $\widehat{\mathbb{C}}$ such that $x_{n}$ and $y_{n}$ belong to the same decomposition element for all $n$ : it is required to show that $x$ and $y$ belong to the same decomposition element.

If $x_{n}$ and $y_{n}$ belong to elements of type (III) for infinitely many $n$, then $x_{n}=y_{n}$ for these values of $n$, and hence $x=y$. Passing to a subsequence, it can therefore be assumed that none of the $x_{n}$ or $y_{n}$ belong to an element of type (III).

Suppose that infinitely many of the $x_{n}$ and $y_{n}$ belong to geodesics $\gamma_{n}$ of type (II). If there is no positive lower bound on the distance in $\partial P$ between the endpoints of the $\gamma_{n}$ then $x=y$. If there is such a lower bound then the geodesics $\gamma_{n}$ converge to a geodesic (together with endpoints) $\gamma$ in $\mathcal{L}$ which contains $x$ and $y$.

It can therefore be assumed that all of the $x_{n}$ and $y_{n}$ belong to elements $\bar{U}_{n}$ of type (I). For each $n$, let $\alpha_{n} \subset \bar{U}_{n}$ be the geodesic arc with endpoints $x_{n}$ and $y_{n}$, and let $\gamma_{n}$ be the 
geodesic containing $\alpha_{n}$. If there is no lower bound on the distance in $\partial P$ between the endpoints of the $\gamma_{n}$, then $x=y$. If there is such a lower bound then the geodesics $\gamma_{n}$ converge to a geodesic $\gamma$ containing $x$ and $y$.

If either $x=y$ or $\gamma$ is a geodesic in $\mathcal{L}$, then $x$ and $y$ lie in the same decomposition element. Otherwise, the geodesic arc $\alpha \subset \gamma$ connecting $x$ and $y$ is disjoint from $\mathcal{L}$ (if it intersected a geodesic in $\mathcal{L}$, then so would $\alpha_{n}$ for large $n$ ), and hence $x$ and $y$ lie in the same component of $\mathbb{D} \backslash \mathcal{L}$.

Example 43 Here the construction in the above proof is considered for the paperfolding schemes of Example 35. Recall that $P$ is a square, the top side of $P$ is folded in half, the vertical sides are paired, and folds of lengths $a_{i}$ are placed along the bottom side, either continuously from right to left (Figure 1) or disjointly (Figure 2).

Figure 3 depicts the decomposition of $\widehat{\mathbb{C}}$ which realises $\sim_{\mathcal{P}}$ in the scheme of Figure 1. There is a single decomposition element of type (I) which is denoted $g_{\infty}$ : it includes countably many points of $\partial P$ (the fold endpoints and the bottom left corner of $P$ ), and countably many geodesics joining these points in pairs. The other geodesics (including their endpoints on $\partial P$ ) are decomposition elements of type (II), and the points of $\operatorname{Int}(P)$ are decomposition elements of type (III).

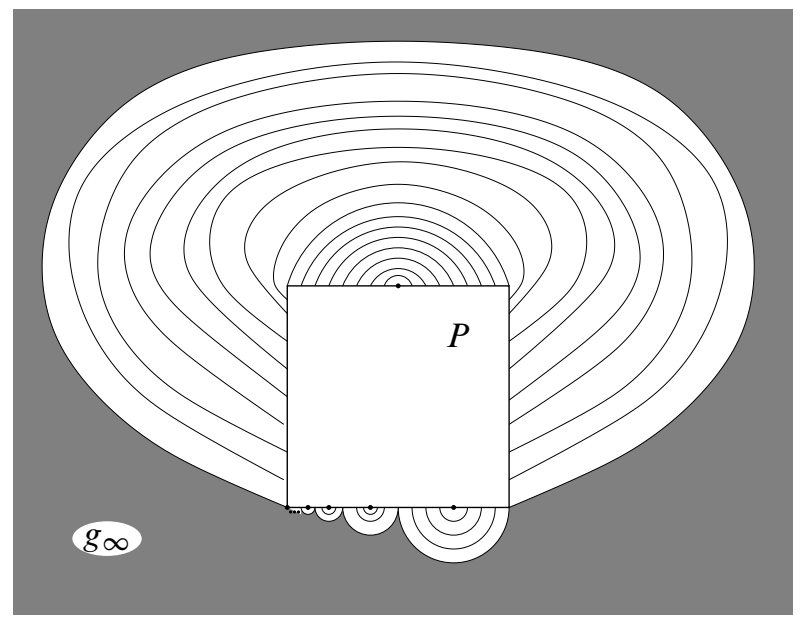

Figure 3: The musc decomposition realising $\sim_{\mathcal{P}}$ in the paper-folding scheme of Figure 1

The decomposition for the scheme of Figure 2 is similar: in this case the single decomposition element $g_{\infty}$ of type (I) intersects $\partial P$ in a Cantor set, only countably many of whose points are connected by geodesics. 


\subsection{Surface paper folding schemes}

In this section the topological structure of the paper space $S$ of a general paper-folding scheme $(P, \mathcal{P})$ is considered. It is clear that linked identifications around $\partial P$ create handles in the paper space, and that if there are infinitely many such handles then $S$ cannot be a compact surface. The main result of this section, Theorem 50, states that this is the only obstruction to $S$ being a paper surface, and describes the scar $G$ in the surface case: each component of $G$ (corresponding to a component of $S$ ) is the union of countably many dendrites (one corresponding to each maximal plain arc in $\partial P$ ) and a finite connected graph (corresponding to the linked identifications), each dendrite being attached to the connected graph at a single point.

The first step is to reduce to the case in which $P$ is a single polygon: or, more accurately, a single closed disk, since the polygonal structure of $P$ will be lost in the constructions which follow.

Let $\Upsilon$ be the finite graph whose vertices are the components $P_{i}$ of $P$, and which has an edge joining $P_{i}$ and $P_{j}$ if and only if there is a pairing $\left\langle\alpha, \alpha^{\prime}\right\rangle \in \mathcal{P}$ with $\alpha \subset \partial P_{i}$ and $\alpha^{\prime} \subset \partial P_{j}$. Then the connected components of $S$ correspond bijectively to the connected components of $\Upsilon$. In the remainder of the section it will be assumed that $\Upsilon$ is connected (if not, apply the results to each connected component in turn).

Let $T$ be a spanning subtree of $\Upsilon$. Pick one pairing corresponding to each edge of $T$, and realise the associated identifications. The resulting space is a topological disk $P^{\prime}$, and the remaining pairings induce a full interior disjoint collection $\mathcal{P}^{\prime}$ of arc pairings along $\partial P^{\prime}$. It therefore suffices to consider $\left(P^{\prime}, \mathcal{P}^{\prime}\right)$. As mentioned above, $P^{\prime}$ is not a polygon, but the polygonal structure is irrelevant to the topological structure of the quotient: the word "segment" will be understood to mean "arc", and the length of an arc in $\partial P^{\prime}$ is given by the minimal length of a subset of $\partial P$ which projects to it.

In the remainder of this section, then, it will be assumed that $P$ is a topological disk with rectifiable boundary, and that $\mathcal{P}$ is a full interior disjoint collection of arc pairings on $\partial P$. It will also be assumed that $(P, \mathcal{P})$ is not plain, since otherwise the results of Section 4.1 apply.

Let $\Pi=\left\{\gamma_{i}: i \in I\right\}$ be the set of maximal plain arcs in $\partial P$, where $I$ is a finite or countable index set. These arcs are mutually disjoint (Remarks 37(d)), and each component of $\partial P \backslash \bigcup_{i \in I} \gamma_{i}$ is an open arc (between any two elements of $\Pi$ lies an $\operatorname{arc} \alpha$ of a pairing of $\mathcal{P}$ whose interior is not contained in any plain arc).

Let $\mathcal{C}$ be the simple closed curve obtained from $\partial P$ by collapsing each element of $\Pi$ to a point (in fact the maximal plain arcs will not be collapsed: rather the identifications 
on them will be realised, and in particular their endpoints will be identified). The pairings in $\mathcal{P}$ which are not contained in any element of $\Pi$ induce a full interior disjoint collection $\mathcal{Q}$ of arc pairings on $\mathcal{C}$, with respect to which $\mathcal{C}$ contains no plain arcs, since the preimage in $\partial P$ of such a plain arc would be a plain arc intersecting the complement of the $\gamma_{i}$.

The aim now is to decompose $\mathcal{C}$ into maximal unlinked arcs.

Definitions 44 (Unlinked arc, maximal unlinked $\operatorname{arc}$ ) An $\operatorname{arc} \gamma$ in $\mathcal{C}$ is unlinked if

(a) Every $\operatorname{arc} \beta_{i}$ in a pairing in $\mathcal{Q}$ which intersects $\gamma^{\circ}$ is contained in $\gamma$;

(b) $\overline{\bigcup_{i} \beta_{i}^{\prime}}$ is an arc $\gamma^{\prime}$, where the $\beta_{i}^{\prime}$ are the arcs paired with the $\operatorname{arcs} \beta_{i}$ of (a); and

(c) The restriction of $\mathcal{Q}$ to $\gamma \cup \gamma^{\prime}$ is unlinked on $\mathcal{C}$.

The arc $\gamma^{\prime}$ is said to be paired with $\gamma$. An unlinked $\operatorname{arc} \gamma$ is maximal if it is not strictly contained in any other unlinked arc.

\section{Remarks 45}

(a) An unlinked arc $\gamma$ is disjoint from its paired $\operatorname{arc} \gamma^{\prime}$, as otherwise $\gamma \cup \gamma^{\prime}$ would be a plain arc.

(b) Let $\gamma$ be an unlinked arc with pair $\gamma^{\prime}$. Then the restriction of $\sim_{\mathcal{Q}}$ to $\gamma \cup \gamma^{\prime}$ is the arc pairing $\left\langle\gamma, \gamma^{\prime}\right\rangle$.

Lemma $46 \mathcal{C}$ can be written uniquely as a union of maximal unlinked arcs and points which are not contained in any unlinked arc. The maximal unlinked arcs intersect only at their endpoints.

Proof If $\gamma_{1}$ and $\gamma_{2}$ are unlinked arcs whose interiors intersect, then $\gamma_{1} \cup \gamma_{2}$ is also an unlinked arc. In particular, two distinct maximal unlinked arcs can only intersect at their endpoints.

Let $\beta$ be an arc in a pairing in $\mathcal{Q}$. Then $\beta$ is itself an unlinked arc. Let $\gamma_{\beta}$ be the union of all unlinked arcs containing $\beta$. Then $\gamma_{\beta}$ is either an arc, or an arc without one or both of its endpoints, which satisfies the conditions of Definitions 44 except for the possible absence of endpoints of $\gamma_{\beta}$ and $\gamma_{\beta}^{\prime}$. However, this means that the conditions also hold for $\gamma_{\beta}$ together with its endpoints, so that $\gamma_{\beta}$ is in fact an arc.

Hence every arc in a pairing in $\mathcal{Q}$ is contained in a unique maximal unlinked arc, and these arcs intersect only at their endpoints. If $x$ is a point of $\mathcal{C}$ which is not contained in any of these arcs, then any arc containing $x$ must intersect a paired $\operatorname{arc} \beta$ and hence the maximal arc $\gamma_{\beta}$ : therefore $x$ is not contained in any unlinked arc. 
Definition 47 (Finitely linked) $(P, \mathcal{P})$ is said to be finitely linked if either it is plain, or there are only finitely many maximal unlinked arcs in the decomposition of Lemma 46.

Remark 48 If $(P, \mathcal{P})$ is finitely linked, then there cannot be any points of $\mathcal{C}$ which are disjoint from all of the maximal unlinked arcs: that is, $\mathcal{C}$ can be written uniquely as a union of finitely many maximal unlinked arcs.

The following lemma is the main tool used in the proof of the converse of Theorem 50 below, that if $(P, \mathcal{P})$ is not finitely linked then $S$ is not a compact surface. The setting of the lemma is slightly different from that of the remainder of the paper $-P$ is a compact surface with a single boundary component $\mathcal{C}$ rather than a topological disk - but the concepts of arc pairings on $\mathcal{C}$, plain arcs, and finitely linked are defined analogously.

Lemma 49 Let $P$ be a compact surface with a single boundary component $\mathcal{C}$, and $\mathcal{P}$ be a full interior disjoint collection of arc pairings on $\mathcal{C}$ which is such that $\mathcal{C}$ contains no plain arcs, and is not finitely linked. Then there are maximal unlinked $\operatorname{arcs} \alpha$ and $\beta$, with paired $\operatorname{arcs} \alpha^{\prime}$ and $\beta^{\prime}$, such that $\left\langle\alpha, \alpha^{\prime}\right\rangle$ and $\left\langle\beta, \beta^{\prime}\right\rangle$ are linked; and carrying out the identifications corresponding to these arc pairings yields a compact surface $\hat{P}$ having a single boundary component $\hat{\mathcal{C}}$, with the properties that:

- the genus of $\widehat{P}$ is one greater than that of $P$;

- the induced arc pairings $\hat{\mathcal{P}}$ on $\hat{\mathcal{C}}$ are such that $\hat{\mathcal{C}}$ contains no plain arcs and $\hat{\mathcal{P}}$ is not finitely linked.

Proof First some notation will be introduced. Let $\mathcal{A}$ be the set of maximal unlinked arcs on $\mathcal{C}$, and $\phi: \mathcal{A} \rightarrow \mathcal{A}$ be the involution which takes each maximal unlinked arc to its pair. Fix an orientation of $\mathcal{C}$, and for each pair of distinct points $x, y \in \mathcal{C}$, write $[x, y]$ for the arc in $\mathcal{C}$ with initial and final endpoints $x$ and $y$.

A segment in $\mathcal{A}$ is a set of the form

$$
\llbracket x, y \rrbracket:=\{\gamma \in \mathcal{A}: \gamma \subset[x, y]\},
$$

where $x$ and $y$ are distinct points of $\mathcal{C}$ which do not lie in the interior of any element of $\mathcal{A}$. A segment is non-trivial if it has more than one element. Notice that each segment is endowed with a total order $<$ induced by the orientation on $\mathcal{C}$. Similarly, given distinct elements $\gamma$ and $\delta$ of $\mathcal{A}$, write $\llbracket \gamma, \delta \rrbracket$ for the segment $\llbracket x, y \rrbracket$, where $x$ is the initial point of $\gamma$ and $y$ is the final point of $\delta$, provided that these points do not coincide; and write $[\gamma, \delta]$ for the $\operatorname{arc}[x, y]$ in $\mathcal{C}$.

The set $\mathcal{A}$ has the following properties: 
(i) (The elements of $\mathcal{A}$ are maximal.) There are no two non-trivial disjoint segments $\llbracket x, y \rrbracket$ and $\llbracket x^{\prime}, y^{\prime} \rrbracket$ with the properties that: $\gamma \in \llbracket x, y \rrbracket$ if and only if $\phi(\gamma) \in$ $\llbracket x^{\prime}, y^{\prime} \rrbracket$; and $\gamma<\delta$ in $\llbracket x, y \rrbracket$ if and only if $\phi(\gamma)>\phi(\delta)$ in $\llbracket x^{\prime}, y^{\prime} \rrbracket$. (If there were such segments, then arcs in each segment could be coalesced to give larger unlinked arcs.)

(ii) (There are no plain arcs.) Let $\gamma \in \mathcal{A}$, and suppose that $\phi(\delta) \in \llbracket \gamma, \phi(\gamma) \rrbracket$ whenever $\delta \in \llbracket \gamma, \phi(\gamma) \rrbracket$. Then there are $\alpha, \beta \in \llbracket \gamma, \phi(\gamma) \rrbracket$ such that $\alpha<\beta<\phi(\alpha)<\phi(\beta)$ with respect to the order on $\llbracket \gamma, \phi(\gamma) \rrbracket$. (Otherwise $[\gamma, \phi(\gamma)]$ would be a plain arc.)

The existence of the maximal unlinked arcs $\alpha$ and $\beta$ in the statement of the lemma is immediate from property (ii). Let $\gamma$ be any element of $\mathcal{A}$. If there is some $\delta \in \mathcal{A}$ with $\delta \in \llbracket \gamma, \phi(\gamma) \rrbracket$ but $\phi(\delta) \notin \llbracket \gamma, \phi(\gamma) \rrbracket$, then set $\alpha=\gamma$ and $\beta=\delta$. If there is no such $\delta$, then suitable arcs $\alpha$ and $\beta$ exist by property (ii).

It will be assumed in the remainder of the proof that the $\operatorname{arcs} \alpha, \alpha^{\prime}, \beta$, and $\beta^{\prime}$ have no common endpoints: only minor modifications are required in the case where they share some endpoints.

Carrying out the identifications corresponding to these arcs clearly yields a compact surface $\widehat{P}$ with genus one greater than that of $P$, having a single boundary component $\widehat{\mathcal{C}}$. Let $\pi: \mathcal{C} \backslash \operatorname{Int}\left(\alpha \cup \alpha^{\prime} \cup \beta \cup \beta^{\prime}\right) \rightarrow \widehat{\mathcal{C}}$ be the natural projection: notice that $\pi$ does not respect the cyclic orders around $\mathcal{C}$ and $\hat{\mathcal{C}}$. The image under $\pi$ of the endpoints of the identified arcs consists of four points $p_{1}, p_{2}, p_{3}, p_{4} \in \hat{\mathcal{C}}$. Denote by $\widehat{\mathcal{C}}_{1}, \widehat{\mathcal{C}}_{2}, \widehat{\mathcal{C}}_{3}$, and $\hat{\mathcal{C}}_{4}$ the four components of $\widehat{\mathcal{C}} \backslash\left\{p_{1}, p_{2}, p_{3}, p_{4}\right\}$; and let $\hat{\mathcal{A}}=\mathcal{A} \backslash\left\{\alpha, \beta, \alpha^{\prime}, \beta^{\prime}\right\}$. Notice that the $\hat{\mathcal{C}}_{i}$ can be regarded as segments in both $\mathcal{C}$ and $\hat{\mathcal{C}}$, and that each is totally ordered by the orientation of $\mathcal{C}$ (and consistently by the induced orientation of $\widehat{\mathcal{C}}$ ). If the $\hat{\mathcal{C}}_{i}$ are labelled so that they appear in the order $\widehat{\mathcal{C}}_{1}, \hat{\mathcal{C}}_{2}, \widehat{\mathcal{C}}_{3}, \hat{\mathcal{C}}_{4}$ around $\mathcal{C}$, then they appear in the reverse order $\hat{\mathcal{C}}_{4}, \widehat{\mathcal{C}}_{3}, \widehat{\mathcal{C}}_{2}, \widehat{\mathcal{C}}_{1}$ around $\hat{\mathcal{C}}$.

If $\gamma \in \hat{\mathcal{A}}$ then $\pi(\gamma)$ is an unlinked arc, with pair $\pi(\phi(\gamma))$, but it need not be maximal. However, it will be shown that there are only finitely many $\gamma \in \hat{\mathcal{A}}$ for which $\pi(\gamma)$ is not maximal, which will establish that $\hat{\mathcal{P}}$ is not finitely linked, as required.

To prove this, suppose that $\Gamma$ is a subset of $\hat{\mathcal{A}}$ such that $\overline{\bigcup_{\gamma \in \Gamma} \pi(\gamma)}$ is an unlinked arc. Suppose that $\Gamma$ contains two arcs $\gamma$ and $\delta$ such that $\pi(\gamma)<\pi(\delta)$ lie in a single $\hat{\mathcal{C}}_{i}$, and $\pi(\phi(\gamma))$ and $\pi(\phi(\delta))$ also lie in a single $\widehat{\mathcal{C}}_{j}$. Then the segment $\llbracket \gamma, \delta \rrbracket$ would be unlinked, contradicting the assumption that the elements of $\mathcal{A}$ are maximal unlinked arcs. Hence $\Gamma$ contains at most three arcs, which are adjacent, and there is some $\gamma \in \Gamma$ such that either $\pi(\gamma)$ or $\pi(\phi(\gamma))$ contains one of the points $p_{i}$. There are therefore at most 24 elements $\gamma$ of $\hat{\mathcal{A}}$ for which $\pi(\gamma)$ is not maximal, as required. 
It remains to show that $\widehat{\mathcal{C}}$ contains no plain arcs. Suppose for a contradiction that it does contain some plain arc $\delta$. Let

$$
\Delta=\{\gamma \in \hat{\mathcal{A}}: \pi(\gamma) \subset \delta\},
$$

and observe that $\phi(\Delta)=\Delta$ since $\delta$ is saturated. Moreover, if $\gamma \in \Delta$ then $\pi(\gamma)$ and $\pi(\phi(\gamma))$ lie in different components $\hat{\mathcal{C}}_{i}$, as otherwise either $[\gamma, \phi(\gamma)]$ or $[\phi(\gamma), \gamma]$ would be a plain arc in $\mathcal{C}$. In particular, $\delta$ contains at least one of the points $p_{i}$ in its interior.

If $\Delta$ is infinite, then pick an infinite segment $\llbracket x, y \rrbracket \subset \Delta$ contained in a single $\widehat{\mathcal{C}}_{i}$. Since $\delta$ is plain, the $\operatorname{arcs}\{\phi(\gamma): \gamma \in \llbracket x, y \rrbracket\}$ lie in the union of the other three components in exactly the opposite order to their pairs in $\llbracket x, y \rrbracket$. Picking a subsegment so that the $\phi(\gamma)$ lie in a single component gives a contradiction to property (i) above.

On the other hand, if $\Delta$ is finite then it contains an arc $\gamma$ such that $\pi(\gamma)$ and $\pi(\phi(\gamma))$ share a common endpoint, which must be one of the points $p_{i}$. Suppose, without loss of generality, that the common endpoint is $p_{1}$, which is the image under $\pi$ of the initial point of $\alpha$ and the final point of $\phi(\alpha)$. If the endpoint of $\gamma$ (respectively $\phi(\gamma)$ ) coincides with the initial point of $\alpha$ then the segment $\llbracket \gamma, \alpha \rrbracket$ (respectively $\llbracket \phi(\gamma), \alpha \rrbracket$ ) gives a contradiction to property (i) above.

The following theorem is the main result of this section. Observe that, in the case where $(P, \mathcal{P})$ is a plain paper-folding scheme, Theorem 50 follows immediately from Theorem 42: $(P, \mathcal{P})$ is finitely linked and is a surface paper folding scheme since the paper space is a sphere; and the scar $G$ is a dendrite, so that the decomposition $G=C \cup \Gamma$ can be realised by taking $C$ to be a point of $G$ and $\Gamma=G$.

Theorem 50 A connected paper-folding scheme $(P, \mathcal{P})$ is a surface paper folding scheme if and only if it is finitely linked. In this case, the scar $G$ is a local dendrite, which can be written as $G=C \cup \Gamma$, where

- $C$ is a finite connected graph in $S$ with the property that any simple closed curve in $C$ is homotopically non-trivial; and

- $\quad \Gamma$ is a union of finitely or countably many disjoint dendrites, with diameters decreasing to 0 , each of which intersects $C$ exactly once.

Proof Suppose first that $(P, \mathcal{P})$ is finitely linked. The identifications $\sim_{\mathcal{P}}$ on $\partial P$ will be done in two steps. First those arising from pairings contained in plain arcs are carried out: applying Moore's theorem as in the proof of Theorem 42, these yield a topological sphere on which the simple closed curve $\mathcal{C}$ bounds a disk whose interior 
contains only points of $\widehat{\mathbb{C}} \backslash P$. Removing this disk and carrying out the remaining (finitely many) identifications along $\mathcal{C}$ yields a topological surface. The details are similar to those of the proof of Theorem 42, and will only be sketched.

As in that proof, regard $\partial P$ as the unit circle in $\widehat{\mathbb{C}}$, so that $P$ is the exterior of the unit disk $\overline{\mathbb{D}}$, which is regarded as the hyperbolic plane. Connect the points of each interior pair in $\partial P$ which is contained in a plain arc with the geodesic joining them, and include also the geodesic $g_{\gamma}$ joining the endpoints of each maximal plain arc $\gamma$. Let $\mathcal{L}$ be the geodesic lamination obtained by taking the closure of the union of these geodesics. For each maximal plain arc $\gamma$, let $D_{\gamma}$ denote the disk bounded by $\gamma \cup g_{\gamma}$. The musc decomposition $\mathcal{G}$ of $\widehat{\mathbb{C}}$ with the following elements then realises the equivalence relation $\sim$ Plain on $\partial P$ corresponding to the set of pairings contained in plain arcs:

(I) Closures of components of $D_{\gamma} \backslash \mathcal{L}$, for each maximal plain arc $\gamma$;

(II) Geodesics in $\mathcal{L}$ (together with endpoints) which are not contained in elements of type (I); and

(III) Points which are not contained in elements of types (I) and (II).

The quotient of $\widehat{\mathbb{C}}$ by $\sim_{\text {Plain }}$ is therefore a topological sphere containing the simple closed curve $\mathcal{C}$ obtained from $\partial P$ by collapsing each maximal plain arc to a point. One complementary component of $\mathcal{C}$ is (the projection of) $\widehat{\mathbb{C}} \backslash\left(P \cup \cup_{\gamma}\right.$ plain $\left.D_{\gamma}\right)$, while the other contains (the projection of) the interior of $P$, together with a dendrite corresponding to each maximal plain arc $\gamma$, attached to $\mathcal{C}$ at a single point. Removing the former complementary component yields a topological disk bounded by $\mathcal{C}$, and carrying out the (finitely many) identifications on $\mathcal{C}$ gives the compact surface $S$ : the bounding curve $\mathcal{C}$ projects to a finite graph $C$ in $S$ in which every simple closed curve is homotopically non-trivial, and the scar $G$ consists of this graph together with the dendrites arising from maximal plain arcs.

For the converse, suppose that $(P, \mathcal{P})$ is not finitely linked. Carry out the identifications realising $\sim_{\text {Plain }}$ as above, and remove the complementary component of $\mathcal{C}$ corresponding to the exterior of $P$. Using Lemma 49, it is possible to carry out repeated additional pairs of identifications, each of which adds 1 to the genus of the resulting surface. It follows that $S$ contains a surface (with boundary) of genus $n$ for all $n$, so that $S$ cannot be a compact surface as required.

The injectivity radius $\bar{r}$ of $G$, which gives the length of the shortest simple closed curve in the scar, will be important in the constructions of Section 5. In the case of a plain paper-folding scheme, where the scar contains no simple closed curves, $\bar{r}$ can be chosen as an arbitrary positive number for these constructions. 
Definition 51 (Injectivity radius of $G$ ) Let $G$ be the scar of a finitely linked and non-plain paper-folding scheme. The injectivity radius $\bar{r}$ of $G$ is defined by

$$
\bar{r}=\frac{1}{2} \inf \left\{|\gamma|_{G}: \gamma \text { is a simple closed curve contained in } G\right\} .
$$

Lemma 52 Let $G$ be the scar of a finitely linked paper-folding scheme $(P, \mathcal{P})$. If $(P, \mathcal{P})$ is not plain, then

(a) The injectivity radius of $G$ satisfies $\bar{r}>0$.

(b) For every $x \in G$ and every $0 \leq r<\bar{r}$, the closed ball $\bar{B}_{G}(x ; r)$ is a dendrite and is thus contractible.

If $(P, \mathcal{P})$ is plain, then statement (b) holds for any $\bar{r}>0$.

Proof Assume first that $(P, \mathcal{P})$ is not plain. Then $\bar{r}>0$ since the graph $C$ of Theorem 50 is finite, and hence there are only finitely many simple closed curves in $G$.

Let $r<\bar{r}$. Then $\bar{B}_{G}(x ; r)$ is a continuum (it is connected since the metric $d_{G}$ is intrinsic) which contains no simple closed curves. Each dendrite in the decomposition of $G$ intersects $\bar{B}_{G}(x ; r)$ in a subcontinuum, and hence in a dendrite (Theorem 8(e)); and similarly $C$ intersects $\bar{B}_{G}(x ; r)$ in a tree. Hence any two distinct points of $\bar{B}_{G}(x ; r)$ are separated by a third point of $\bar{B}_{G}(x ; r)$, establishing by Theorem 8 (a) that it is a dendrite as required.

If $(P, \mathcal{P})$ is plain and $r>0$, then as above $\bar{B}_{G}(x ; r)$ is a subcontinuum of $G$, and hence a dendrite, for every $x \in G$.

\subsection{The metric structure of paper spaces}

Let $S$ be a paper space. Near planar points, $S$ is Euclidean: that is, such points have an open neighborhood which is isometric to an open ball in $\mathbb{R}^{2}$. Similarly, near a regular vertex $x, S$ is isometric to the apex of a cone, with cone angle equal to the sum of the internal angles of the multipolygon $P$ at the points of $\pi^{-1}(x)$. The following definitions formalise this statement. Throughout this section $(P, \mathcal{P})$ is an arbitrary paper-folding scheme with scar $G$ and associated paper space $S$.

Definition 53 (Cone angle at non-singular points) Let $x \in G$ be non-singular with $\pi^{-1}(x)=\left\{\tilde{x}_{1}, \ldots, \tilde{x}_{k}\right\}$, and let $\tilde{\eta}_{i}$ be the internal angle of $P$ at $\tilde{x}_{i}$. The cone angle at $x$ is the number $\eta(x)=\tilde{\eta}_{1}+\cdots+\tilde{\eta}_{k}$.

In particular, the cone angle at a regular point $x \in G$ is $\eta(x)=2 \pi$. 
Definition 54 (Metric cone, see Burago, Burago and Ivanov [3]) Let $X$ be a topological space. The cone Cone $(X)$ over $X$ is the (topological) quotient of $[0, \infty) \times X$ by the equivalence relation which collapses $\{0\} \times X$ to a point, that is, whose only non-trivial class is $\{0\} \times X$. This point in the quotient is the origin or apex of the cone and is also denoted 0 . If $(X, d)$ is a metric space, then it is possible to make Cone $(X)$ into a metric space by defining a distance as follows: if $p=[t, x], q=[s, y] \in \operatorname{Cone}(X)$, set

$$
d_{c}(p, q)= \begin{cases}\sqrt{t^{2}+s^{2}-2 s t \cos (d(x, y))}, & \text { if } d(x, y) \leq \pi \\ t+s, & \text { if } d(x, y) \geq \pi\end{cases}
$$

Let $S_{r}^{1}$ denote the circle of radius $r>0$ in $\mathbb{R}^{2}$ with the intrinsic metric. Then the cone Cone $\left(S_{r}^{1}\right)$ is locally isometric to the plane itself, except at the apex for $r \neq 1$, and Cone $\left(S_{1}^{1}\right)$ is globally isometric to $\mathbb{R}^{2}$. Moreover, two cones on circles Cone $\left(S_{r_{1}}^{1}\right)$, Cone $\left(S_{r_{2}}^{1}\right)$ are globally isometric only if $r_{1}=r_{2}$.

Definition 55 (Conic-flat surface) A conic-flat surface is a metric space which is locally isometric to cones on circles: for every point $x$, there exist $r, \varepsilon>0$ and $c \in$ Cone $\left(S_{r}^{1}\right)$ such that $B(x ; \varepsilon)$ is isometric to $B_{\mathrm{Cone}\left(S_{r}^{1}\right)}(c ; \varepsilon)$.

Theorem 56 (Metric structure of paper spaces)

(a) Let $x$ be a non-singular point of $G$. Then there exists $r>0$ such that $B_{S}(x ; r)$ is isometric to $B_{\mathrm{Cone}\left(S_{\eta(x) / 2 \pi}^{1}\right)}(0 ; r)$. In particular, $S \backslash \mathcal{V}^{s}$ is a conic-flat surface.

(b) The metric $d_{S}$ on a paper space $S$ is strictly intrinsic.

Proof The proof of Part (a) is straightforward and technical and is omitted: this statement is not used in the remainder of the paper. Part (b) is immediate from Lemma 16 and Theorem 15 since the metric $d_{P}$ on $P$ is intrinsic, and $P$ (and hence $S$ ) is compact.

Notice that even if $S$ is a paper surface, the metric on the conic-flat surface $S \backslash \mathcal{V}^{s}$ need not be strictly intrinsic.

\section{The conformal structure on paper surfaces}

If $(P, \mathcal{P})$ is a surface paper-folding scheme and $S$ is the quotient paper surface, then there is a natural conformal structure on the set $\operatorname{Planar}(S)$ of planar points coming from the local Euclidean structure. The question addressed in this section is whether or not this complex structure extends uniquely across non-planar points of $S$. 
The complex structure extends readily across regular vertices of $G$ using the conic-flat structure on $S \backslash \mathcal{V}^{s}$ described in Section 4.3: at a cone point of angle $\eta$, the map $z \mapsto z^{2 \pi / \eta}$ can be used to introduce conformal coordinates. Thus the case of interest is that of isolated singular points. Theorem 59 below provides a criterion for the complex structure to extend uniquely across such a point. In particular, if all singular points are isolated, and this criterion holds at each of them, then $S$ is a Riemann surface. Similar conditions which guarantee that the complex structure extends uniquely across a more general singular set can also be obtained, and this will be the subject of a forthcoming paper.

The question of whether the complex structure extends uniquely across an isolated singular point is clearly a local one, at least as far as $S$ is concerned. However, both the results and the techniques of this section will be central in Section 6, where a global modulus of continuity for a uniformising map is obtained. A more global approach is therefore taken than is necessary for the results of this section alone.

Again, the local nature of the problem means that there is no essential distinction between plain and non-plain surface paper-foldings. For simplicity of exposition, however, the details of the construction and proof (from Section 5.3 onwards) will be carried out in the plain case: the minor modifications needed for non-plain examples will be described at the end of Section 5.6.

The main theorem is stated in Section 5.1, and examples of its application are given in Section 5.2. The idea of the proof (which was inspired by similar constructions in Earle-Gardiner [8], de Carvalho-Hall [6] and de Carvalho [5]; see also ChamanaraGardiner-Lakic [4]) is to construct a nested sequence of annuli with divergent module sum zooming down to the singular point $q$, and to apply Lemmas 22 and 23. In order to be able to construct these annuli in a way which makes it possible to estimate their modules, a foliated collaring of the polygon $P$ is used: this is described in Section 5.3. The annuli themselves are constructed in Section 5.4, and some technical lemmas needed to estimate their modules are given in Section 5.5, before the proof of Theorem 59 is given in Section 5.6.

\subsection{Statement of results}

Definitions 57 ( $m(q ; r), n(q ; r)$, planar radius) Let $G$ be the scar of a paper-folding scheme $(P, \mathcal{P}), q \in G$, and $r>0$. Recall (Notation 10) that $C_{G}(q ; r)$ denotes the set of points of $G$ at $d_{G}$-distance exactly $r$ from $q$. Define

$$
n(q ; r):=\# C_{G}(q ; r) \in \mathbb{N} \cup\{\infty\} .
$$


A radius $r>0$ is said to be planar for $q$, or $q$-planar, if all points of $C_{G}(q ; r)$ are planar.

Define also

$$
m(q ; r):=\mathrm{m}_{G}\left(B_{G}(q ; r)\right) \in\left(0, \mathrm{~m}_{G}(G)\right] .
$$

In the statement and proof of the following result, recall (Definition 51 and Lemma 52) that $\bar{r}$ denotes the injectivity radius of $G$ (or an arbitrary positive number if $(P, \mathcal{P})$ is plain), and that $\bar{B}_{G}(q ; r)$ is a dendrite for all $q \in G$ and all $r<\bar{r}$.

Lemma 58 If $r \in(0, \bar{r})$ is a planar radius for $q \in G$, then $n(q ; r)$ is finite and is locally constant on both variables $q, r$. Moreover, given $q \in G$, the set of radii which are not planar for $q$ is a closed subset of $(0, \bar{r})$ of measure zero.

Proof Recall (Lemma 33(a)) that the set $\overline{\mathcal{V}}$ of non-planar points is a compact subset of $G$ with zero $\mathrm{m}_{G}$-measure. If $r>0$ is planar for $q$ there is therefore some $\delta>0$ such that $|d(s, q)-r|>\delta$ for all $s \in \overline{\mathcal{V}}$.

It follows that for each $x \in C_{G}(q ; r)$, the ball $I_{x}=B_{G}(x ; \delta)$ is isometric to an interval of length $2 \delta$. Since $\bar{B}_{G}(q ; r)$ is a dendrite and the metric $d_{G}$ is strictly intrinsic, $I_{x}$ cannot contain any other point of $C_{G}(q ; r)$ (otherwise the unique shortest path from $q$ to one of the points would be strictly shorter than that to the other). This establishes that $n(q ; r)$ is finite, since $\mathrm{m}_{G}(G)$ is finite.

For the local constantness of $n(q ; r)$, observe that if $d_{G}\left(q, q^{\prime}\right)<\delta / 4$ and $\left|r-r^{\prime}\right|<\delta / 4$, then each $I_{x}$ contains a point of $C_{G}\left(q^{\prime} ; r^{\prime}\right)$, and hence $n\left(q^{\prime} ; r^{\prime}\right) \geq n(q ; r)$. On the other hand, since $\left|d\left(s, q^{\prime}\right)-r^{\prime}\right|>\delta / 2$ for all $s \in \overline{\mathcal{V}}$, the same argument works the other way round to show that $n(q ; r) \geq n\left(q^{\prime} ; r^{\prime}\right)$ as required.

$\overline{\mathcal{V}}$ has zero $\mathrm{m}_{G}-$ measure, and hence zero $\mu_{G}^{1}-$ measure by Lemma 33(c). Since the function $G \rightarrow \mathbb{R}$ defined by $s \mapsto d_{G}(q, s)$ is distance non-increasing, it follows from Lemma 20 that the set $\left\{d_{G}(q, s): s \in \overline{\mathcal{V}}\right\}$ of non-planar radii for $q$ has zero measure.

Since $\overline{\mathcal{V}}$ is compact and $x \mapsto d_{G}(q, x)$ is a continuous map $G \rightarrow \mathbb{R}$, the set of nonplanar radii together with zero is closed in $\mathbb{R}$, and hence the set of non-planar radii is closed in $(0, \bar{r})$.

It is now possible to state one of the main theorems of this paper:

Theorem 59 Let $(P, \mathcal{P})$ be a surface paper-folding scheme with associated paper surface $S$ and scar $G \subset S$. If $q \in G$ is an isolated singular point, then the complex structure on $S \backslash \mathcal{V}^{s}$ extends uniquely across $q$ provided that

$$
\int_{0} \frac{\mathrm{d} r}{m(q ; r)+r \cdot n(q ; r)}=\infty \text {. }
$$


In particular, if all singular points are isolated and the integral condition above holds for every one of them, then $S$ is a compact Riemann surface.

Remark 60 The integral in the statement diverges at planar points in $G$ and at regular vertices. At a regular $k$-vertex with $k<\infty$, or at a planar point $(k=2), n(q ; r)=k$ and $m(q ; r)=2 k r$ for all sufficiently small $r$. Therefore

$$
\int_{\varepsilon} \frac{\mathrm{d} r}{m(q ; r)+r \cdot n(q ; r)}
$$

goes like $-\ln \varepsilon$. The proof of Theorem 59 presented in this section also shows that if $q \in G$ is a non-singular point, then the complex structure on $S \backslash\left(\mathcal{V}^{s} \cup\{q\}\right)$ extends uniquely across $q$.

\subsection{Example: $\infty$-od singularities}

A case of special interest is that of $\infty$-od singularities: they have already appeared in Example 35 (see also Example 43), and are common in dynamical applications.

Definition 61 ( $\infty$-od singularity) An isolated singularity $q \in G$ is an $\infty$-od singularity if there is some $r_{0}>0$ such that $\bar{B}_{G}\left(q ; r_{0}\right)$ is an $\infty$-od (Definition 12).

Suppose that $q \in G$ is an $\infty$-od singularity. Then if $0<r \leq r_{0}$, every point $x \in C_{G}(q ; r)$ is joined to $q$ by a unique arc $\overline{x q}$ all of whose interior points are planar and whose length is $r$, and these arcs intersect only at $q$. By Lemma 33(d), $\mathrm{m}_{G}(\overline{x q})=2 r$. There are as many such arcs in $\bar{B}_{G}(q ; r)$ as there are points in $C_{G}(q ; r)$, from which it follows that $m(q ; r) \geq 2 r \cdot n(q ; r)$. This proves the following

Corollary 62 For the complex structure on $\mathcal{R}$ to extend uniquely across an $\infty$-od singularity $q \in G$ it is sufficient that

$$
\int_{0} \frac{\mathrm{d} r}{m(q ; r)}=\infty
$$

Example 63 Consider Example 35. Notice that in the criterion of Corollary 62 only the measure $m(q ; r)=\mathrm{m}_{G}\left(B_{G}(q ; r)\right)$ is taken into account and the way in which the identifications about the $\infty$-od singularity $q$ are done is not relevant. Thus there is no distinction between the two ways of arranging the folds in the example, since the resulting scars are isometric. What is important is the asymptotics of the decreasing sequence $\left(a_{n}\right)$ of fold lengths. 
Write $N(r):=n(q ; r)=\max \left\{n: a_{n} \geq r\right\}$ for $r>0$. Then

$$
m(q ; r)=2 r \cdot N(r)+2 \sum_{n>N(r)} a_{n} .
$$

Suppose first that $a_{n} \asymp 1 / n^{k}$, for some $k>1$ : that is, $C_{1} / n^{k} \leq a_{n} \leq C_{2} / n^{k}$ for some positive constants $C_{1}$ and $C_{2}$. In this case, $n \leq\left(C_{1} / r\right)^{1 / k}$ implies $a_{n} \geq r$, and $n>\left(C_{2} / r\right)^{1 / k}$ implies $a_{n}<r$, so that $\left(C_{1} / r\right)^{1 / k} \leq N(r) \leq\left(C_{2} / r\right)^{1 / k}$. Using (10) with the lower bound for $N(r)$ in the first term, the upper bound for $N(r)$ in the second, and lower bounds for $a_{n}$ gives $m(q ; r) \geq C_{3} r^{1-1 / k}$ for sufficiently small $r$, so that

$$
\int_{0} \frac{\mathrm{d} r}{m(q ; r)} \leq \int_{0} \frac{\mathrm{d} r}{C_{3} r^{1-1 / k}}<\infty .
$$

This means the criterion cannot be used to guarantee that the complex structure extends uniquely across the point $q$, and the authors know no way of determining whether or not it does so extend.

If, on the other hand, $a_{n} \asymp 1 / \lambda^{n}$ for some $\lambda>1$, then

$$
\frac{\ln \left(C_{1} / r\right)}{\ln \lambda} \leq N(r) \leq \frac{\ln \left(C_{2} / r\right)}{\ln \lambda},
$$

and it follows from (10) that

$$
\begin{aligned}
\frac{1}{2} m(q ; r) & \leq \frac{r}{\ln \lambda} \ln \frac{C_{2}}{r}+\frac{C_{2}}{\lambda N(r)(\lambda-1)} \\
& \leq \frac{r}{\ln \lambda}\left(\ln C_{2}+\ln \frac{1}{r}\right)+\frac{C_{2} r}{C_{1}(\lambda-1)} \\
& \leq C_{3} r \ln \frac{1}{r} \quad \text { for } r \text { sufficiently small, }
\end{aligned}
$$

so that

$$
2 \int_{0} \frac{\mathrm{d} r}{m(q ; r)} \geq \int_{0} \frac{\mathrm{d} r}{C_{3} r \ln (1 / r)}=\infty .
$$

This time Corollary 62 applies and thus the quotient space is a complex sphere.

Remark 64 If the Cantor construction of Example 35 is that of the standard middle thirds Cantor set, then listing the edges of the $\infty$-od in decreasing order gives

$$
a_{n}=\frac{1}{6 \cdot 3^{k}} \quad \text { for } \quad 2^{k} \leq n<2^{k+1},
$$

which implies that

$$
\frac{1}{6}\left(\frac{1}{n}\right)^{\frac{\ln 3}{\ln 2}} \leq a_{n} \leq \frac{1}{2}\left(\frac{1}{n}\right)^{\frac{\ln 3}{\ln 2}}
$$


Thus this is an example in which the hypothesis of Corollary 62 is not satisfied.

\subsection{Foliated collaring of $P$}

Let $(P, \mathcal{P})$ be a surface paper-folding scheme with associated paper surface $S$ and scar $G$, and let $q$ be an isolated singular point of $G$. In order to show that the complex structure on $S \backslash \mathcal{V}^{s}$ extends uniquely across $q$ under appropriate conditions, a nested sequence of annuli zooming down to $q$ will be defined. These annuli will be constructed in a foliated neighborhood of $q$ arising from a foliated collar of $P$, which is described in this section.

For simplicity of exposition, the case in which $(P, \mathcal{P})$ is plain will be considered first. Thus $P$ is a single polygon, $G$ is a dendrite, and $S$ is a topological sphere (Definitions 34 and Theorem 42).

The collar $\tilde{Q}$ of $P$ is constructed as a union of trapezoids whose bases are the sides of $P$; whose vertical sides bisect the angles at the vertices of $P$; and which have fixed height $\bar{h}$, chosen small enough that the trapezoids are far from degenerate and intersect only along their vertical sides. It has a horizontal foliation by leaves parallel to $\partial P$, and a vertical foliation by leaves joining the base and the top of each trapezoid: see Figure 4. The following paragraphs define the collar and foliations more carefully, and set up the notation which will be used.
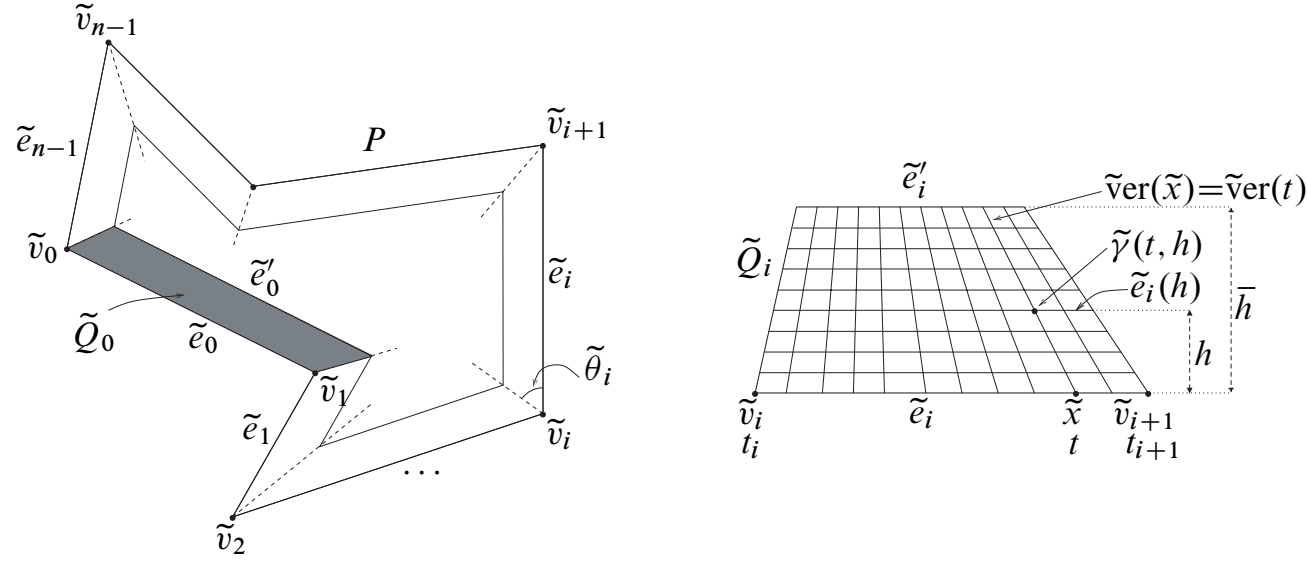

Figure 4: The collar $\widetilde{Q}$ and its foliations

5.3.1 The collar $\widetilde{Q}$ Choose a labeling $\widetilde{v}_{i}(i=0, \ldots, n-1)$ of the vertices of $P$ listed counterclockwise around $\partial P$, and let $\widetilde{e}_{i}$ be the side of $P$ with endpoints $\widetilde{v}_{i}$ and $\widetilde{v}_{i+1}$ (here and throughout, subscripts on cyclically ordered objects are taken $\bmod n$ ). Write $L=|\partial P|$, and let $\tilde{\gamma}_{0}:[0, L) \rightarrow \partial P$ be the order-preserving parametrisation 
of $\partial P$ by arc-length with $\tilde{\gamma}_{0}(0)=\widetilde{v}_{0}$. Denote by $t_{i} \in[0, L)$ the parameter with $\tilde{\gamma}_{0}\left(t_{i}\right)=\tilde{v}_{i}$.

A trapezoid is a quadrilateral in $\mathbb{R}^{2}$ with two parallel sides, which are called its base and its top: the other sides are called vertical sides. The height of the trapezoid is the distance between the parallel lines containing its base and its top.

Pick a height $\bar{h}$ small enough that the trapezoids $\widetilde{Q}_{i}$ which have bases $\widetilde{e}_{i}$, heights $\bar{h}$, and vertical sides along the rays bisecting the internal angles of $P$ satisfy:

(a) The lengths of the tops of the trapezoids are between half and twice the lengths of their bases; and

(b) The trapezoids intersect only along their vertical sides.

This height $\bar{h}$ is an important quantity in the construction, and will remain fixed throughout the remainder of the section. Denote the top of $\widetilde{Q}_{i}$ by $\widetilde{e}_{i}^{\prime}$, and let $\tilde{\theta}_{i}$ be half of the internal angle of $\partial P$ at $\widetilde{v}_{i}$ : thus the internal angles of $\widetilde{Q}_{i}$ at the endpoints of its base are $\tilde{\theta}_{i}$ and $\tilde{\theta}_{i+1}$. Condition (a) above is that

$$
\frac{1}{2} \leq \frac{\left|\widetilde{e}_{i}^{\prime}\right|}{\left|\widetilde{e}_{i}\right|} \leq 2 \quad \text { for } i=0, \ldots, n-1 .
$$

Let

$$
\widetilde{Q}=\bigcup_{i=0}^{n-1} \widetilde{Q}_{i},
$$

a closed collar neighborhood of $\partial P$ in $P$.

5.3.2 The foliations Hor and $\tilde{V}$ er For each $h \in[0, \bar{h}]$, let $\widetilde{e}_{i}(h) \subset \widetilde{Q}_{i}$ be the segment parallel to the base of $\widetilde{Q}_{i}$ at height $h$, so that $\tilde{e}_{i}(0)=\widetilde{e}_{i}$ and $\widetilde{e}_{i}(\bar{h})=\widetilde{e}_{i}^{\prime}$. Then the union $\tilde{h}$ or $(h)$ of these segments is a polygonal simple closed curve: these simple closed curves are the leaves of the horizontal foliation

$$
\text { Hor }=\{\tilde{\operatorname{hor}}(h): h \in[0, \bar{h}]\}
$$

of $\widetilde{Q}$. The parameter $h$ is called the height of the leaf $\tilde{h}$ or $(h)$.

Write

$$
\widetilde{Q}(h)=\bigcup_{h^{\prime} \in[0, h]} \tilde{\text { hor }}\left(h^{\prime}\right),
$$

the subset of $\widetilde{Q}$ consisting of leaves with heights not exceeding $h: \widetilde{Q}(h) \subset \widetilde{Q}=\widetilde{Q}(\bar{h})$ is therefore also a closed collar neighborhood of $\partial P$ for each $h \in(0, \bar{h}]$. 
To construct the vertical foliation, let $\varphi_{i}: \widetilde{e}_{i} \rightarrow \widetilde{e}_{i}^{\prime}$ be the orientation-preserving scaling from $\tilde{e}_{i}$ to $\tilde{e}_{i}^{\prime}$. For each $\tilde{x}=\tilde{\gamma}_{0}(t) \in \widetilde{e}_{i}$, denote by $\tilde{v} \operatorname{er}(\tilde{x})$ or $\operatorname{ver}(t)$ the straight line segment which joins $\tilde{x}$ to $\varphi_{i}(\tilde{x})$. These segments are the leaves of the vertical foliation

$$
\tilde{\operatorname{Ver}}=\{\tilde{\operatorname{ver}}(t): t \in[0, L)\}
$$

of $\widetilde{Q}$.

Define $\tilde{\theta}:[0, L) \backslash\left\{t_{0}, \ldots, t_{n-1}\right\} \rightarrow(0, \pi)$ by setting $\tilde{\theta}(t)$ to be the angle between $\partial P$ and $\tilde{v} \operatorname{ver}(t)$ at $\tilde{\gamma}_{0}(t)$ : that is, the angle between the oriented side of $\partial P$ containing $\tilde{\gamma}_{0}(t)$ and the leaf $\operatorname{ver}(t)$ pointing into $P$. This function has well-defined limits as $t$ approaches each $t_{i}$ from the left or the right: $\tilde{\theta}\left(t_{i}^{-}\right)=\pi-\tilde{\theta}_{i}$, and $\tilde{\theta}\left(t_{i}^{+}\right)=\tilde{\theta}_{i}$. The notation $\tilde{\theta}(\tilde{x})=\tilde{\theta}(t)$ will also be used when $\tilde{x}=\tilde{\gamma}_{0}(t)$.

The foliations $\tilde{H}$ or and $\tilde{V}$ er yield a parametrisation

$$
\tilde{\gamma}:[0, L) \times[0, \bar{h}] \rightarrow \widetilde{Q}
$$

of $\widetilde{Q}$, where $\tilde{\gamma}(t, h)$ is the unique point of $\tilde{\operatorname{ver}}(t) \cap \tilde{\operatorname{hor}}(h)$.

For each $h \in(0, \bar{h}]$, denote by $\tilde{\psi}_{h}: \widetilde{Q}(h) \rightarrow \partial P$ the retraction of $\widetilde{Q}(h)$ onto $\partial P$ which slides each point along its vertical leaf:

$$
\tilde{\psi}_{h}\left(\gamma\left(t, h^{\prime}\right)\right)=\gamma(t, 0) \quad\left(\text { all } t \in[0, L) \text { and } h^{\prime} \in[0, h]\right) .
$$

In particular, $\tilde{\psi}_{\bar{h}}$ is a retraction which squashes all of $\widetilde{Q}$ onto $\partial P$.

5.3.3 The foliations on $S$ The projections to the paper surface $S$ of the structures defined above are denoted by removing tildes (see Figure 5). Thus $Q=\pi(\widetilde{Q})$ is a closed disk neighborhood of the scar $G$, and similarly $Q(h)=\pi(\widetilde{Q}(h))$ is a closed subdisk neighborhood for each $h \in(0, \bar{h}]$. $Q$ has horizontal and vertical foliations Hor $=\pi(\tilde{H}$ or $)$ and Ver $=\pi(\tilde{V} e r)$. The leaves of Hor are projections of leaves of Hor: $\operatorname{hor}(h)=\pi(\tilde{\operatorname{hor}}(h))$. The leaves of Ver, however, are unions of projections of leaves of Ver: for each $x \in G$, the leaf of Ver containing $x$ is defined to be

$$
\operatorname{ver}(x):=\bigcup\left\{\pi(\tilde{\operatorname{ver}}(\tilde{x})): \tilde{x} \in \pi^{-1}(x)\right\} .
$$

Thus $\operatorname{ver}(x)$ is an arc if and only if $\# \pi^{-1}(x) \leq 2$. If $x$ is a $k$-vertex for $k>2$ then $\operatorname{ver}(x)$ is a star with $k$ branches. Note, however, that if $x$ is an $\infty$-vertex then $\operatorname{ver}(x)$ is not an $\infty$-od in the sense of Definition 12, since the lengths of its branches do not converge to zero.

The disks $Q(h)$ for $0<h \leq \bar{h}$ are similarly foliated by horizontal leaves hor $\left(h^{\prime}\right)$ with $0<h^{\prime} \leq h$, and vertical leaves $\operatorname{ver}_{h}(x)$, which are the leaves $\operatorname{ver}(x)$ trimmed at their intersection with hor $(h)$. 


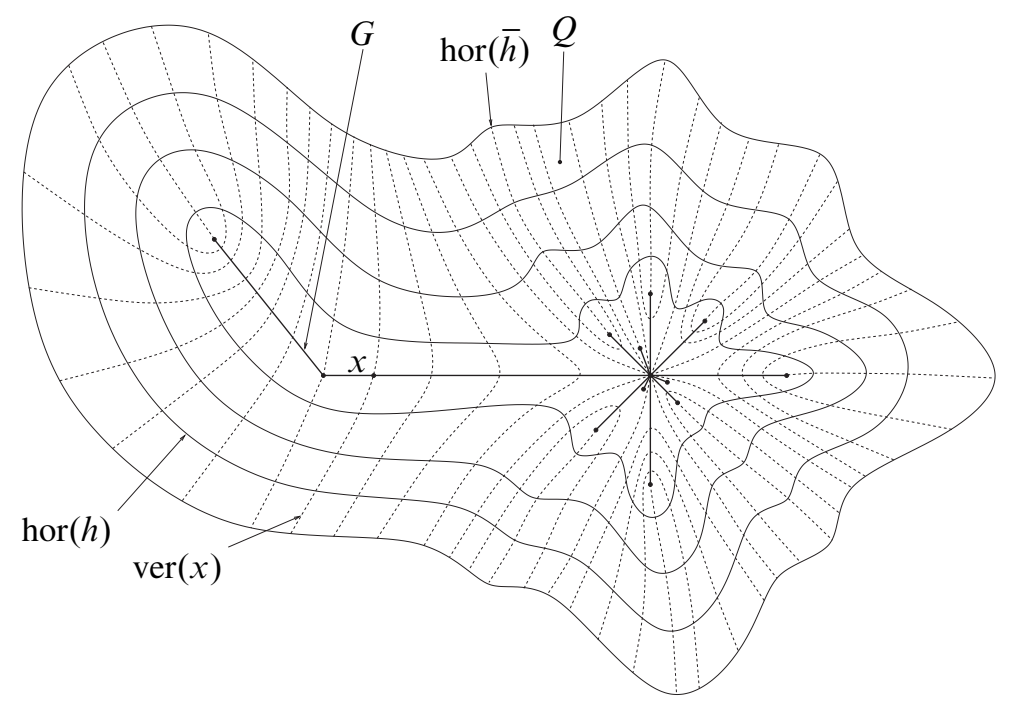

Figure 5: The foliations projected to $S$

The composition $\gamma:=\pi \circ \tilde{\gamma}:[0, L) \times[0, \bar{h}] \rightarrow Q$ parametrises $Q$, although it is not injective on $G$. Notice that because the retractions $\widetilde{\psi}_{h}: \widetilde{Q}(h) \rightarrow \partial P$ fix $\partial P$ pointwise, the compositions

$$
\psi_{h}:=\pi \circ \tilde{\psi}_{h} \circ \pi^{-1}: Q(h) \rightarrow G
$$

are well-defined retractions of $Q(h)$ onto $G$.

\subsection{The system of annuli $\operatorname{Ann}(q ; r, s)$}

Let $q$ be a point of the scar $G$. In this section annuli $\operatorname{Ann}(q ; r, s)$ about $q$ will be constructed for each pair of $q$-planar radii $r<s$. The annuli will be defined as differences of two topological closed disks: $\operatorname{Ann}(q ; r, s)=\operatorname{Int}(D(q ; s)) \backslash D(q ; r)$.

There are naturally two parameters $r$ and $h$ involved in constructing such disks about $q$ using the foliations of $Q$, which describe respectively the vertical and the horizontal leaves which will form its boundary. Here, though, the ratio between these parameters will be fixed. The ratio is determined by a number $\bar{r}>0$, which in this section can be chosen arbitrarily: in the non-plain case, however, it will become the injectivity radius of $G$ (Definition 51).

Choose $\bar{r}>0$, then, and define a function $h:[0, \bar{r}] \rightarrow[0, \bar{h} / 2]$ by

$$
h(r):=\left(\frac{\bar{h}}{2 \bar{r}}\right) r,
$$

which will fix the parameter $h$ given the radius $r$. 
Definition $65(D(q ; r))$ Let $q \in G$ and let $r \in(0, \bar{r}]$. The subset $D(q ; r)$ of $Q$ is defined by

$$
D(q ; r):=\psi_{h(r)}^{-1}\left(\bar{B}_{G}(q ; r)\right) .
$$

Alternatively, $D(q ; r)$ is the intersection of $Q(h(r))$ with the union of the vertical leaves $\operatorname{ver}(x)$ with $d_{G}(q, x) \leq r$.

Lemma 66 Let $q \in G$ and $r \in(0, \bar{r}]$ be a $q$-planar radius. Write $n=n(q ; r)$ and $h=h(r)$. Then $D(q ; r)$ is a topological closed disk, whose boundary is composed of $n$ disjoint subarcs of the horizontal leaf hor $(h)$, and the $n$ trimmed vertical leaves $\operatorname{ver}_{h}(x)$ with $x \in C_{G}(q ; r)$.

Proof (See Figure 6.) Write $C_{G}(q ; r)=\left\{x_{0}, \ldots, x_{n-1}\right\}$. By definition, the boundary $\partial_{Q(h)} D(q ; r)$ of $D(q ; r)=\psi_{h}^{-1}\left(\bar{B}_{G}(q ; r)\right) \subset Q(h)$ in $Q(h)$ is contained in $\psi_{h}^{-1}\left(C_{G}(q ; r)\right)$. Moreover, since $r$ is $q$-planar, every neighborhood of each point of $C_{G}(q ; r)$ contains both points which are closer to $q$ and points which are further away (see the proof of Lemma 58), and hence

$$
\partial_{Q(h)} D(q ; r)=\psi_{h}^{-1}\left(C_{G}(q ; r)\right)=\bigcup_{i=0}^{n-1} \psi_{h}^{-1}\left(x_{i}\right) .
$$

Since the points $x_{i}$ are planar, each $\psi_{h}^{-1}\left(x_{i}\right)=\operatorname{ver}_{h}\left(x_{i}\right)$ is an arc which intersects hor $(h)=\partial_{S} Q(h)$ exactly at its endpoints: that is, a cross cut in $Q(h)$. For each $i, Q(h) \backslash \operatorname{ver}_{h}\left(x_{i}\right)$ has exactly two components, one of which intersects $G$ in the complement of $\bar{B}_{G}(q ; r)$. Therefore every $\operatorname{ver}_{h}\left(x_{j}\right)$ with $j \neq i$ is contained in the same component as $q$. It follows that $\partial_{S} D(q ; r)$ is the simple closed curve composed of the $\operatorname{arcs}_{\operatorname{ver}_{h}}\left(x_{i}\right)$ and the $n$ subarcs of hor $(h)$ joining the endpoints of consecutive cross cuts in the cyclic order around hor $(h)$.

The annuli which will be used in the proof of Theorem 59 can now be defined.

Definition $67(\operatorname{Ann}(q ; r, s))$ Let $q \in G$, and $r, s \in(0, \bar{r}]$ be $q$-planar radii with $r<s$. The subset $\operatorname{Ann}(q ; r, s)$ of $Q$ is defined by

$$
\operatorname{Ann}(q ; r, s)=\operatorname{Int}(D(q ; s)) \backslash D(q ; r) .
$$

By Lemma 66, and since $D(q ; r) \subset \operatorname{Int}(D(q ; s)), \operatorname{Ann}(q ; r, s)$ is an open annular region with $q$ in its bounded complementary component (the complementary component not containing $\partial Q$ ). 


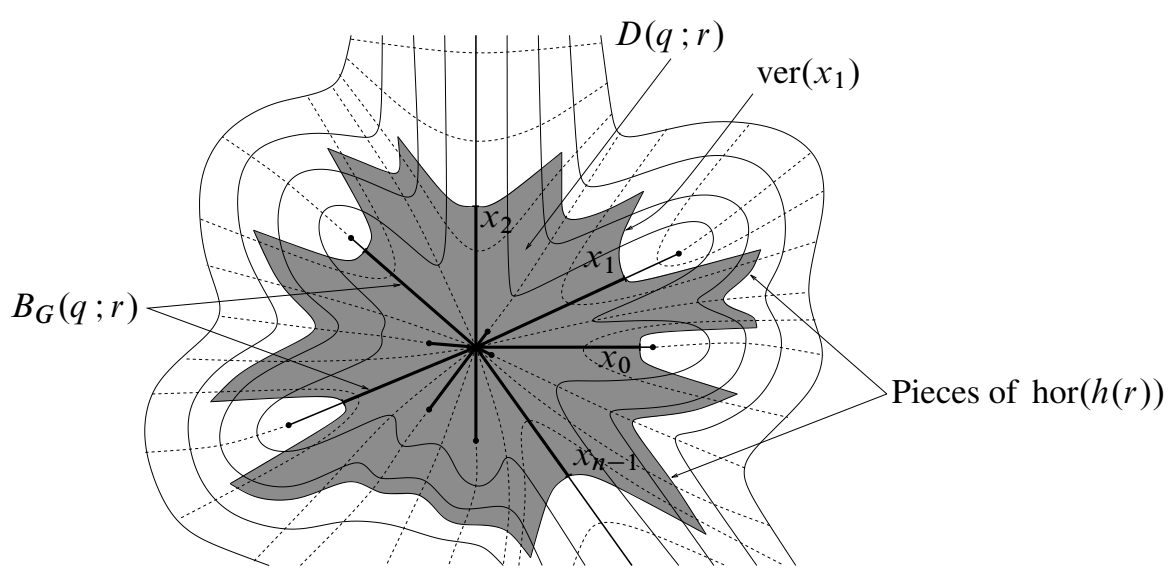

Figure 6: The disk $D(q ; r)$

\subsection{Polygon constants and geometry of the trapezoid construction}

The goal now is to find lower bounds on the conformal modules of the annuli $\operatorname{Ann}(q ; r, s)$. In order to use (3), it is necessary to estimate the distance in $S$ between the two boundary components of the annulus, and the area of the annulus. This will be done by lifting to the polygon $P$, where the preimage of the disk $D(q ; r)$ is a union of polytrapezoids.

The bounds will be expressed in terms of the polygon constants of $P$ : this will make it possible, in Section 6, to obtain uniform moduli of continuity for uniformising maps in families of polygons with bounded constants.

Definition 68 (Polygon constants) The polygon constants of $P$ are the numbers $\bar{h}$, $\bar{r}$, and $|\partial P|$.

\section{Remarks 69}

(a) Of course there is some freedom in the choice of $\bar{h}$, and total freedom in the choice of $\bar{r}$ in the plain case. The important point is that, in order to prevent the constructions from becoming degenerate in families of examples, $\bar{r}$ and $\bar{h}$ should be bounded away from zero, and $|\partial P|$ should be bounded above.

(b) The internal semi-angles $\tilde{\theta}_{i}$ of $P$ are bounded away from 0 and $\pi$ in terms of the polygon constants, since the vertical edge of $\widetilde{Q}_{i-1}$ and $\widetilde{Q}_{i}$ emanating from $\widetilde{v}_{i}$ has length $\bar{h} / \sin \tilde{\theta}_{i}$ and is contained in $P$ : hence

$$
\sin \tilde{\theta}_{i} \geq \frac{2 \bar{h}}{|\partial P|} \quad \text { for } i=0, \ldots n-1 .
$$


The following elementary plane geometry lemma bounds the derivative of the cotangent of the function $\tilde{\theta}(\tilde{x})$ of Section 5.3.2.

Lemma 70 Let $\tilde{x}$ lie in the interior of one of the sides $\tilde{e}_{i}$ of $P$. Then

$$
\left|\frac{\mathrm{d}}{\mathrm{d} \tilde{x}} \cot \tilde{\theta}(\tilde{x})\right| \leq \frac{1}{\bar{h}}
$$

Proof Suppose first that $\left|\widetilde{e}_{i}\right|>\left|\widetilde{e}_{i}^{\prime}\right|$. Extend the vertical sides of the trapezoid $\widetilde{Q}_{i}$ to form a triangle of height $H$ : by (11), $H \geq 2 \bar{h}$. The vertical leaf through $\tilde{x} \in \widetilde{e}_{i}$ is a segment of the straight line joining $\tilde{x}$ to the apex of this triangle.

Now if $\tilde{x}_{1}$ and $\tilde{x}_{2}$ lie in $\tilde{e}_{i}$, then the distance between $\tilde{x}_{1}$ and $\tilde{x}_{2}$ is given by $H\left|\cot \left(\tilde{\theta}\left(\tilde{x}_{1}\right)\right)-\cot \left(\tilde{\theta}\left(\tilde{x}_{2}\right)\right)\right|$. Hence

$$
\left|\frac{\mathrm{d}}{\mathrm{d} \tilde{x}} \cot \tilde{\theta}(\tilde{x})\right|=\frac{1}{H} \leq \frac{1}{2 \bar{h}} .
$$

If $\left|\widetilde{e}_{i}\right|<\left|\widetilde{e}_{i}^{\prime}\right|$, then again extend the vertical sides of $\widetilde{Q}_{i}$ to form a triangle of height $H \geq$ $2 \bar{h}$. In this case the distance between $\tilde{x}_{1}$ and $\tilde{x}_{2}$ is given by $(H-\bar{h}) \mid \cot \left(\tilde{\theta}\left(\tilde{x}_{1}\right)\right)-$ $\cot \left(\tilde{\theta}\left(\tilde{x}_{2}\right)\right) \mid$, so that

If $\left|\widetilde{e}_{i}\right|=\left|\widetilde{e}_{i}^{\prime}\right|$, then $\tilde{\theta}(\tilde{x})$ is constant on $\widetilde{e}_{i}$.

$$
\left|\frac{\mathrm{d}}{\mathrm{d} \tilde{x}} \cot \tilde{\theta}(\tilde{x})\right|=\frac{1}{H-\bar{h}} \leq \frac{1}{\bar{h}} .
$$

Now let $q$ be a point of $G$, which will remain fixed throughout the remainder of this section (so the dependence of many objects on $q$ will not be explicitly noted). Let $\left(r_{1}, r_{2}\right) \subset$ $(0, \bar{r}]$ be an interval of $q$-planar radii, and choose $r \in\left(r_{1}, r_{2}\right)$. As before (see Lemma 66 and its proof), write $n=n(q ; r)$, and let $C_{G}(q ; r)=\left\{x_{0}(r), \ldots, x_{n-1}(r)\right\}$, labelling its points in the counterclockwise direction around hor $(h(r))$.

Since $G$ is a dendrite, each of the points $x_{i}(r)$ is connected to $x_{i+1}(r)$ by a unique $\operatorname{arc} \alpha_{i}=\left[x_{i}(r), x_{i+1}(r)\right]_{G}$ in $G$ (Theorem 8(b)). These arcs, which are cross cuts in $D(q ; r)$, form the boundaries in $D(q ; r)$ of $n$ closed subdisks $T_{i}(q ; r)(0 \leq i \leq$ $n-1)$ (see Figure 7). As a subset of $S, T_{i}(q ; r)$ is bounded by:

- The $\operatorname{arc} \alpha_{i}$;

- $\operatorname{ver}_{h(r)}^{+}\left(x_{i}(r)\right)$ and $\operatorname{ver}_{h(r)}^{-}\left(x_{i+1}(r)\right)$, segments of vertical leaf with one endpoint at $x_{i}(r)$ and $x_{i+1}(r)$ respectively - denote their other endpoints $z_{i}^{+}(r)$ and $z_{i+1}^{-}(r) ;$ and

- $\Lambda_{i}(r)$, an arc of $h o r(h(r))$ from $z_{i}^{+}(r)$ to $z_{i+1}^{-}(r)$ in the counterclockwise direction. 
Since the union of the $\operatorname{arcs} \alpha_{i}$ is a tree whose set of endpoints is $C_{G}(q ; r) \subset \partial D(q ; r)$,

$$
D(q ; r)=\bigcup_{i=0}^{n-1} T_{i}(q ; r),
$$

and the subsets $T_{i}(q ; r)$ intersect only along the $\operatorname{arcs} \alpha_{i}$. (If a topological disk $D$ contains a tree $T$ which intersects $\partial D$ precisely at its endpoints, then components of $D \backslash T$ correspond bijectively to pairs of consecutive endpoints on $\partial D$.)

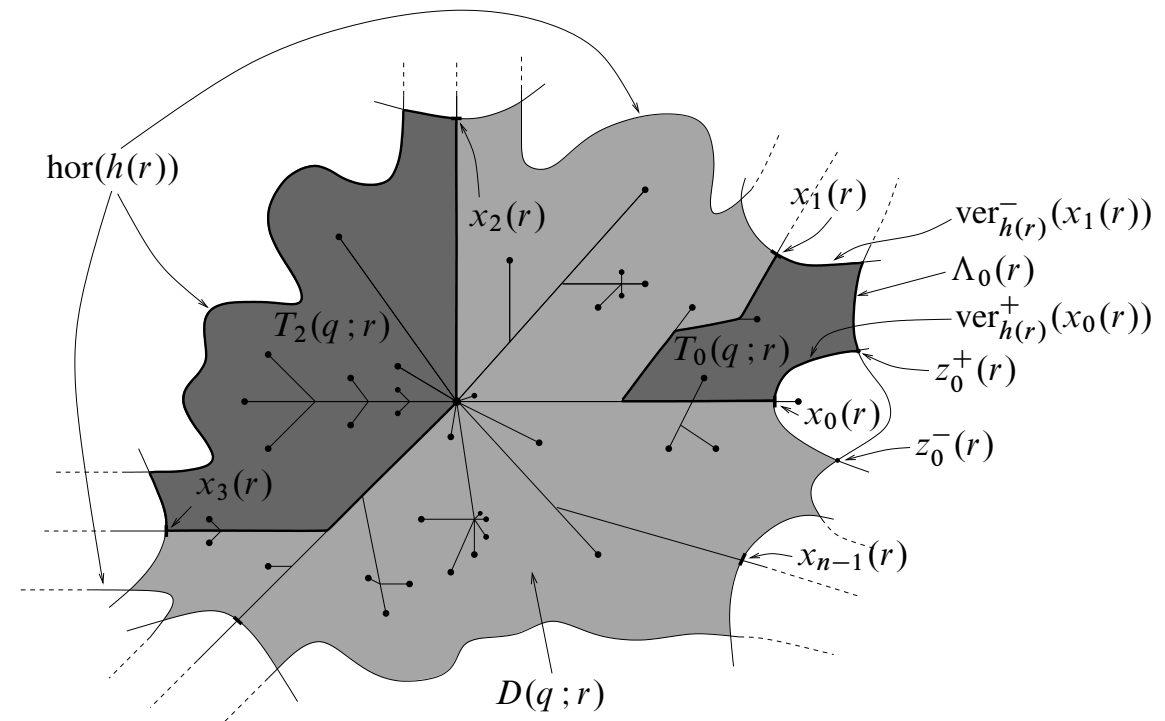

Figure 7: The regions $T_{i}(q ; r)$

Now $\Lambda_{i}(r)$ lies in $Q \backslash G$, and hence has a well-defined preimage $\tilde{\Lambda}_{i}(r)=\pi^{-1}\left(\Lambda_{i}(r)\right)$, which is an arc of $\tilde{h} \operatorname{ror}(h(r))$ whose initial and final endpoints in the clockwise direction along $\tilde{h}$ or $(h(r))$ are $\widetilde{z}_{i}^{+}(r)$ and $\widetilde{z}_{i+1}^{-}(r)$, the preimages of $z_{i}^{+}(r)$ and $z_{i+1}^{-}(r)$ (see Figure 8). Similarly, $\operatorname{ver}_{h(r)}^{+}\left(x_{i}(r)\right)$ and $\operatorname{ver}_{h(r)}^{-}\left(x_{i+1}(r)\right)$ have well-defined preimage $\operatorname{arcs} \operatorname{ver}_{h(r)}\left(\tilde{x}_{i}^{+}(r)\right)$ and $\widetilde{v e r}_{h(r)}\left(\tilde{x}_{i+1}^{-}(r)\right)$, with endpoints $\tilde{x}_{i}^{+}(r)$ and $\tilde{x}_{i+1}^{-}(r)$ in $\partial P$. Write $\widetilde{\Delta}_{i}(r)$ for the union $\widetilde{\Lambda}_{i}(r) \cup \tilde{\operatorname{ver}}_{h(r)}\left(\tilde{x}_{i}^{+}(r)\right) \cup \tilde{\operatorname{ver}}_{h(r)}\left(\tilde{x}_{i+1}^{-}(r)\right)$, so that

$$
\pi\left(\bigcup_{i=0}^{n-1} \widetilde{\Delta}_{i}(r)\right)=\partial D(q ; r) .
$$

Suppose that $\tilde{x}_{i+1}^{-}(r) \in \tilde{e}_{k}$ and $\tilde{x}_{i}^{+}(r) \in \tilde{e}_{\ell}$. The arc $\tilde{L}_{i}(r)$ of $\partial P$ from $\tilde{x}_{i+1}^{-}(r)$ to $\tilde{x}_{i}^{+}(r)$ in the counterclockwise direction therefore contains the sides $\tilde{e}_{k+1}, \ldots, \tilde{e}_{\ell-1}$, 
and segments of the sides $\tilde{e}_{k}$ and $\tilde{e}_{\ell}$. The polytrapezoid (union of trapezoids of the same height glued along their vertical sides) $\widetilde{T}_{i}(q ; r)$ bounded by $\widetilde{L}_{i}(r)$ and $\widetilde{\Delta}_{i}(r)$ satisfies $\pi\left(\widetilde{T}_{i}(q ; r)\right)=T_{i}(q ; r)$, and $\pi: \widetilde{T}_{i}(q ; r) \rightarrow T_{i}(q ; r)$ is a homeomorphism away from $\widetilde{L}_{i}(r)$. Notice that

$$
\bigcup_{i=0}^{n-1} \widetilde{L}_{i}(r)=\pi^{-1}\left(\bar{B}_{G}(q ; r)\right)
$$

and hence

$$
\sum_{i=0}^{n-1}\left|\tilde{L}_{i}(r)\right|=\mathrm{m}_{G}\left(\bar{B}_{G}(q ; r)\right)=m(q ; r) .
$$

Denote by $\theta_{0}(r), \theta_{1}(r)$ the internal angles of the polytrapezoid at the vertices $\tilde{x}_{i+1}^{-}(r)$, $\tilde{x}_{i}^{+}(r)$ respectively (the other internal angles along $\tilde{L}_{i}(r)$ are $2 \tilde{\theta}_{k+1}, \ldots, 2 \tilde{\theta}_{\ell}$, independent of $\left.r \in\left(r_{1}, r_{2}\right)\right)$.

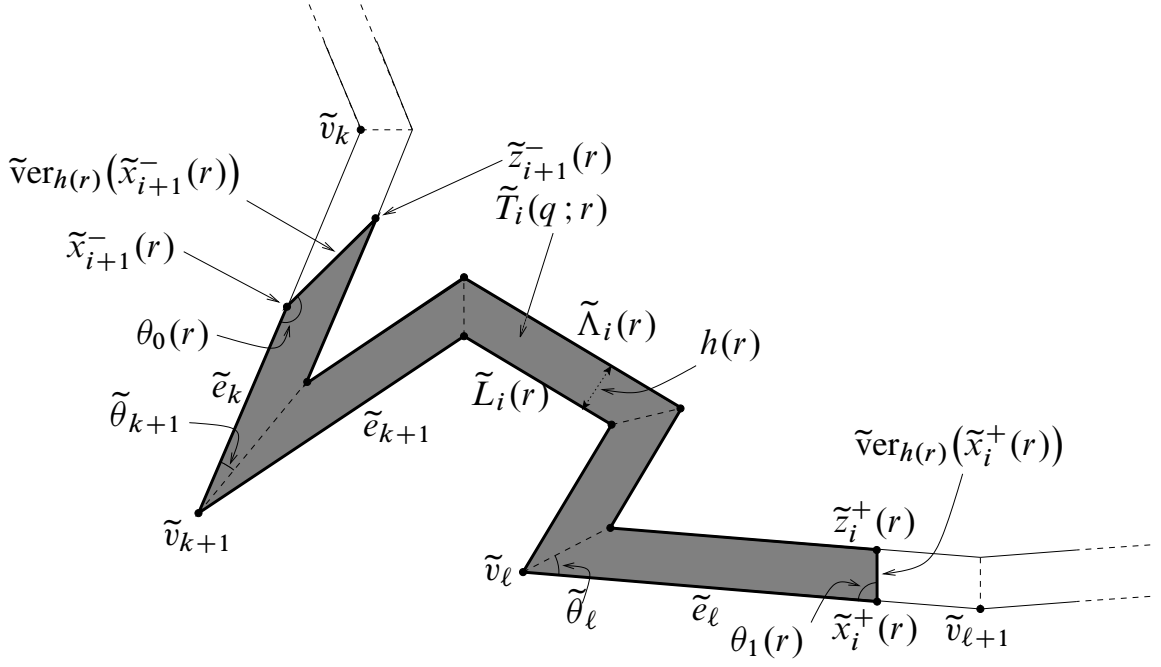

Figure 8: The polytrapezoid $\widetilde{T}_{i}(q ; r)$

\section{Lemma 71}

$$
\operatorname{Area}\left(\widetilde{T}_{i}(q ; r)\right)=h(r)\left|\widetilde{L}_{i}(r)\right|-\frac{h(r)^{2}}{2}\left(\cot \theta_{0}(r)+\cot \theta_{1}(r)+2 \sum_{j=k+1}^{\ell} \cot \tilde{\theta}_{j}\right) .
$$


Proof Observe that if a trapezoid has base $e$, top $e^{\prime}$, height $h$, and internal angles $\theta_{1}$ and $\theta_{2}$ at its base vertices, then

$$
\left|e^{\prime}\right|=|e|-h\left(\cot \theta_{1}+\cot \theta_{2}\right) .
$$

The result follows immediately on summing the areas $h\left(|e|+\left|e^{\prime}\right|\right) / 2$ of the constituent trapezoids of $\widetilde{T}_{i}(q ; r)$.

\section{Lemma 72}

$$
0<\frac{\mathrm{d}}{\mathrm{d} r} \operatorname{Area}\left(\widetilde{T}_{i}(q ; r)\right) \leq \frac{5 \bar{h}}{4 \bar{r}}\left(\left|\tilde{L}_{i}(r)\right|+r\right)
$$

Proof The first inequality in the statement is obvious.

Differentiating the expression for the area given by Lemma 71, recalling that $h(r)=$ $(\bar{h} / 2 \bar{r}) r$, and observing that $\frac{\mathrm{d}}{\mathrm{d} r}\left|\widetilde{L}_{i}(r)\right|=2$ and that

$$
\left|\tilde{\Lambda}_{i}(r)\right|=\left|\tilde{L}_{i}(r)\right|-h(r)\left(\cot \theta_{0}(r)+\cot \theta_{1}(r)+2 \sum_{j=k+1}^{\ell} \cot \tilde{\theta}_{j}\right)
$$

by (15), gives

$$
\begin{aligned}
\frac{\mathrm{d}}{\mathrm{d} r} \operatorname{Area}\left(\tilde{T}_{i}(q ; r)\right)= & \frac{\mathrm{d} h(r)}{\mathrm{d} r}\left[\left|\tilde{L}_{i}(r)\right|-h(r)\left(\cot \theta_{0}(r)+\cot \theta_{1}(r)+2 \sum_{j=k+1}^{\ell} \cot \tilde{\theta}_{j}\right)\right] \\
& +h(r)\left[\frac{\mathrm{d}}{\mathrm{d} r}\left|\tilde{L}_{i}(r)\right|-\frac{h(r)}{2} \frac{\mathrm{d}}{\mathrm{d} r}\left(\cot \theta_{0}(r)+\cot \theta_{1}(r)\right)\right] \\
\leq & \frac{\bar{h}}{2 \bar{r}}\left|\tilde{\Lambda}_{i}(r)\right|+\frac{\bar{h} r}{2 \bar{r}}\left(2+\frac{h(r)}{\bar{h}}\right) \\
\leq & \frac{\bar{h}}{\bar{r}}\left|\widetilde{L}_{i}(r)\right|+\frac{5 \bar{h}}{4 \bar{r}} r
\end{aligned}
$$

as required, using $\left|\widetilde{\Lambda}_{i}(r)\right| \leq 2\left|\tilde{L}_{i}(r)\right|$ (which follows from (11)) and Lemma 70 .

In order to estimate the $d_{S}$-distance between the boundary components of $\operatorname{Ann}(q ; r, s)$, where $r_{1}<r<s<r_{2}$, it is necessary to find a lower bound for the distance between $\widetilde{\Delta}_{i}(r)$ and $\widetilde{\Delta}_{i}(s)$, which project to part of the inner and the outer boundary components of $\operatorname{Ann}(q ; r, s)$ respectively.

Lemma 73 Let $\left(r_{1}, r_{2}\right)$ be an interval of $q$-planar radii. If $r_{1}<r<s<r_{2}$ then

$$
d\left(\widetilde{\Delta}_{i}(r), \widetilde{\Delta}_{i}(s)\right) \geq(s-r) \min \left\{\frac{\bar{h}}{2 \bar{r}}, \frac{1}{2}\right\} .
$$


Proof Let $\tilde{p} \in \widetilde{\Delta}_{i}(s)$. If $\tilde{p} \in \widetilde{\Lambda}_{i}(s)$, then every point of $\widetilde{\Delta}_{i}(r)$ is distance at least $h(s)-h(r)=\frac{\bar{h}}{2 \bar{r}}(s-r)$ from $\tilde{p}$.

If $\tilde{p} \in \tilde{v}^{-r_{h(s)}}\left(\tilde{x}_{i}^{+}(s)\right)$, then no point of $\tilde{\Delta}_{i}(r)$ can lie closer to $\tilde{p}$ than the closest point of $\tilde{\operatorname{ver}}_{h(s)}\left(\tilde{x}_{i}^{+}(r)\right)$ (the arc obtained by extending $\tilde{\operatorname{ver}}_{h(r)}\left(\tilde{x}_{i}^{+}(r)\right) \subset \widetilde{\Delta}_{i}(r)$ to hor $(h(s)))$. Hence $d\left(\tilde{p}, \widetilde{\Delta}_{i}(r)\right) \geq(s-r) / 2$ by (11). The proof if $\tilde{p} \in \tilde{\operatorname{ver}}_{h(s)}\left(\tilde{x}_{i+1}^{-}(s)\right)$ is similar.

\subsection{Proof of Theorem 59}

Initially in this section, $(P, \mathcal{P})$ is again a plain paper-folding scheme with associated paper sphere $S$ and scar $G \subset S$, and $q \in G$. The first step is to interpret the geometric results of the previous section in the sphere $S$.

Lemma 74 For all planar radii $r \in(0, \bar{r})$,

$$
0<\frac{\mathrm{d}}{\mathrm{d} r} \operatorname{Area}_{S}(D(q ; r)) \leq \frac{5 \bar{h}}{4 \bar{r}}(m(q ; r)+r \cdot n(q ; r)) .
$$

Proof Write $n=n(q ; r)$. From (13) it follows that

$$
\operatorname{Area}_{S}(D(q ; r))=\sum_{i=0}^{n-1} \operatorname{Area}_{\mathbb{R}^{2}}\left(\widetilde{T}_{i}(q ; r)\right) .
$$

Hence, by Lemma 72,

$$
\begin{aligned}
0<\frac{\mathrm{d}}{\mathrm{d} r} \operatorname{Area}_{S}(D(q ; r)) & \leq \frac{5 \bar{h}}{4 \bar{r}} \sum_{i=0}^{n-1}\left(\left|\tilde{L}_{i}(r)\right|+r\right) \\
& =\frac{5 \bar{h}}{4 \bar{r}}(m(q ; r)+r \cdot n(q ; r)),
\end{aligned}
$$

by (14) as required.

Lemma 75 Let $\left(r_{1}, r_{2}\right)$ be an interval of $q$-planar radii, and $r_{1}<r<s<r_{2}$. Let $\xi(r)$ and $\xi(s)$ denote the boundary components of $\operatorname{Ann}(q ; r, s)$. Then

$$
d_{S}(\xi(r), \xi(s)) \geq(s-r) \min \left\{\frac{\bar{h}}{2 \bar{r}}, \frac{1}{2}\right\} .
$$

Proof Let $D:=d_{S}(\xi(r), \xi(s))$, and let $p^{\prime} \in \xi(r)$ and $q^{\prime} \in \xi(s)$ be points with $d_{S}\left(p^{\prime}, q^{\prime}\right)=D$. Since $d_{S}$ is strictly intrinsic by Theorem 56(b), there is an arc $\gamma^{\prime}$ 
from $p^{\prime}$ to $q^{\prime}$ of length $D$, and apart from its endpoints this arc, being the shortest from $\xi(r)$ to $\xi(s)$, must lie in $\operatorname{Ann}(q ; r, s)$.

It will be shown that, for any $r \leq u<v \leq s$, the length of any arc $\gamma$ from $\xi(u)$ to $\xi(v)$ with $\stackrel{\gamma}{ } \subset \operatorname{Ann}(q ; u, v)$ is at least $C(v-u)$, where $C=\min \left\{\frac{\bar{h}}{2 \bar{r}}, \frac{1}{2}\right\}$, which will establish the result.

It can be assumed without loss of generality that there are no subarcs of $\gamma$ whose endpoints lie on the same component of $G \cap \overline{\operatorname{Ann}(q ; u, v)}$ and whose interiors lie entirely in the interior of a single $T_{i}(q ; v)$, since such a subarc could be replaced with a shorter subarc lying in $G$. Therefore the intersection of $\stackrel{\circ}{\gamma}$ with $G$ has a finite number $N$ of components. The result will be shown by induction on $N$.

If $N=0$, then the arc lies in a single $T_{i}(q ; v)$, and the result is immediate by Lemma 73 . If $N>0$, then let $t_{1}=\inf \{t>0: \gamma(t) \in G\}$ and $t_{2}=\inf \left\{t>t_{1}: \gamma(t) \notin G\right\}$, so that $\gamma\left(\left[t_{1}, t_{2}\right]\right) \subset G$. Split $\gamma$ into three subarcs $\gamma_{1}:\left[0, t_{1}\right] \rightarrow S, \gamma_{2}:\left[t_{1}, t_{2}\right] \rightarrow S$, and $\gamma_{3}:\left[t_{2}, 1\right] \rightarrow S$ (one of these arcs may degenerate to a point). Let $d\left(q, \gamma\left(t_{1}\right)\right)=w_{1} \in$ $[u, v]$ and $d\left(q, \gamma\left(t_{2}\right)\right)=w_{2} \in[u, v]$. By the inductive hypothesis, $\gamma_{1}$ has length at least $C\left(w_{1}-u\right)$ and $\gamma_{3}$ has length at least $C\left(v-w_{2}\right)$, while $\gamma_{2}$ lies entirely in a single $T_{i}\left(q ; w_{2}\right)$ and hence has length at least $C\left(w_{2}-w_{1}\right)$ by Lemma 73.

Lemma 76 Let $r$ and $s$ be $q$-planar radii with $0<r<s<\bar{r}$. Then

$$
\bmod \operatorname{Ann}(q ; r, s) \geq \int_{r}^{s} \frac{C^{2} \mathrm{~d} u}{\operatorname{Area}_{S}^{\prime}(D(q ; u))}
$$

where

$$
C:=\min \left\{\frac{\bar{h}}{2 \bar{r}}, \frac{1}{2}\right\} \quad \text { and } \quad \operatorname{Area}_{S}^{\prime}(D(q ; r)):=\frac{\mathrm{d} \mathrm{Area}_{S}(D(q ; r))}{\mathrm{d} r} .
$$

Proof Let $\left[r_{1}, r_{2}\right]$ be an interval of $q$-planar radii. Then

$$
\bmod \operatorname{Ann}\left(q ; r_{1}, r_{2}\right) \geq \frac{C^{2}\left(r_{2}-r_{1}\right)^{2}}{\operatorname{Area}_{S}\left(\operatorname{Ann}\left(q ; r_{1}, r_{2}\right)\right)}
$$

by (3) and Lemma 75.

For any partition $r_{1}=s_{0}<s_{1}<\cdots<s_{k}=r_{2}$ of $\left[r_{1}, r_{2}\right]$, it follows from (2) that

$$
\begin{aligned}
\bmod \operatorname{Ann}\left(q ; r_{1}, r_{2}\right) & \geq \sum_{j=1}^{k} \bmod \operatorname{Ann}\left(q ; s_{j-1}, s_{j}\right) \\
& \geq C^{2} \sum_{j=1}^{k} \frac{\left(s_{j}-s_{j-1}\right)^{2}}{\operatorname{Area}_{S}\left(D\left(q ; s_{j}\right)\right)-\operatorname{Area}_{S}\left(D\left(q ; s_{j-1}\right)\right)},
\end{aligned}
$$


and taking the supremum over all partitions of $\left[r_{1}, r_{2}\right]$ gives

$$
\bmod \operatorname{Ann}\left(q ; r_{1}, r_{2}\right) \geq \int_{r_{1}}^{r_{2}} \frac{C^{2} \mathrm{~d} u}{\operatorname{Area}_{S}^{\prime}(D(q ; u))},
$$

since $\operatorname{Area}_{S}^{\prime}(D(q ; u))$ is continuous and positive at planar radii. Hence for any collection of disjoint intervals $\left[r_{1}^{k}, r_{2}^{k}\right] \subset[r, s]$ of $q$-planar radii, (2) gives

$$
\bmod \operatorname{Ann}(q ; r, s) \geq \sum_{k} \int_{r_{1}^{k}}^{r_{2}^{k}} \frac{C^{2} \mathrm{~d} u}{\operatorname{Area}_{S}^{\prime}(D(q ; u))} .
$$

Taking the supremum over such collections of disjoint intervals, using the fact that the set of non-planar radii is closed and has zero measure, gives the result.

Combining the results of Lemmas 76 and 74 gives that, for all $q$-planar radii $0<r<$ $s<\bar{r}$,

$$
\bmod \operatorname{Ann}(q ; r, s) \geq \int_{r}^{s} \frac{C^{2} \mathrm{~d} u}{\frac{5 \bar{h}}{4 \bar{r}}(m(q ; r)+r \cdot n(q ; r))},
$$

where $C=\min \left\{\frac{\bar{h}}{2 \bar{r}}, \frac{1}{2}\right\}$. This motivates the following definition:

Definition 77 (Paper-folding goodness function) Let $(P, \mathcal{P})$ be a surface paper folding scheme, and let $P$ have polygon constants $\bar{h}$ and $\bar{r}$ (and $|\partial P|$ ). Let

$$
M=\frac{1}{5} \min \left\{\frac{\bar{r}}{\bar{h}}, \frac{\bar{h}}{\bar{r}}\right\},
$$

and define a function $\iota: G \times(0, \bar{r}) \rightarrow[0, \infty)$ by

$$
\iota(q ; r):= \begin{cases}\frac{M}{m(q ; r)+r \cdot n(q ; r)} & \text { if } n(q ; r)<\infty, \\ 0 & \text { otherwise. }\end{cases}
$$

$\iota$ is called a paper-folding goodness function for $(P, \mathcal{P})$.

Remarks 78 If $r$ is a $q$-planar radius then $\iota$ is non-zero and continuous at $(q ; r)$ by Lemma 58 (it is clear that $m(q ; r)$ is continuous at such a $(q ; r))$. Hence, using Lemma 58 again, for each $q \in G$ the set of radii $r$ at which $\iota(q ; r)=0$ or $\iota(q ; r)$ is discontinuous has measure zero.

As mentioned before, goodness functions are not uniquely determined since there is some freedom in the choice of polygon constants. What is important about them in this section is that their integrals down to zero be divergent. The rate of divergence will be important in Section 6, where moduli of continuity are discussed. 
Inequality (16) now becomes:

$$
\bmod \operatorname{Ann}(q ; r, s) \geq \int_{r}^{s} \iota(q ; t) \mathrm{d} t
$$

for every pair of planar radii $0<r<s<\bar{r}$.

Proof of Theorem 59 Let $q \in G$ be an isolated singular point. Pick a $q$-planar radius $r_{0} \in(0, \bar{r})$ small enough that $q$ is the only singular point in $D\left(q ; r_{0}\right)$, and let $W=D\left(q ; r_{0}\right) \backslash\{q\}$. Then $W$ is a Riemann surface, since it is contained in $S \backslash \mathcal{V}^{s}$ where there is a well-defined conformal structure. Since $W$ is topologically a disk minus a point, it is conformally homeomorphic to a plane domain (see Ahlfors-Sario [2], for example). In order to prove that $q$ is a puncture of the complex structure on $W$, observe that (17) yields, for $q$-planar radii $0<r<r_{0}$,

$$
\bmod \operatorname{Ann}\left(q ; r, r_{0}\right) \geq \int_{r}^{r_{0}} \iota(q ; s) \mathrm{d} s .
$$

Since this integral diverges as $r \searrow 0$ by hypothesis, the result follows from Lemma 22 and Riemann's Removable Singularity Theorem.

Only minor modifications are needed to treat the case of non-plain surface paper-folding schemes $(P, \mathcal{P})$. The constant $\bar{h}$ should be chosen in such a way that foliated collars of height $\bar{h}$ can be constructed for all of the polygons $P$, and the constant $\bar{r}$ should be chosen to be the injectivity radius of $G$. It then follows from Lemma 52 that $\bar{B}_{G}(q ; r)$ is a dendrite for all $q \in G$ and all $r<\bar{r}$, and the constructions and proof go through exactly as in the plain case.

\section{Modulus of continuity}

Let $(P, \mathcal{P})$ be a surface paper-folding scheme with associated paper surface $S$ and scar $G$. Suppose that the hypothesis of Theorem 59 is satisfied at some point $q \in G$ other than a non-isolated singularity (as explained in Remark 60, the hypothesis is necessarily satisfied when $q$ is a planar point or regular vertex). Then for any sufficiently small $q$-planar radius $r, D=\operatorname{Int}(D(q ; r))$ is a conformal disk, and by the uniformisation Theorem there is a conformal isomorphism $u: D \rightarrow \mathbb{D}$ to the unit disk $\mathbb{D} \subset \mathbb{C}$. In this section a modulus of continuity for $u$ is obtained.

Definition 79 (Modulus of continuity) Let $\rho:[0, \delta) \rightarrow[0, \infty)$, for some $\delta>0$, be a continuous and strictly increasing function for which $\rho(0)=0$. A function 
$f:\left(X, d_{X}\right) \rightarrow\left(Y, d_{Y}\right)$ between metric spaces has modulus of continuity $\rho$ at $x_{0} \in X$ if, for every $x \in X$ with $d_{X}\left(x_{0}, x\right)<\delta$,

$$
d_{Y}\left(f\left(x_{0}\right), f(x)\right) \leq \rho\left(d_{X}\left(x_{0}, x\right)\right) .
$$

$f$ is said to have modulus of continuity $\rho$ if the inequality above holds for every $x_{0} \in X$.

Remark 80 If $\mathcal{F}$ is a family of functions all of whose members have the same modulus of continuity, then $\mathcal{F}$ is uniformly equicontinuous: for all $\varepsilon>0$ there exists $\eta>0$ such that if two points are at distance less than $\eta$ then their images under any function in $\mathcal{F}$ (whose domain contains the two points) are at distance less than $\varepsilon$. Notice that there is no requirement for the domains of the functions in $\mathcal{F}$ to coincide: indeed, in the extreme case they may have empty intersection.

\subsection{Modulus of continuity at a point}

Here a local version of the main result of this section is presented: it provides an illustration of the ideas of the proof without the technical details which obscure the argument in the global case.

Let $(P, \mathcal{P})$ be a surface paper-folding scheme, $q$ be an isolated singularity in the scar $G$, and suppose that the hypothesis of Theorem 59 is satisfied at $q$, so that the complex structure on $S \backslash \mathcal{V}^{s}$ extends uniquely across $q$. It will be shown how it is possible to obtain a modulus of continuity for a uniformising map $u$ from a disk neighborhood of $q$ to the unit disk in the complex plane.

Pick a $q$-planar radius $r_{0} \in(0, \bar{r})$ small enough that $D:=\operatorname{Int}\left(D\left(q ; r_{0}\right)\right)$ contains no singularities other than $q$. Let $u: D \rightarrow \mathbb{D}$ be a conformal uniformising chart, where $\mathbb{D}$ is the open unit disk in the complex plane: normalise $u$ so that $u(q)=0$.

It is shown in Lemma 86 below that there are numbers $\delta>0$ and $A>0$, depending only on the polygon constants of $P$, such that

$$
B_{S}(q ; r) \subset D(q ; A r) \quad \text { for all } r \leq \delta .
$$

Pick any $x \in D$ with $d_{S}(q, x)<\min \left\{\delta, r_{0} / A\right\}$, and write $R:=A d_{S}(q, x)<r_{0}$, so that $x \in D(q ; R)$. Assume that $R$ is a $q$-planar radius (if not, increase it by an arbitrarily small amount). Then $\operatorname{Ann}\left(q ; R, r_{0}\right)$ separates $q$ and $x$ from $\partial D\left(q ; r_{0}\right)$, and hence $u\left(\operatorname{Ann}\left(q ; R, r_{0}\right)\right)$ separates $0=u(q)$ and $u(x)$ from the unit circle in $\mathbb{C}$. 
It follows from (17) and the Grötzsch annulus theorem (Theorem 25) that

$$
\begin{aligned}
\int_{R}^{r_{0}} \iota(q ; t) \mathrm{d} t & \leq \bmod \operatorname{Ann}\left(q ; R, r_{0}\right) \\
& =\bmod u\left(\operatorname{Ann}\left(q ; R, r_{0}\right)\right) \\
& \leq \frac{1}{2 \pi} \ln \frac{4}{|u(x)|} .
\end{aligned}
$$

Hence, recalling that $R=A d_{S}(q, x)$ (or $R$ is greater than $A d_{S}(q, x)$ by an arbitrarily small amount in the case where $R$ is not $q$-planar),

$$
|u(x)| \leq \frac{4}{\exp \left(2 \pi \int_{A d_{S}(q, x)}^{r_{0}} \iota(q ; t) \mathrm{d} t\right)},
$$

which is the desired modulus of continuity.

Remark 81 Since the projection $\pi: P \rightarrow S$ is distance non-increasing, a modulus of continuity $\rho_{q}$ for $u$ at $q$ is also a modulus of continuity for the composition $\phi:=u \circ \pi$ at the points of $\pi^{-1}(q)$ : if $q=\pi(\widetilde{q})$ and $x=\pi(\tilde{x})$, then

$$
\begin{aligned}
|\phi(\widetilde{q})-\phi(\tilde{x})| & =|u(q)-u(x)| \\
& \leq \rho_{q}\left(d_{S}(q, x)\right) \\
& \leq \rho_{q}\left(d_{P}(\widetilde{q}, \tilde{x})\right),
\end{aligned}
$$

since $d_{S}(q, x) \leq d_{P}(\tilde{q}, \tilde{x})$ and $\rho_{q}$ is increasing.

Example 82 Consider Examples 35 and 63 and suppose as in the second case of Example 63 that the fold lengths $a_{n}$ satisfy $a_{n} \asymp 1 / \lambda^{n}$ for some $\lambda>1$. As shown in that example, the hypothesis of Theorem 59 holds at the unique singularity $q_{0} \in G$, and hence the paper surface $S$ is conformally isomorphic to the Riemann sphere. Choose a uniformising map $u: S \rightarrow \widehat{\mathbb{C}}$ with $u\left(q_{0}\right)=0$, and let $\phi: P \rightarrow \widehat{\mathbb{C}}$ be the composition $\phi:=u \circ \pi$. Thus $\phi(0)=0$ : an explicit modulus of continuity for $\phi$ at 0 will now be found.

From the calculations of Example 63, a paper-folding goodness function at $q_{0}$ can be taken to be

$$
\iota\left(q_{0} ; r\right)=\frac{1}{C r \ln \frac{1}{r}},
$$

for some positive constant $C$, so that

$$
\int_{R}^{r_{0}} \iota\left(q_{0} ; t\right) \mathrm{d} t=\frac{1}{C}\left(\ln \ln \frac{1}{R}-\ln \ln \frac{1}{r_{0}}\right) .
$$


Choose $r_{0}$ small enough that $u\left(D\left(q ; r_{0}\right)\right) \subset \mathbb{D}$. Substitution in (18) yields, for $\tilde{x}$ close to 0 (how close depends on the choice of uniformising map $u$ )

$$
|\phi(\tilde{x})| \leq 4\left[\frac{\ln \left(1 / r_{0}\right)}{\ln (1 /(A|\tilde{x}|))}\right]^{2 \pi / C} .
$$

\subsection{Global modulus of continuity}

In the remainder of Section 6, the following assumptions will be made:

a) $(P, \mathcal{P})$ is a plain paper-folding scheme;

b) there are only finitely many singular points in the scar $G$; and

c) at each of these singular points, the hypothesis of Theorem 59 is satisfied.

It will be shown how to obtain a global modulus of continuity for a suitably normalised uniformising map $u: S \rightarrow \widehat{\mathbb{C}}$ from the paper sphere $S$ to the Riemann sphere (and hence also for the composition $\phi=u \circ \pi: P \rightarrow \widehat{\mathbb{C}}$ ).

Let $\iota(q ; r)$ be a paper-folding goodness function for $(P, \mathcal{P})$. Then

$$
\int_{0} \iota(q ; t) \mathrm{d} t=\infty
$$

for every $q \in G$ : at singular points this is the assumption (c) above, while at other points the integral diverges as explained in Remark 60.

Recall (Definition 68) that the polygon constants of $P$ are the numbers $\bar{h}, \bar{r}$, and $|\partial P|$ : here $\bar{h}$ is the height of the collaring of Section 5.3, and, since the paper-folding is plain, $\bar{r}>0$ can be chosen arbitrarily. A sensible choice is $\bar{r}=\bar{h}$, which maximises the constant $M$ in the goodness function $\iota$, with $M=1 / 5$.

For each $h \in(0, \bar{h}]$, write

$$
P_{h}:=P \backslash \widetilde{Q}(h),
$$

an open disk in $P \subset \mathbb{C}$ which is the complement of the collaring of height $h$.

To fix a uniformising map $u: S \rightarrow \widehat{\mathbb{C}}$, pick points $\tilde{p}_{0} \in \partial P$ and $\tilde{p}_{\infty} \in P_{\bar{h}}$. Set $p_{0}:=\pi\left(\widetilde{p}_{0}\right) \in G$ and $p_{\infty}:=\pi\left(\tilde{p}_{\infty}\right) \in S \backslash Q(\bar{h})$, and define $u: S \rightarrow \widehat{\mathbb{C}}$ to be the isomorphism with the following normalisation:

- $u\left(p_{0}\right)=0$

- $u\left(p_{\infty}\right)=\infty$; and

- the reciprocal $\Phi=1 / \phi$ of the composition $\phi:=u \circ \pi: P \rightarrow \widehat{\mathbb{C}}$ satisfies $\Phi^{\prime}\left(\tilde{p}_{\infty}\right)=1$. 
Observe that $\phi$ is injective and meromorphic in $\operatorname{Int}(P)$ with a simple pole at $\tilde{p}_{\infty}$.

Lemma 83 There is a constant $R=R(\bar{h})>0$, depending only on $\bar{h}$, such that

$$
\phi(\widetilde{Q}(\bar{h} / 2)) \subset \mathbb{D}_{R}:=\{z \in \mathbb{C}:|z|<R\}
$$

(or, equivalently, $\left.u(Q(\bar{h} / 2)) \subset \mathbb{D}_{R}\right)$.

Proof Since it lies in $P_{\bar{h}}$, the point $\tilde{p}_{\infty}$ is distance at least $\bar{h} / 2$ from any point of $\widetilde{Q}(\bar{h} / 2)$ : that is, $\widetilde{Q}(\bar{h} / 2) \subset P \backslash B_{\mathbb{C}}\left(\tilde{p}_{\infty}, \bar{h} / 2\right)$. Therefore

$$
\Phi(\tilde{Q}(\bar{h} / 2)) \subset \Phi\left(P \backslash B_{\mathbb{C}}\left(\tilde{p}_{\infty} ; \bar{h} / 2\right)\right)=\widehat{\mathbb{C}} \backslash \Phi\left(B_{\mathbb{C}}\left(\tilde{p}_{\infty} ; \bar{h} / 2\right)\right),
$$

where $\Phi=1 / \phi$. Since $\Phi$ is univalent in $B_{\mathbb{C}}\left(\tilde{p}_{\infty} ; \bar{h} / 2\right)$ with $\Phi\left(\tilde{p}_{\infty}\right)=0$ and $\Phi^{\prime}\left(\tilde{p}_{\infty}\right)=1$, Koebe's one-quarter theorem gives a radius $s(\bar{h})>0$ such that

$$
\Phi\left(B_{\mathbb{C}}\left(\tilde{p}_{\infty} ; \bar{h} / 2\right)\right) \supset \overline{\mathbb{D}}_{s(\bar{h})},
$$

and the result follows with $R(\bar{h})=1 / s(\bar{h})$.

The main theorem which will be proved in this section is:

Theorem 84 Let $(P, \mathcal{P})$ be a plain paper-folding scheme with only finitely many singular points in its scar $G$, at each of which the conditions of Theorem 59 hold. Then the uniformising map $\phi: P \rightarrow \widehat{\mathbb{C}}$ has a modulus of continuity $\bar{\rho}$, with respect to the Euclidean metric on $P$ and the spherical metric on $\widehat{\mathbb{C}}$, which depends only on the polygon constants of $P$ and on the paper-folding goodness function $\iota: G \times(0, \bar{r}) \rightarrow$ $[0, \infty)$.

Recall that the spherical metric $d_{\widehat{\mathbb{C}}}$ on $\widehat{\mathbb{C}}$ is defined by

$$
d_{\widehat{\mathbb{C}}}(w, z)=\inf _{\gamma \in \Gamma}\left(2 \int_{\gamma} \frac{|\mathrm{d} z|}{1+|z|^{2}}\right)
$$

where $\Gamma$ is the set of paths in $\widehat{\mathbb{C}}$ from $w$ to $z$. The only properties of the spherical metric which will be used here are: $d_{\widehat{\mathbb{C}}}(1 / w, 1 / z)=d_{\widehat{\mathbb{C}}}(w, z)$ for all $w, z \in \widehat{\mathbb{C}}$; and $d_{\widehat{\mathbb{C}}}(w, z) \leq 2|w-z|$ for all $w, z \in \mathbb{C}$. 


\subsection{Two technical lemmas}

Recall from Section 5.3.2 that $\tilde{\psi}_{\bar{h}}: \widetilde{Q}(\bar{h}) \rightarrow \partial P$ is the retraction obtained by sliding points along leaves of Ver: for economy of notation, this retraction will henceforth be denoted $\tilde{\psi}$, and similarly $\psi$ rather than $\psi_{\bar{h}}$ will be used to denote the retraction $\pi \circ \tilde{\psi} \circ \pi^{-1}: Q(\bar{h}) \rightarrow G$. The first lemma in this section essentially says that $\psi$ is Lipschitz with constant determined by the polygon constants.

Lemma 85 Let $\alpha$ be a rectifiable path in $Q(\bar{h})$. Then

$$
|\psi \circ \alpha|_{G} \leq \frac{|\partial P|}{\bar{h}}|\alpha|_{S}
$$

Sketch proof Lift $\alpha$ to a path in $\widetilde{Q}(\bar{h})$, and consider a point $\tilde{\gamma}(t, h)$ of $\alpha$ lying in a trapezoid $\widetilde{Q}_{i}$. As in the proof of Lemma 70, extend the vertical sides of $\widetilde{Q}_{i}$ to form a triangle $T$ of height $H \geq 2 \bar{h}$. The action of $\tilde{\psi}$ in $\widetilde{Q}_{i}$ is projection from the apex of $T$ onto the base of $\widetilde{Q}_{i}$.

An infinitesimal arc through $\tilde{\gamma}(t, h)$ is maximally stretched by $\tilde{\psi}$ when it is perpendicular to the line segment from $\tilde{\gamma}(t, h)$ to the apex of $T$, in which case it is stretched by a factor $\frac{H}{(H-h) \sin \tilde{\theta}(t)}$. The result follows using (12) and $H \geq 2 h$.

The following lemma relates metric balls $B_{S}(q, \delta)$ in $S$ to the disks $D(\psi(q) ; \mu)$ for $q$ in a thinner collar $Q(\delta)=\pi \circ \widetilde{Q}(\delta)$ (see Figure 9).

\section{Lemma 86 Let}

$$
\delta=\frac{1}{4} \min \left\{\bar{h}, \bar{r}, \frac{2 \bar{h} \bar{r}}{|\partial P|}\right\} \quad \text { and } \quad A=\frac{\bar{r}}{2 \delta}=2 \max \left\{\frac{\bar{r}}{\bar{h}}, 1, \frac{|\partial P|}{2 \bar{h}}\right\} .
$$

Then for every $q \in Q(\delta)$ and every $r \in[0, \delta]$,

$$
\bar{B}_{S}(q ; r) \subset D\left(\psi(q) ; A\left(r+h_{q}\right)\right),
$$

where $h_{q} \in[0, \delta]$ is the height of $q$ (that is $q \in \operatorname{hor}\left(h_{q}\right)$ ).

Remark 87 Notice that $A\left(r+h_{q}\right) \leq A(\delta+\delta)=\bar{r}$, so that $D\left(\psi(q) ; A\left(r+h_{q}\right)\right)$ is defined (even when $A\left(r+h_{q}\right)$ is not a $\psi(q)$-planar radius, in which case it may not be a disk).

Proof of Lemma 86 Observe first that the $d_{S}$-distance from $q$ to the boundary hor $(\bar{h})$ of $Q=Q(\bar{h})$ is at least $\bar{h}-h_{q} \geq \bar{h}-\delta \geq 3 \bar{h} / 4>\delta \geq r$, so that $\bar{B}_{S}(q ; r) \subset Q(\bar{h})$. 


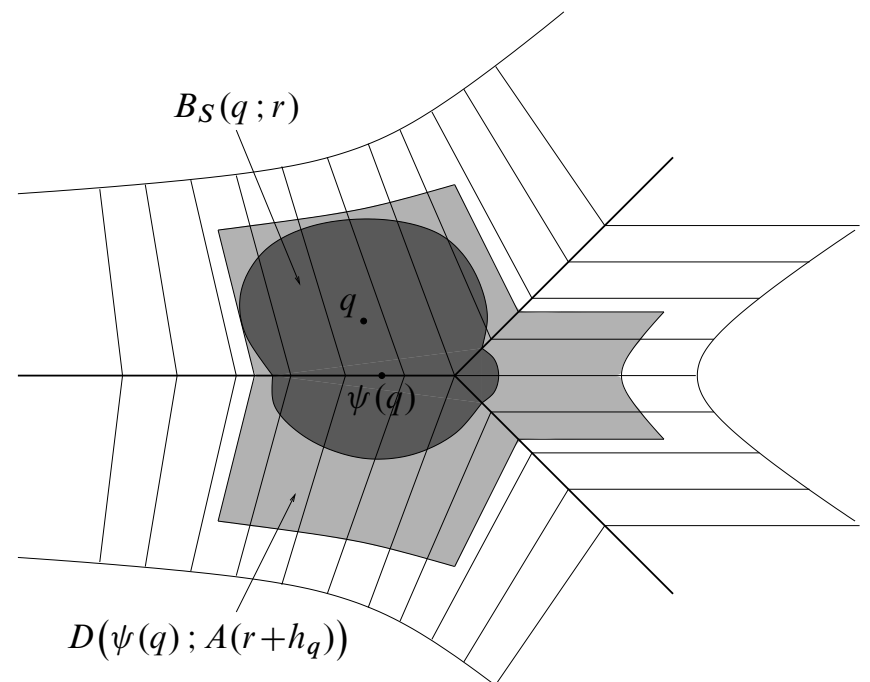

Figure 9: The disk $D\left(\psi(q) ; A\left(r+h_{q}\right)\right)$ and the ball $B_{S}(q ; r)$.

Write $K=A\left(r+h_{q}\right)$. Since $D(\psi(q) ; K)=\psi_{h(K)}^{-1}(\bar{B}(q ; K))$ where $h(K)=\bar{h} K / 2 \bar{r}$ (Definition 65), it is required to show that every point $x \in S$ with $d_{S}(q, x) \leq r$ has height $h_{x} \leq \bar{h} K / 2 \bar{r}$ and satisfies $d_{G}(\psi(q), \psi(x)) \leq K$.

The former property is immediate since $h_{x} \leq r+h_{q}=K / A \leq \bar{h} K / 2 \bar{r}$. For the latter, since $d_{S}$ is strictly intrinsic (Theorem 56(b)), there is a path in $S$ from $q$ to $x$ of length $d_{S}(q, x)$, and this path must lie in $Q(\bar{h})$ since the distance from $q$ to hor $(\bar{h})$ is greater than $r$. The image under $\psi$ of this path is a path in $G$ from $\psi(q)$ to $\psi(x)$, whose length is no more than $\frac{|\partial P|}{\bar{h}} d_{S}(q, x)$ by Lemma 85 . Hence

$$
d_{G}(\psi(q), \psi(x)) \leq \frac{|\partial P|}{\bar{h}} r \leq A\left(r+h_{q}\right)=K
$$

as required.

\subsection{Modulus of continuity in a collar}

Let $\delta$ and $A$ be given by (20), and $R=R(\bar{h})>0$ be the constant given by Lemma 83 . Define functions $\mu: Q(\delta) \times[0, \delta) \rightarrow[0, \bar{r})$ and $\rho: Q(\delta) \times[0, \delta) \rightarrow[0, \infty)$ by

$$
\mu(q, t):=A\left(t+h_{q}\right)
$$


(so $\mu(q, t)<2 A \delta=\bar{r}$ ) and

$$
\rho_{q}(t)=\rho(q, t):= \begin{cases}0, & \text { if } t=0 ; \\ \frac{8 R t}{h_{q} \cdot \exp \left(2 \pi \int_{\mu\left(q, h_{q}\right)}^{\bar{r}} \iota(\psi(q) ; s) \mathrm{d} s\right)}, & \text { if } 0<t \leq h_{q} ; \\ \frac{8 R}{\exp \left(2 \pi \int_{\mu(q, t)}^{\bar{r}} \iota(\psi(q) ; s) \mathrm{d} s\right)}, & \text { if } h_{q} \leq t \leq \delta .\end{cases}
$$

It will be shown in this section that, for every $\widetilde{q} \in \widetilde{Q}(\delta), \rho_{\pi(\widetilde{q})}$ is a modulus of continuity for $\phi: P \rightarrow \widehat{\mathbb{C}}$ at $\widetilde{q}$.

Remark 88 For each $q \in Q(\delta), \rho_{q}:[0, \delta) \rightarrow[0, \infty)$ is a modulus of continuity in the sense of Definition 79:

(a) It is continuous in $(0, \delta)$ and strictly increasing since:

- when $t \in\left(0, h_{q}\right], \rho_{q}(t)$ is proportional to $t$;

- when $t \in\left(h_{q}, \delta\right), \rho_{q}(t)$ is inversely proportional to

$$
\exp \left(2 \pi \int_{A\left(t+h_{q}\right)}^{\bar{r}} \iota(\psi(q) ; s) \mathrm{d} s\right),
$$

which depends continuously on $t$, and is strictly decreasing since $A\left(t+h_{q}\right)$ is strictly increasing and $\iota$ is positive except on a set of measure zero; and

- when $t=h_{q}>0$, the two expressions for $\rho_{q}$ clearly agree.

(b) It is continuous at 0 since

- If $q \in G$ (that is $h_{q}=0$ ), the second expression in (21) is used, and the integral in its denominator diverges as $t \rightarrow 0$ by assumption; and

- If $q \notin G$ (that is $h_{q}>0$ ), the first expression in (21) is used for $t$ sufficiently small, which clearly converges to 0 as $t \rightarrow 0$.

The (joint) continuity of $\rho: Q(\delta) \times[0, \delta) \rightarrow[0, \infty)$ will be proved in Lemma 92 below.

Example 89 For the simplest possible example, suppose that $q \in G$ is a planar point, and that $\bar{r}$ is small enough that $m(q ; r)=4 r$ and $n(q ; r)=2$ for all $r \leq \bar{r}$ : hence $\iota(q ; r)=M / 6 r$. Then $(21)$ gives a modulus of continuity

$$
\rho_{q}(t)=\frac{8 R}{\exp \left(\int_{A t}^{\bar{r}} \frac{\pi M}{3 s} \mathrm{~d} s\right)}=8 R\left(\frac{A}{\bar{r}}\right)^{\frac{\pi M}{3}} t^{\frac{\pi M}{3}} .
$$


Since $M \leq 1 / 5$ (Definition 77), the exponent $\pi M / 3$ lies in $(0,1)$. This modulus of continuity is of course not optimal, since the uniformising map is Lipschitz near planar points.

Lemma 90 If $\widetilde{q} \in \widetilde{Q}(\delta)$, then $\phi: P \rightarrow \widehat{\mathbb{C}}$ has modulus of continuity $\rho_{\pi(\widetilde{q})}$ at $\widetilde{q}$. That is, if $\widetilde{q} \in \tilde{Q}(\delta)$ and $\tilde{x} \in P$ with $d_{P}(\widetilde{q}, \tilde{x})<\delta$, then

$$
|\phi(\widetilde{q})-\phi(\tilde{x})| \leq \rho_{\pi(\widetilde{q})}\left(d_{P}(\tilde{q}, \tilde{x})\right) .
$$

Proof Notice that $\phi(\widetilde{q})$ and $\phi(\tilde{x})$ lie in $\mathbb{C}$ (since $\phi^{-1}(\infty)=\tilde{p}_{\infty} \notin \widetilde{Q}(\bar{h})$ ), and so $|\phi(\widetilde{q})-\phi(\tilde{x})|$ makes sense.

As explained in Remark 81, a modulus of continuity for $u: S \rightarrow \widehat{\mathbb{C}}$ is also a modulus of continuity for $\phi=u \circ \pi$, and it therefore suffices to obtain the former. So let $q \in Q(\delta)$, and let $x \in S$ be such that

$$
t:=d_{S}(q, x)<\delta .
$$

It is required to show that $|u(q)-u(x)| \leq \rho_{q}(t)$.

Case $1\left(h_{q} \leq t\right) \quad x \in D(\psi(q) ; \mu(q, t))$ by Lemma 86. Assuming at first that $\mu(q, t)$ is a $\psi(q)$-planar radius, this means the annular region $\operatorname{Ann}(\psi(q) ; \mu(q, t), \bar{r})$ separates $q$ and $x$ from $S \backslash Q(\bar{h} / 2)$. Hence, by Lemma 83, the image $u(\operatorname{Ann}(\psi(q) ; \mu(q, t), \bar{r}))$ separates $u(q)$ and $u(x)$ from the circle $\{|z|=R\}$. The modulus of continuity can thus be obtained from the Grötzsch annulus theorem (Theorem 25) just as in Section 6.1:

$$
\begin{aligned}
\int_{\mu(q, t)}^{\bar{r}} \iota(\psi(q) ; s) \mathrm{d} s & \leq \bmod \operatorname{Ann}(\psi(q) ; \mu(q, t), \bar{r}) \\
& =\bmod u(\operatorname{Ann}(\psi(q) ; \mu(q, t), \bar{r})) \\
& \leq \bmod \operatorname{Gr}\left(\mid \frac{u(q)-u(x)}{\left.R-\frac{u(x) \overline{u(q)}}{R} \mid\right)}\right. \\
& \leq \bmod \operatorname{Gr}\left(\frac{|u(q)-u(x)|}{2 R}\right) \\
& \leq \frac{1}{2 \pi} \ln \frac{8 R}{|u(q)-u(x)|}
\end{aligned}
$$

(where the Möbius transformation

$$
z \mapsto \frac{R(z-u(q))}{R^{2}-z \overline{u(q)}}
$$


has been used to move $u(q)$ to 0 and the circle $|z|=R$ to the circle $|z|=1$ ), giving

$$
|u(q)-u(x)| \leq \frac{8 R}{\exp \left(2 \pi \int_{\mu(q, t)}^{\bar{r}} \iota(\psi(q) ; s) \mathrm{d} s\right)}
$$

as required.

If $\mu(q ; t)$ is not $\psi(q)$-planar, then increasing it by an arbitrarily small amount to a planar radius gives the result.

Case $2\left(0<t \leq h_{q}\right)$ Since $x \in D(\psi(q) ; \mu(q, t)) \subset D\left(\psi(q) ; \mu\left(q, h_{q}\right)\right)$, the annular region $\operatorname{Ann}\left(\psi(q) ; \mu\left(q, h_{q}\right), \bar{r}\right)$ separates $q$ and $x$ from $S \backslash Q(\bar{h} / 2)$ (if $\mu\left(q, h_{q}\right)$ is not a planar radius, then perturb as in Case 1$)$. Moreover, since $\bar{B}_{S}\left(q ; h_{q}\right) \subset$ $D\left(\psi(q) ; \mu\left(q, h_{q}\right)\right)$ by Lemma 86 , the annular region $B_{S}\left(q ; h_{q}\right) \backslash \bar{B}_{S}(q ; t)$ is nested inside the first annulus and also separates $q$ and $x$ from $S \backslash Q(\bar{h} / 2)$. Arguing as in Case 1, it follows that $\bmod \left(B_{S}\left(q ; h_{q}\right) \backslash \bar{B}_{S}(q ; t)\right)+\bmod \operatorname{Ann}\left(\psi(q) ; \mu\left(q, h_{q}\right), \bar{r}\right) \leq \frac{1}{2 \pi} \ln \frac{8 R}{|u(q)-u(x)|}$. Since $B_{S}\left(q ; h_{q}\right)$ is isometric to a plane metric ball,

$$
\bmod \left(B_{S}\left(q ; h_{q}\right) \backslash \bar{B}_{S}(q ; t)\right)=\frac{1}{2 \pi} \ln \frac{h_{q}}{t},
$$

and hence

$$
\frac{1}{2 \pi} \ln \frac{h_{q}}{t}+\int_{\mu\left(q, h_{q}\right)}^{\bar{r}} \iota(\psi(q) ; s) \mathrm{d} s \leq \frac{1}{2 \pi} \ln \frac{8 R}{|u(q)-u(x)|}
$$

so that

$$
|u(q)-u(x)| \leq \frac{8 R t}{h_{q} \cdot \exp \left(2 \pi \int_{\mu\left(q, h_{q}\right)}^{\bar{r}} \iota(\psi(q), s) \mathrm{d} s\right)}
$$

as required.

In order to use Lemma 90 to construct a global modulus of continuity in $Q(\delta)$, the maximum over the functions $\rho_{q}$ will be taken. To show that this maximum is itself continuous it is necessary to prove that $\rho: Q(\delta) \times[0, \delta] \rightarrow[0, \infty)$ is continuous: this is the aim of the remainder of this section.

Lemma 91 Let $(P, \mathcal{P})$ be a plain paper-folding scheme with only finitely many singular points in its scar $G$, at each of which the conditions of Theorem 59 hold. Define $I: G \times(0, \bar{r}) \rightarrow(0, \infty)$ by

$$
I(q, r)=\int_{r}^{\bar{r}} \iota(q ; s) \mathrm{d} s .
$$


Then

(a) I is continuous, and

(b) For all $K>0$, there is some $\eta>0$ such that $I(q, r)>K$ for all $q \in G$ and all $r \in(0, \eta)$.

Proof Recall that

$$
I(q, r)=\int_{r}^{\bar{r}} \iota(q ; s) \mathrm{d} s=M \int_{r}^{\bar{r}} \frac{\mathrm{d} s}{m(q ; s)+s \cdot n(q ; s)},
$$

where $m(q ; s)=\mathrm{m}_{G}\left(B_{G}(q ; s)\right)$.

(a) Let $\left(q_{0} ; r_{0}\right) \in G \times(0, \bar{r})$ : it will be shown that $I$ is continuous at $\left(q_{0} ; r_{0}\right)$. Now for $(q ; r) \in G \times(0, \bar{r})$,

$$
I(q, r)-I\left(q_{0}, r_{0}\right)=\int_{r_{0}}^{\bar{r}} \iota(q ; s) \mathrm{d} s-\int_{r_{0}}^{\bar{r}} \iota\left(q_{0} ; s\right) \mathrm{d} s+\int_{r}^{r_{0}} \iota(q ; s) \mathrm{d} s .
$$

The final integral converges to zero as $(q, r) \rightarrow\left(q_{0}, r_{0}\right)$, since $\iota(q ; s)$ is bounded above by $M / r_{0}$ for $s \geq r_{0} / 2$. Hence it suffices to prove that, for all $\varepsilon>0$,

$$
\begin{aligned}
\left|\int_{r_{0}}^{\bar{r}}\left(\iota(q ; s)-\iota\left(q_{0} ; s\right)\right) \mathrm{d} s\right| & = \\
M & \left|\int_{r_{0}}^{\bar{r}}\left(\frac{1}{m(q ; s)+s \cdot n(q ; s)}-\frac{1}{m\left(q_{0} ; s\right)+s \cdot n\left(q_{0} ; s\right)}\right)\right|<\varepsilon
\end{aligned}
$$

provided $d_{G}\left(q, q_{0}\right)$ is sufficiently small.

Because the absolute value of the integrand is bounded above by $M / r_{0}$, a small open interval of radius $\delta_{1}$ can be excised from the range of integration about each of the finitely many values of $s$ for which there is a singularity at distance $s$ from $q_{0}$, without changing the integral by more than $\varepsilon / 3$. Again, since there are now only finitely many values of $s$ in the range of integration for which there is a vertex at distance $s$ from $q_{0}$, further open intervals of radius $\delta_{2} \leq \delta_{1}$ can be excised about each of these values without changing the integral by more than an additional $\varepsilon / 3$.

Now if $d_{G}\left(q, q_{0}\right)<\delta_{2} / 2$ then $n\left(q_{0} ; s\right)=n(q ; s)$ in each of the remaining intervals of integration. Moreover, $m(q ; s)$ is continuous in both variables for $q \in B_{G}\left(q_{0} ; \delta_{2} / 2\right)$ and $s$ in a single interval of integration, and the result follows. 
(b) Let $q_{0} \in G$. Since (8) holds at $q_{0}$, there is some $\varepsilon>0$ such that

$$
M \int_{\varepsilon}^{\bar{r}} \frac{\mathrm{d} s}{m\left(q_{0} ; s\right)+s \cdot n\left(q_{0} ; s\right)}>3 K .
$$

The integrand is bounded above by $M / \varepsilon$ so, as in part (a), there is a subset $L$ of $[\varepsilon, \bar{r}]$ and a number $\delta>0$ such that:

- $L$ consists of finitely many intervals;

- $\left(d_{G}\left(q_{0}, q^{*}\right)-\delta, d_{G}\left(q_{0}, q^{*}\right)+\delta\right)$ is disjoint from $L$ for any non-planar point $q^{*}$ of $G$; and

- $\quad M \int_{L} \frac{\mathrm{d} s}{m\left(q_{0} ; s\right)+s \cdot n\left(q_{0} ; s\right)}>2 K$.

Now if $s \in L$ then $n\left(q_{0} ; s\right)=n(q ; s)$ for $d_{G}\left(q, q_{0}\right)<\delta / 2$; and $m$ is continuous in $B_{G}\left(q_{0} ; \delta / 2\right) \times L$. There is therefore some $\delta^{\prime} \in(0, \delta / 2)$ such that

$$
M \int_{L} \frac{\mathrm{d} s}{m(q ; s)+s \cdot n(q ; s)}>K
$$

provided that $d_{G}\left(q ; q_{0}\right)<\delta^{\prime}$. Hence

$$
I(q, r)>\int_{\varepsilon}^{\bar{r}} \iota(q ; s) \mathrm{d} s \geq \int_{L} \iota(q ; s) \mathrm{d} s>K
$$

for all $q$ with $d_{G}\left(q, q_{0}\right)<\delta^{\prime}$ and all $r \in(0, \varepsilon)$. The result follows by compactness of $G$.

Lemma 92 Let $(P, \mathcal{P})$ be a plain paper-folding scheme with only finitely many singular points in its scar $G$, at each of which the conditions of Theorem 59 hold. Then the function $\rho: Q(\delta) \times[0, \delta) \rightarrow[0, \infty)$ of $(21)$ is continuous.

Proof Observe that if $\xi: Q(\delta) \times(0, \delta) \rightarrow[1, \infty)$ is defined by

$$
\xi(q, t)=\max \left(1, \frac{h_{q}}{t}\right),
$$

then (21) can be written

$$
\rho(q, t)= \begin{cases}0, & \text { if } t=0 ; \\ \frac{8 R}{\xi(q, t) \cdot \exp \left(2 \pi \int_{\mu(q, t \xi(q, t))}^{\bar{r}} \iota(\psi(q) ; s) \mathrm{d} s\right)}, & \text { if } t>0 .\end{cases}
$$

Since $\xi, \mu$ and $\psi$ are continuous, the continuity of $\rho$ at points $\left(q_{0}, t_{0}\right)$ with $t_{0}>0$ follows from Lemma 91(a). 
It remains to prove that $\rho$ is continuous at all points $\left(q_{0}, 0\right)$ with $q_{0} \in Q(\delta)$ : that is, that $\rho(q, t) \rightarrow 0$ as $(q, t) \rightarrow\left(q_{0}, 0\right)$ with $t>0$.

If $h_{q_{0}}>0$ then $\xi(q, t)=h_{q} / t$ for all $(q, t)$ close to $\left(q_{0}, 0\right)$, so that $\xi(q, t) \rightarrow \infty$, and hence $\rho(q, t) \rightarrow 0$, as $(q, t) \rightarrow\left(q_{0}, 0\right)$.

If $h_{q_{0}}=0$ (that is $\left.q_{0} \in G\right)$ then, since $\xi(q, t) \geq 1$ for all $(q, t)$, the result is immediate from Lemma 91(b) and the continuity of $\psi$.

\subsection{Modulus of continuity in the interior}

In Section 6.4, a modulus of continuity for $\phi$ was obtained at points $\widetilde{q} \in \widetilde{Q}(\delta)$, where $\delta$ is given by (20). In this section, a modulus of continuity is derived at points $\widetilde{q}$ in the complement $P_{\delta / 2}$ of $\widetilde{Q}(\delta / 2)$. These overlapping moduli of continuity will then be glued together in Section 6.6 to give the required global modulus.

Lemma 93 Let $\tilde{q}, \tilde{x} \in P_{\delta / 2}$. Then

$$
d_{\widehat{\mathbb{C}}}(\phi(\widetilde{q}), \phi(\tilde{x})) \leq \kappa d_{P}(\tilde{q}, \tilde{x}),
$$

where

$$
\kappa=2 \exp \left(\frac{16 \operatorname{diam}_{P_{\delta / 2}} P_{\delta / 2}}{\delta}\right) \leq 2 \exp \left(\frac{32|\partial P|}{\delta}\right)
$$

(recall that $d_{\widehat{\mathbb{C}}}$ denotes the spherical metric on $\widehat{\mathbb{C}}$ ). That is, $\phi$ is Lipschitz in $P_{\delta / 2}$ with a constant which depends only on the polygon constants of $P$.

Proof Let $\Phi: \operatorname{Int}(P) \rightarrow \mathbb{C}$ be defined by $\Phi:=1 / \phi$. Then $\Phi$ is univalent with $\Phi\left(\tilde{p}_{\infty}\right)=0$ and $\Phi^{\prime}\left(\tilde{p}_{\infty}\right)=1$ by the choice of normalisation of $\phi$. It follows from Theorem 26 that $\Phi$ is Lipschitz in $P_{\delta / 2}$ with constant $\kappa / 2$, where $\kappa$ is as in the statement of the lemma. Then

$$
\begin{aligned}
d_{\widehat{\mathbb{C}}}(\phi(\widetilde{q}), \phi(\tilde{x})) & =d_{\widehat{\mathbb{C}}}(\Phi(\widetilde{q}), \Phi(\tilde{x})) \\
& \leq 2|\Phi(\widetilde{q})-\Phi(\tilde{x})| \\
& \leq \kappa d_{P}(\widetilde{q}, \tilde{x})
\end{aligned}
$$

as required. Finally, observe that

$$
\operatorname{diam}_{P_{\delta / 2}} P_{\delta / 2} \leq 2|\partial P|
$$

since the path between any two points of $P_{\delta / 2}$ obtained by connecting each with a horizontal arc to the boundary and then joining the endpoints of these arcs with the shorter boundary arc between them has length bounded above by $\frac{1}{2}|\partial P|+\frac{1}{2}|\partial P|+$ $\frac{1}{2}\left|\partial P_{\delta / 2}\right| \leq 2|\partial P|$ by $(11)$. 


\subsection{Proof of Theorem 84}

Define $\hat{\rho}:[0, \delta) \rightarrow(0, \infty)$ by

$$
\hat{\rho}(t):=2 \max _{q \in Q(\delta)} \rho(q, t),
$$

which is well defined since $\rho$ is continuous and $Q(\delta)$ is compact. Then $\hat{\rho}$ is a continuous strictly increasing function with $\hat{\rho}(0)=0$ (since each $\rho_{q}$ has these properties and $\rho$ is continuous), and $\phi$ has modulus of continuity $\hat{\rho}$ on $\widetilde{Q}(\delta)$ with respect to the Euclidean metric on $\widetilde{Q}(\delta)$ and the spherical metric on $\widehat{\mathbb{C}}$ (the factor 2 arises from the translation from the Euclidean to the spherical metric).

On the other hand, $\phi$ is $\kappa$-Lipschitz in $P_{\delta / 2}$ by Lemma 93, where $\kappa=\kappa(\delta,|\partial P|)$ is given by (22). Hence $\bar{\rho}:[0, \delta) \rightarrow[0, \infty)$ defined by

$$
\bar{\rho}(t):=\max \{\hat{\rho}(t), \kappa t\}
$$

is the desired modulus of continuity.

\section{A dynamical application: convergence to the tight horse- shoe}

\subsection{Introduction}

This section contains an extended example. It illustrates that it is practicable to estimate the modulus of continuity provided by (21) uniformly across a family of paper-folding schemes, and thereby to construct limits which are of interest in dynamical systems theory. The example is the simplest non-trivial one known to the authors, but the context in which it arises, which is described below, provides a wealth of similar cases. In fact, similar arguments construct an uncountable collection of limiting maps in the family of unimodal generalised pseudo-Anosov maps defined in de Carvalho-Hall [6] as will be shown elsewhere.

The family of tent maps $T_{\lambda}:[0,1] \rightarrow[0,1]$, where $\lambda \in(1,2]$, is defined by

$$
T_{\lambda}(x)= \begin{cases}\lambda(x-1)+2 & \text { if } x \leq 1-\frac{1}{\lambda} \\ \lambda(1-x) & \text { if } x \geq 1-\frac{1}{\lambda}\end{cases}
$$

Thus $T_{\lambda}(0)=2-\lambda, T_{\lambda}\left(1-\frac{1}{\lambda}\right)=1, T_{\lambda}(1)=0$, and $T_{\lambda}$ has constant slope $\lambda$ on $\left[0,1-\frac{1}{\lambda}\right]$ and constant slope $-\lambda$ on $\left[1-\frac{1}{\lambda}, 1\right]$ (see Figure 10).

Tent maps have been widely studied in the field of unimodal dynamics, not least because every unimodal map $f:[0,1] \rightarrow[0,1]$ with positive topological entropy $h=h(f)$ is 


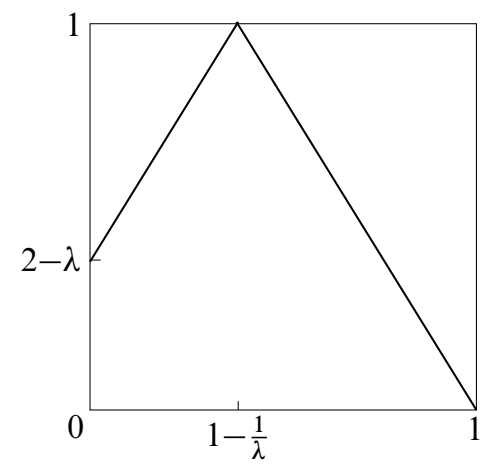

Figure 10: Graph of the tent map $T_{\lambda}$

semi-conjugate to the tent map of slope $e^{h}$ (see Milnor-Thurston [13]). In this section, only tent maps with slopes $\lambda \in(\sqrt{2}, 2]$ will be considered.

There is a countable dense subset $\Lambda \subset(\sqrt{2}, 2]$ of parameters $\lambda$ for which 1 is either a periodic $\left(T_{\lambda}^{n}(1)=1\right.$ for some $\left.n \geq 1\right)$ or a preperiodic $\left(T_{\lambda}^{k}(1)\right.$ is periodic for some $\left.k \geq 0\right)$ point of $T_{\lambda}$. In [6] the authors constructed, for each $\lambda \in \Lambda$, a complexification $f_{\lambda}: S_{\lambda} \rightarrow$ $S_{\lambda}$ of $T_{\lambda}$ as a quasi-conformal automorphism of a complex sphere $S_{\lambda}$. Naturally, one would like to be able to define such complexifications $f_{\lambda}$ for all $\lambda \in(\sqrt{2}, 2]$ by taking limits through $\Lambda$, and this was one of the motivations for the work described in the current paper. The above spheres $S_{\lambda}$ are constructed as paper spheres, and the first step in taking limits is to identify each $S_{\lambda}$ with the Riemann sphere $\widehat{\mathbb{C}}$ by a suitable choice of normalisation. In order to construct limits, it is necessary to show that the uniformising maps $u_{\lambda}: S_{\lambda} \rightarrow \widehat{\mathbb{C}}$ have a uniform modulus of continuity.

The family $\left(f_{\lambda}\right)_{\lambda \in \Lambda}$ contains a subfamily, parametrised by rationals $m / n \in(0,1 / 2)$, for which the paper sphere $S_{m / n}:=S_{\lambda}$ arises from a paper-folding scheme with only finitely many segment pairings: these are the so-called NBT examples of Hall [10]. In these cases, $f_{m / n}:=f_{\lambda}$ is a pseudo-Anosov automorphism: it preserves a transverse pair of measured foliations with finitely many singularities, stretching one foliation uniformly by a factor $\lambda$ and contracting the other uniformly by $1 / \lambda$ - the foliations are the projections to $S_{\lambda}$ of the horizontal and vertical foliations of the polygon $P_{\lambda}$. For all other $\lambda \in \Lambda, f_{\lambda}$ is a generalised pseudo-Anosov: these are defined in the same way as pseudo-Anosovs, except that their invariant foliations are permitted to have infinitely many singularities, provided that these singularities accumulate in only finitely many points.

The simplest example of a generalised pseudo-Anosov is the tight horseshoe, which is the complexification of the full tent map $T_{2}$. Its sphere $S=S_{2}$ of definition is obtained from the plain paper-folding scheme $(\Sigma, \mathcal{P})$, where $\Sigma=[0,1] \times[0,1] \subset \mathbb{R}^{2}$ 
and the segment pairings $\mathcal{P}$ consist of two folds of length $1 / 2^{i}$ for each $i \geq 1$ : the top and right sides of $\Sigma$ are folded in half, and the bottom and left sides are covered by folds of lengths $1 / 2^{i}$ for $i \geq 2$, arranged in order of decreasing length from the bottom right and top left vertices respectively (Figure 11). As in Example 63, the conditions of Theorem 59 hold at the unique singular point arising from the identification of the bottom left corner of $\Sigma$ with all of the fold endpoints, so that $S$ has a unique complex structure induced by the Euclidean structure on $\Sigma$. The scar $G$ is an $\infty$-od, having two edges of length $1 / 2^{i}$ for each $i \geq 1$.

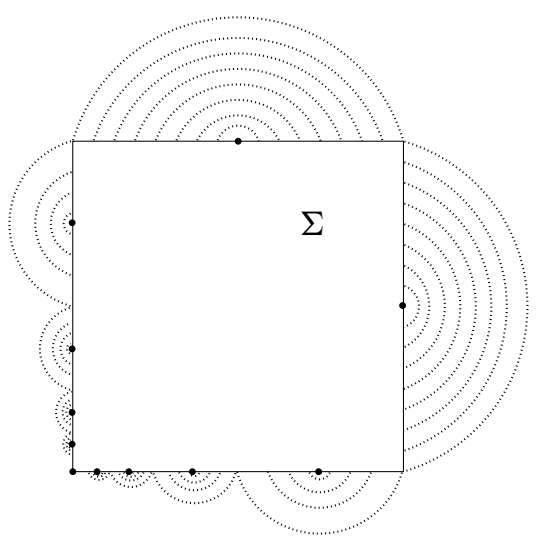

Figure 11: The sphere of definition of the tight horseshoe

Let $F: \Sigma \rightarrow \Sigma$ be the (discontinuous and non-injective) function defined by

$$
F(x, y)= \begin{cases}\left(2 x, \frac{y}{2}\right) & \text { if } x \leq \frac{1}{2} \\ \left(2(1-x), 1-\frac{y}{2}\right) & \text { if } x>\frac{1}{2}\end{cases}
$$

so that the first coordinate of $F(x, y)$ is $T_{2}(x)$, and $F$ contracts by a factor 2 in the vertical direction. The identifications on $\Sigma$ are precisely those which are needed to make $F$ continuous and injective, and it therefore descends to a homeomorphism $f: S \rightarrow S$. This homeomorphism preserves the foliations on $S$ which are the projections of the horizontal and vertical foliations of $\Sigma$, stretching the former by a factor 2 and contracting the latter by a factor $1 / 2$ : the foliations have 1 -pronged singularities corresponding to the mid-point of each fold, which accumulate on the unique singular point. $f$ is called the tight horseshoe because it can be obtained from Smale's horseshoe map [18] by collapsing the gaps in the non-wandering Cantor set.

The example elaborated in this section treats the convergence of the NBT pseudoAnosov homeomorphisms $f_{1 / n}: S_{1 / n} \rightarrow S_{1 / n}$ (which will henceforth be denoted $f_{n}: S_{n} \rightarrow S_{n}$ ) to the tight horseshoe $f: S \rightarrow S$ as $n \rightarrow \infty$ : uniformising maps 
$u_{n}: S_{n} \rightarrow \widehat{\mathbb{C}}$ and $u: S \rightarrow \widehat{\mathbb{C}}$ are chosen so that the homeomorphisms $u_{n} \circ f_{n} \circ u_{n}^{-1}$ of $\widehat{\mathbb{C}}$ converge to $u \circ f \circ u^{-1}$. This will provide a dynamical meaning to the term tight horseshoe. It was shown in [10] that the sequence $\left(f_{n}\right)$ effectively exhausts the finite invariant sets of the horseshoe, in the sense that for any finite invariant set $A$ of the horseshoe, there is some $N$ such that, for all $n \geq N, f_{n}$ is the pseudoAnosov representative in the isotopy class of the horseshoe relative to a finite invariant set $A^{\prime} \supset A$ : thus the tight horseshoe is tight in the sense of the introduction to this article.

The paper-folding schemes $\left(P_{n}, \mathcal{P}_{n}\right)$ which provide the spheres $S_{n}$, and the homeomorphisms $f_{n}: S_{n} \rightarrow S_{n}$ will be described in Section 7.2. This application of the results of the article has been chosen so that an elementary and self-contained description of these spaces and functions can be given without requiring the machinery of train tracks. However, the price for eschewing this machinery is that the constructions are both ad-hoc and involved. In Section 7.3 it is shown that there are uniform polygon constants for the polygons $P_{n}$, which makes it possible, in Section 7.4, to obtain a uniform modulus of continuity for the uniformising maps $\phi_{n}: P_{n} \rightarrow \widehat{\mathbb{C}}$ and hence to complete the proof of convergence.

\subsection{Paper spheres $S_{n}$ and pseudo-Anosov homeomorphisms $f_{n}: S_{n} \rightarrow S_{n}$}

Let $\lambda \in(\sqrt{2}, 2]$. The itinerary $k_{\lambda}(x) \in\{0, C, 1\}^{\mathbb{N}}$ of a point $x \in[0,1]$ under the tent map $T_{\lambda}$ is defined by

$$
k_{\lambda}(x)_{i}= \begin{cases}0 & \text { if } T_{\lambda}^{i}(x)<1-\frac{1}{\lambda}, \\ C & \text { if } T_{\lambda}^{i}(x)=1-\frac{1}{\lambda}, \\ 1 & \text { if } T_{\lambda}^{i}(x)>1-\frac{1}{\lambda} .\end{cases}
$$

The kneading invariant of $T_{\lambda}$ is defined as

$$
k\left(T_{\lambda}\right)=k_{\lambda}(1) .
$$

The kneading theory of Milnor and Thurston [13] provides a way of understanding the dynamics of $T_{\lambda}$ by means of its kneading invariant. In particular, $\lambda$ itself can be recovered as the reciprocal of the smallest positive root $\mu$ of a certain power series. In the case where 1 is a period $N$ point of $T_{\lambda}, \mu$ is the smallest positive root of the polynomial $\sum_{i=0}^{N-1} \theta_{i} t^{i}$ whose coefficients $\theta_{i} \in\{-1,+1\}$ are given by $\theta_{0}=1$ and

$$
\theta_{i}=\left\{\begin{aligned}
\theta_{i-1} & \text { if } k\left(T_{\lambda}\right)_{i-1}=0 \\
-\theta_{i-1} & \text { if } k\left(T_{\lambda}\right)_{i-1}=1
\end{aligned}\right.
$$

for $1 \leq i<N$. 
Let $n \geq 3$. The value $\lambda_{n}$ of $\lambda$ corresponding to the $1 / n$ NBT case (which are the ones of interest in this section) is the slope of the tent map with kneading invariant

$$
k\left(T_{\lambda}\right)=\left(10^{n-1} 1 C\right)^{\infty}
$$

( $n$ will be fixed throughout most this subsection, and hence the suffix $n$ on $\lambda_{n}$ will usually be omitted). Then $\mu$ is the smallest positive root of the polynomial

$$
f_{n}(t)=1-t-t^{2}-\cdots-t^{n}+t^{n+1},
$$

and $\lambda=1 / \mu$ is the largest root of the same polynomial. In particular, multiplying through by $(t-1), \lambda$ satisfies

$$
\lambda^{n+2}-2 \lambda^{n+1}+2 \lambda-1=0 .
$$

$\left(\lambda_{n}\right)$ is therefore an increasing sequence with $\lambda_{n} \rightarrow 2$ as $n \rightarrow \infty$.

Since $k\left(T_{\lambda}\right)=\left(10^{n-1} 1 C\right)^{\infty}, 1$ is a period $n+2$ point of $T_{\lambda}$ : write $p_{0}=1$ and $p_{i}=T_{\lambda}^{i}\left(p_{0}\right)$ for $1 \leq i \leq n+1$ so that $T_{\lambda}\left(p_{n+1}\right)=p_{0}$. The following explicit description of the points $p_{i}$ is easily verified by induction:

$$
p_{i}= \begin{cases}1 & \text { if } i=0, \\ 0 & \text { if } i=1, \\ \frac{(2-\lambda)\left(\lambda^{i-1}-1\right)}{\lambda-1} & \text { if } 2 \leq i \leq n, \\ 1-\frac{1}{\lambda} & \text { if } i=n+1 .\end{cases}
$$

Now define a function $F_{n}: \Sigma \rightarrow \mathbb{R}^{2}$ by

$$
F_{n}(x, y)= \begin{cases}\left(\lambda_{n}(x-1)+2, \frac{y}{\lambda_{n}}-\frac{1}{\lambda_{n}^{n+1}+1}\right) & \text { if } x \leq 1-\frac{1}{\lambda_{n}}, \\ \left(\lambda_{n}(1-x), 1-\frac{y}{\lambda_{n}}\right) & \text { if } x>1-\frac{1}{\lambda_{n}}\end{cases}
$$

so that the first coordinate of $F_{n}(x, y)$ is $T_{\lambda_{n}}(x)$, and $F_{n}$ contracts by a factor $1 / \lambda_{n}$ in the $y$ direction (see Figure 12). Notice that $F_{n}$ is not injective, and that it is discontinuous across the line $x=1-1 / \lambda_{n}$.

The polygon $P_{n}$ in the paper-folding scheme $\left(P_{n}, \mathcal{P}_{n}\right)$ is chosen so that $F_{n}\left(P_{n}\right)=P_{n}$ and $F_{n}$ is injective on its interior. The segment pairings $\mathcal{P}_{n}$ are then chosen to make the induced action of $F_{n}$ on the quotient paper sphere $S_{n}$ continuous and injective. The resulting homeomorphism $f_{n}: S_{n} \rightarrow S_{n}$ will be a pseudo-Anosov map, with $n+2$ one-pronged singularities, and one $n$-pronged singularity.

$P_{n}$ has $n+2$ horizontal sides and $n+2$ vertical sides. The vertical sides $V_{i}(0 \leq i \leq$ $n+1)$ are contained in the vertical fibers over the points $p_{i}$ of the orbit of 1 under the 


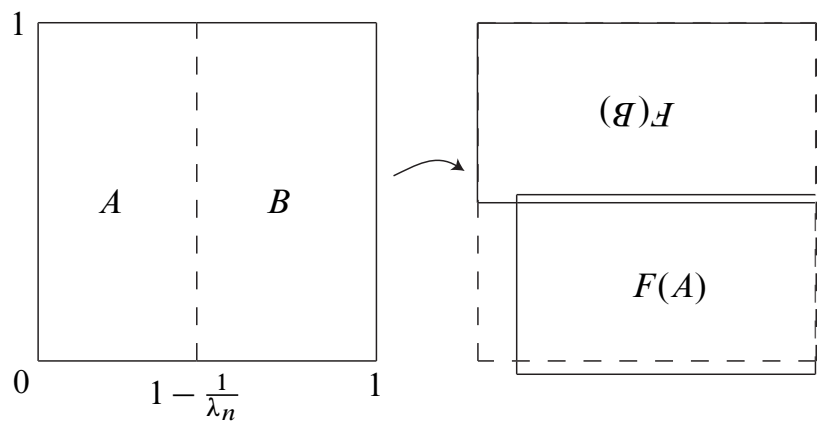

Figure 12: The action of $F_{n}: \Sigma \rightarrow \mathbb{R}^{2}$

tent map $T_{\lambda}$ : they are defined by

$$
V_{i}= \begin{cases}\{1\} \times\left[0, \frac{\lambda^{n+1}}{\lambda^{n+1}+1}\right] & \text { if } i=0 \\ F_{n}^{i}\left(V_{0}\right) & \text { if } 1 \leq i \leq n+1\end{cases}
$$

By (23), the height $h$ of $V_{0}$ satisfies

$$
h=\frac{\lambda^{n+1}}{\lambda^{n+1}+1}=\frac{2 \lambda^{n+1}(\lambda-1)}{\lambda^{n+2}-1}
$$

so that the sum $\sum_{i=0}^{n+1} h / \lambda^{i}$ of the heights of the vertical sides is equal to 2 .

The essential point about the configuration of these vertical sides is contained in the following lemma (see Figure 13).

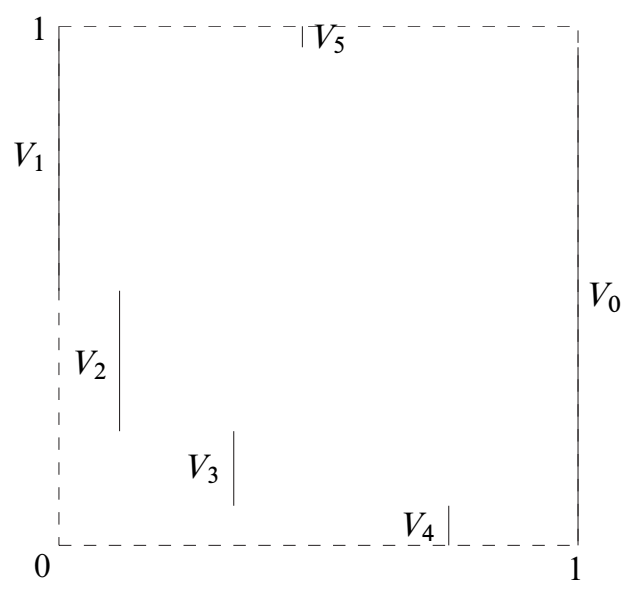

Figure 13: The vertical sides of $P_{4}$

Lemma 94 Let $\pi_{y}: \Sigma \rightarrow[0,1]$ be projection onto the second coordinate. Then 
(a) $\pi_{y}\left(V_{0} \cup V_{n+1}\right)=[0,1]$, and $\pi_{y}\left(V_{0}\right) \cap \pi_{y}\left(V_{n+1}\right)$ is a single point.

(b) $\pi_{y}\left(V_{1} \cup \ldots \cup V_{n}\right)=[0,1]$; and for $1 \leq i<j \leq n, \pi_{y}\left(V_{i}\right) \cap \pi_{y}\left(V_{j}\right)$ is a single point if $j=i+1$, and is empty otherwise.

Proof Recall that $V_{0}$ has height

$$
\left|V_{0}\right|=h=\frac{\lambda^{n+1}}{\lambda^{n+1}+1} .
$$

Since $F_{n}(1,0)=(0,1)$ and $\left|V_{1}\right|=h / \lambda$, it follows that

$$
V_{1}=\{0\} \times\left[1-\frac{h}{\lambda}, 1\right] .
$$

Now, using (23),

$$
F_{n}(0,1)=\left(p_{2}, \frac{1}{\lambda}-\frac{1}{\lambda^{n+1}+1}\right)=\left(p_{2}, 1-\frac{h}{\lambda}\right) .
$$

That is, the top point of $V_{2}$ is on the same horizontal level as the bottom point of $V_{1}$. Since $F_{n}$ preserves horizontal and vertical lines in $x \leq 1-1 / \lambda$, the top point of $V_{i+1}$ is on the same horizontal level as the bottom point of $V_{i}$ for $1 \leq i \leq n-1$. Since

$$
\sum_{i=1}^{n}\left|V_{i}\right|=h \sum_{i=1}^{n} \frac{1}{\lambda^{i}}=\frac{\lambda^{n+1}-\lambda}{\lambda^{n+2}-\lambda^{n+1}+\lambda-1}=1
$$

statement (b) of the lemma follows.

In particular,

$$
V_{n}=\left\{p_{n}\right\} \times\left[0, \frac{h}{\lambda^{n}}\right],
$$

and since $F_{n}\left(p_{n}, 0\right)=\left(p_{n+1}, 1\right)$ it follows that

$$
V_{n+1}=\left\{p_{n+1}\right\} \times\left[1-\frac{h}{\lambda^{n+1}}, 1\right] .
$$

Now $1-h / \lambda^{n+1}=\lambda^{n+1} /\left(\lambda^{n+1}+1\right)$, the vertical coordinate of the top of $V_{0}$, so statement (a) follows also.

Horizontal sides $H_{0}, \ldots, H_{n+1}$ can therefore be added to join the endpoints of the vertical sides and bound the polygon $P_{n}$. Specifically, let

- $H_{0}$ join the top of $V_{0}$ to the bottom of $V_{n+1}$;

- $H_{i}$ join the bottom of $V_{i}$ to the top of $V_{i+1}$ for $1 \leq i \leq n-1$;

- $H_{n}$ join the bottom of $V_{n}$ to the bottom of $V_{0}$, and 
- $H_{n+1}$ join the top of $V_{n+1}$ to the top of $V_{1}$

(see Figure 14). In particular, $H_{n-1}$ is the only horizontal side which intersects the line of discontinuity $x=p_{n+1}=1-1 / \lambda$ in its interior.

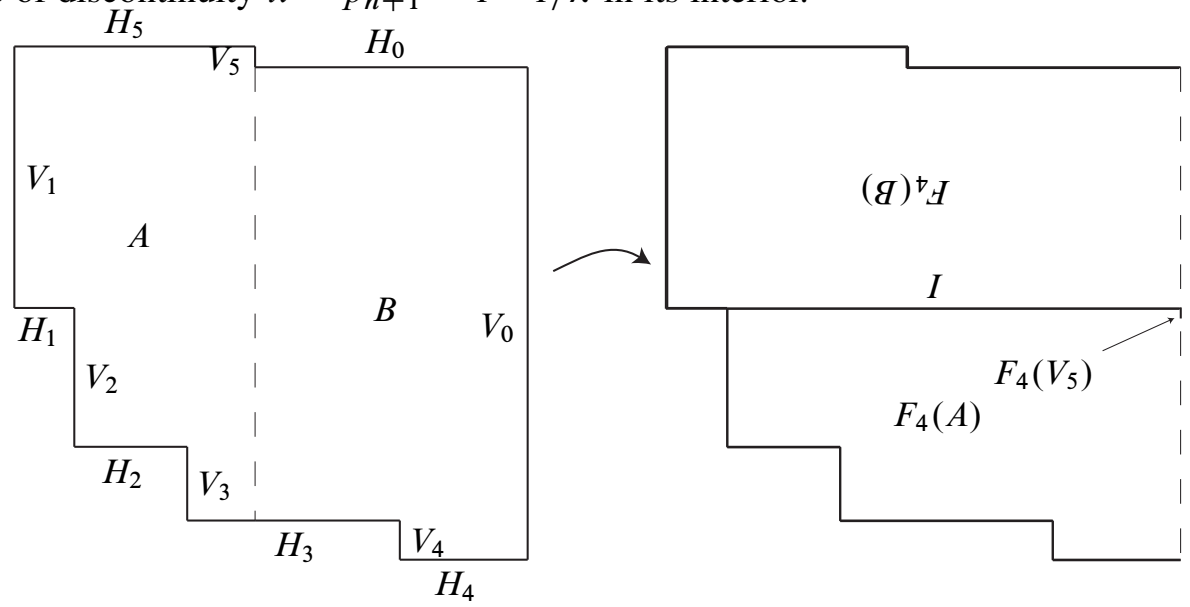

Figure 14: The polygon $P_{4}$ and its image under $F_{4}$

Next, the segment pairings on $\partial P_{n}$ which make $F_{n}$ continuous and injective will be described. The pairings on the vertical sides are straightforward and are treated first.

Lemma 95 The image under $F_{n}$ of the midpoint of $V_{n+1}$ is the midpoint of $V_{0}$.

Proof The midpoint of $V_{n+1}$ has vertical coordinate $1-h / 2 \lambda^{n+1}$, and so its image has vertical coordinate

$$
\frac{1-h / 2 \lambda^{n+1}}{\lambda}-\frac{1}{\lambda^{n+1}+1}=\frac{1}{\lambda}-\frac{1}{2 \lambda\left(\lambda^{n+1}+1\right)}-\frac{1}{\lambda^{n+1}+1} .
$$

Using (23), this is equal to $\frac{\lambda^{n+1}}{2\left(\lambda^{n+1}+1\right)}$, the vertical coordinate of the midpoint of $V_{0}$.

Define segment pairings on the vertical sides of $P_{n}$ by folding each side $V_{i}$ about its midpoint. Since $V_{i}$ is mapped affinely onto $V_{i+1}$ for $0 \leq i \leq n$, and $V_{n+1}$ is mapped affinely into $V_{0}$ with the midpoint being sent to the midpoint, the identifications arising from these pairings are respected by the action of $F_{n}$. Moreover, the identifications make $F_{n}$ continuous across the line $x=1-1 / \lambda$.

Write $\widetilde{P}_{n}$ for the quotient space under these identifications on the vertical sides (a disk with boundary $\bigcup H_{i}$ ), so that $F_{n}$ induces a continuous surjection, $\widetilde{F}_{n}: \widetilde{P}_{n} \rightarrow \widetilde{P}_{n}$.

The purpose of the segment pairings on the horizontal sides of $P_{n}$ is to make $\widetilde{F}_{n}$ injective: they are more complicated to describe. Observe first how $F_{n}$ acts on these sides: 
- $F_{n}\left(H_{0}\right)=I \cup H_{1}$, where $I$ is the horizontal segment joining the right hand end of $H_{1}$ to $V_{0}$ (see Figure 14). Write $H_{0}=H_{0}^{\prime} \cup H_{0}^{\prime \prime}$, where $F_{n}\left(H_{0}^{\prime}\right)=I$ and $F_{n}\left(H_{0}^{\prime \prime}\right)=H_{1}$;

- $F_{n}\left(H_{i}\right)=H_{i+1}$ for $1 \leq i \leq n-2$;

- $F_{n}\left(H_{n-1}\right)=H_{n} \cup H_{0}$ (recall that $H_{n-1}$ crosses the line of discontinuity $x=$ $1-1 / \lambda)$

- $\quad F_{n}\left(H_{n}\right)=H_{n+1}$; and

- $F_{n}\left(H_{n+1}\right)=I$.

In order to make $F_{n}$ injective, it is therefore necessary to identify $H_{n+1}$ with $H_{0}^{\prime}$, and then to propagate this identification under the dynamics.

Writing $I \rightarrow J$ to mean $F_{n}(I) \supset J$, observe that

$$
H_{0}^{\prime \prime} \rightarrow H_{1} \rightarrow H_{2} \rightarrow \cdots \rightarrow H_{n-1} \rightarrow H_{0}^{\prime \prime} .
$$

There is therefore a period $n$ point $q_{0}$ of $F_{n}$ in $H_{0}^{\prime \prime}$, the points $q_{i}=F_{n}^{i}\left(q_{0}\right)(1 \leq i<n)$ of whose orbit satisfy $q_{i} \in H_{i}$, and are therefore ordered cyclically according to their indices around the boundary of $\widetilde{P}_{n}$.

Lemma 96 The horizontal coordinate of $q_{0}$ is $1-\frac{2-\lambda}{\lambda(\lambda+1)}$. Moreover, the distance from the left hand endpoint of $H_{0}$ to $q_{0}$ is equal to the length of $H_{n+1}$ plus the distance from the left hand endpoint of $H_{1}$ to $q_{1}$.

Proof Let $L$ be the segment of $H_{0}$ from its left hand end to $q_{0}$, and $M$ be the segment of $H_{1}$ from $q_{1}$ to its right hand end (see Figure 15). Then $F_{n}(L)=I \cup M$ and $F_{n}^{n-1}(M)=L$. Thus

$$
\lambda|L|=|I|+|M|=\lambda-1+|M|
$$

(using that the right hand endpoint of $H_{1}$ has horizontal coordinate $2-\lambda$ ), and

$$
\lambda^{n-1}|M|=|L| \text {. }
$$

These give $|L|=\lambda^{n-1}(\lambda-1) /\left(\lambda^{n}-1\right)$, and hence the horizontal coordinate $\xi$ of $q_{0}$, given by $\xi=1-\frac{1}{\lambda}+|L|$, is

$$
\xi=1-\frac{1}{\lambda}+\frac{\lambda^{n-1}(\lambda-1)}{\lambda^{n}-1}
$$

which simplifies to the required expression using (23). 


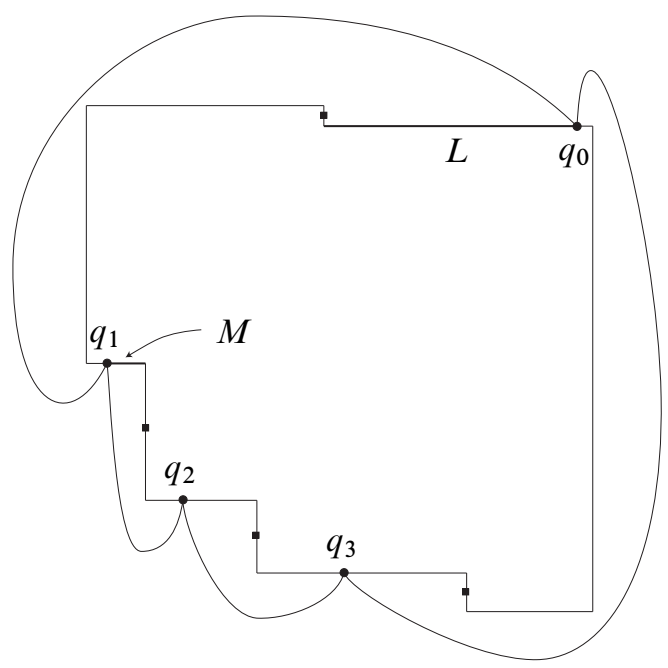

Figure 15: Identifications on the horizontal sides of $P_{4}$.

Now $\left|H_{n+1}\right|=1-1 / \lambda$, and the distance from the left hand endpoint of $H_{1}$ to $q_{1}$ is

$$
\left|H_{1}\right|-|M|=2-\lambda-\frac{|L|}{\lambda^{n-1}}=2-\lambda-\frac{\lambda-1}{\lambda^{n}-1},
$$

and the sum of these simplifies, using (23), to $|L|$ as required.

It follows that identifying $L$ with the union of $H_{n+1}$ and the segment of $H_{1}$ between its left hand endpoint and $q_{1}$ identifies $H_{n+1}$ with $H_{0}^{\prime}$ as required. Propagating this identification under the dynamics requires identifying the two halves of the segment of the boundary of $\widetilde{P}_{n}$ between $q_{i}$ and $q_{i+1}$ for each $0 \leq i<n-1$, and the two halves of the segment between $q_{n-1}$ and $q_{0}$ (Figure 15). Each of these identifications can be realised as a segment pairing on $P_{n}$, with the exception of the identification of $L$ with $H_{n+1}$ and a segment of $H_{1}$, and the identification of the two halves of the segment between $q_{n-1}$ and $q_{0}$, each of which can be expressed as two segment pairings. There is therefore a total of $n+2$ horizontal segment pairings, in addition to the $n+2$ vertical segment pairings.

The paper surface $S_{n}=P_{n} / d_{P_{n}}^{\mathcal{P}_{n}}$ is a sphere, on which the horizontal and vertical foliations of $P_{n}$ descend to a pair of transverse measured foliations, with $n+21$-pronged singularities (at the midpoints of the vertical sides) and an $n$-pronged singularity (at the points of the period $n$ orbit $Q$ on the horizontal boundary, which are all identified). Since $F_{n}$ stretches in the horizontal direction by a factor $\lambda$, and contracts in the vertical direction by a factor $1 / \lambda$, it induces a pseudo-Anosov homeomorphism $f_{n}: S_{n} \rightarrow S_{n}$ of the paper surface. 
The scar $G_{n}$ of $\left(P_{n}, \mathcal{P}_{n}\right)$ is a tree with $2 n+4$ edges corresponding to the $2 n+4$ segment pairings in $\mathcal{P}_{n}$ (Figure 16): however, $n$ of its vertices (the projections of the endpoints of $V_{i}$ for $2 \leq i \leq n+1$ ) have valence 2 . It will be convenient to describe it in the following way. Let $q_{0} \in G_{n}$ denote the projection of the periodic orbit $\left\{q_{0}, \ldots, q_{n-1}\right\}$ in $P_{n}: q_{0}$ will be called the central vertex of $G_{n}$. There are $n$ horizontal edges $h_{0}, \ldots, h_{n-1}$ of $G_{n}$ which emanate from $q_{0}$ and have lengths $\left|h_{i}\right|=\alpha \lambda^{i}$, where $\alpha=(\lambda-1) /\left(\lambda^{n}-1\right)$ so that $\sum_{i=0}^{n-1} \alpha \lambda^{i}=1$. Similarly there are $n+2$ vertical edges $v_{0}, \ldots, v_{n+1}$ of $G_{n}$, which have lengths $\left|v_{i}\right|=\beta \lambda^{i}$, where $\beta=(\lambda-1) /\left(\lambda^{n+2}-1\right)$. For $0 \leq i<n$, the edge $v_{i}$ emanates from the end of $h_{n-1-i}$. On the other hand $v_{n}$ (corresponding to $V_{1}$ ) emanates from $h_{n-1}$ at distance $\alpha / \lambda$ from $q_{0}$ (this distance comes from Lemma 96); and $v_{n+1}$ (corresponding to $V_{0}$ ) emanates from $h_{n-2}$ at distance $\alpha / \lambda^{2}$ from $q_{0}$. In particular, $h_{n-2}$ and $h_{n-1}$ are strictly speaking unions of two edges of $G_{n}$.

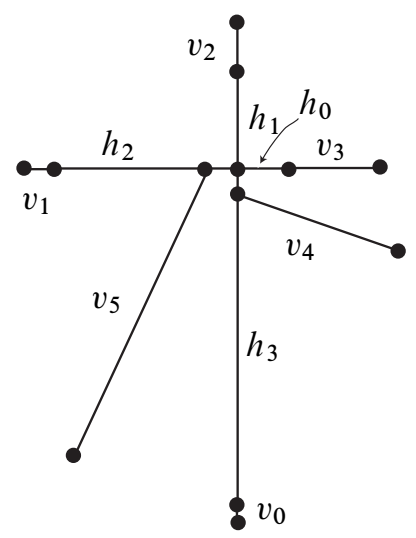

Figure 16: The scar of the $P_{4}$ paper surface

The final lemma of this section shows that the polygons $P_{n}$ converge to the square $\Sigma$ (see Figure 17), the equivalence relations $\sim_{\mathcal{P}_{n}}$ converge to the equivalence relation $\sim_{\mathcal{P}}$ of the tight horseshoe, and the functions $F_{n}: P_{n} \rightarrow P_{n}$ converge in an appropriate sense to $F: \Sigma \rightarrow \Sigma$. From this point on, superscripts $n$ will be added to indicate dependence on $n$ where they were previously omitted: thus, for example, the horizontal and vertical sides of $P_{n}$ will be denoted $H_{i}^{n}$ and $V_{i}^{n}$ for $0 \leq i \leq n+1$.

\section{Lemma 97}

(a) Let $\varepsilon>0$. Then the square $\Sigma_{\varepsilon}=[\varepsilon, 1-\varepsilon] \times[\varepsilon, 1-\varepsilon]$ is contained in $P_{n}$ for all sufficiently large $n$. In particular, the polygons $P_{n}$ converge in the Hausdorff metric to $\Sigma$. 

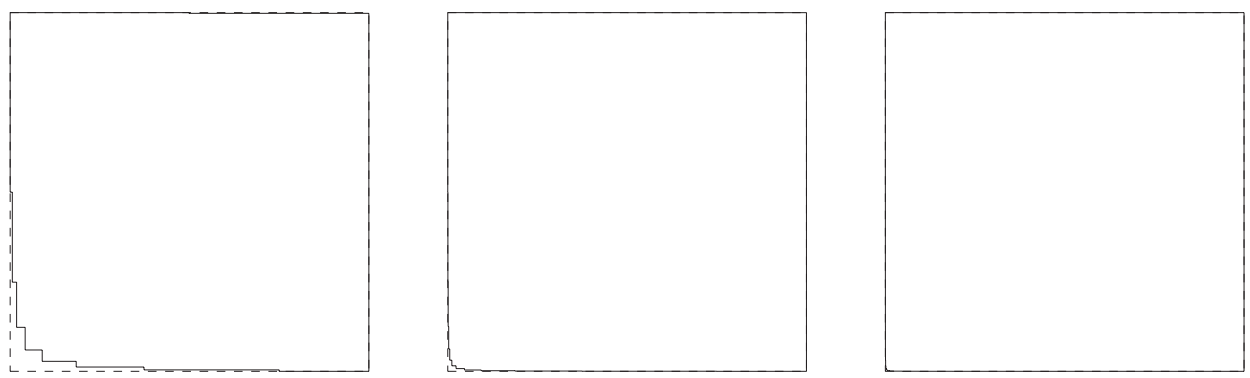

Figure 17: The polygons $P_{n}$ (here $n=8,12,16$ ) converge to $\Sigma$ (shown with dashed lines)

(b) The equivalence relations $\sim_{\mathcal{P}_{n}}$ converge in the Hausdorff metric to the equivalence relation $\sim_{\mathcal{P}}$.

(c) Let $\left(x_{n}, y_{n}\right) \rightarrow(x, y)$ be a convergent sequence in $\Sigma$ with $\left(x_{n}, y_{n}\right) \in P_{n}$ for all $n$. If $x \neq 1 / 2$ then $F_{n}\left(x_{n}, y_{n}\right) \rightarrow F(x, y)$. If $x=1 / 2$ then there is a subsequence $\left(x_{n_{i}}, y_{n_{i}}\right)$ such that $F_{n_{i}}\left(x_{n_{i}}, y_{n_{i}}\right) \rightarrow z \in \Sigma$ where $z \sim_{\mathcal{P}} F(x, y)$.

\section{Proof}

(a) It is enough to show that all of the sides of $P_{n}$ are contained in $C_{\varepsilon}=\Sigma \backslash \Sigma_{\varepsilon}$. $V_{0}^{n}, V_{1}^{n}, H_{n}^{n}$, and $H_{n+1}^{n}$, being subsets of $\partial \Sigma$, are contained in $C_{\varepsilon}$ for all $n$. Since $\lambda_{n}>3 / 2$ for all $n$ (as $\lambda_{3} \simeq 1.722$ ), the heights of the vertical sides satisfy $\left|V_{i}^{n}\right|<(2 / 3)^{i}$. Pick $K$ large enough that $\sum_{i=K}^{\infty}(2 / 3)^{i}<\varepsilon$ : then the vertical side $V_{i}^{n}$ is contained in $[0,1] \times([0, \varepsilon) \cup(1-\varepsilon, 1]) \subset C_{\varepsilon}$ whenever $i>K$ : this in turn means that $H_{i}^{n} \subset C_{\varepsilon}$ whenever $i \geq K$, and $H_{0}^{n} \subset C_{\varepsilon}$ provided that $n>K$. Now pick $N>K$ large enough that $2-\lambda_{N}<\varepsilon / 2^{K+1}$, and suppose that $n \geq N$. Then, by (24), whenever $2 \leq i \leq K$ the side $V_{i}^{n}$ has horizontal coordinate

$$
\begin{aligned}
p_{i}^{n} & =\frac{\left(2-\lambda_{n}\right)\left(\lambda_{n}^{i-1}-1\right)}{\lambda_{n}-1} \\
& <2\left(2-\lambda_{n}\right)\left(\lambda_{n}^{i-1}-1\right) \quad\left(\text { as } \lambda_{n}>3 / 2\right) \\
& <\varepsilon\left(\lambda_{n}^{i-1}-1\right) / 2^{K}<\varepsilon,
\end{aligned}
$$

and hence $V_{i}^{n} \subset[0, \varepsilon) \times[0,1] \subset C_{\varepsilon}$ : this in turn means that $H_{i-1}^{n} \subset C_{\varepsilon}$.

(b) Let $\varepsilon>0$. It is required to show that there is some $N$ such that, for all $n \geq N$ :

(i) If $a, b \in \Sigma$ with $a \sim_{\mathcal{P}} b$, then there exist $a^{\prime}, b^{\prime} \in P_{n}$ with $a^{\prime} \sim_{\mathcal{P}_{n}} b^{\prime}$ and $\left|a-a^{\prime}\right|<\varepsilon,\left|b-b^{\prime}\right|<\varepsilon$.

(ii) If $a, b \in P_{n}$ with $a \sim_{\mathcal{P}_{n}} b$, then there exist $a^{\prime}, b^{\prime} \in \Sigma$ with $a^{\prime} \sim_{\mathcal{P}} b^{\prime}$ and $\left|a-a^{\prime}\right|<\varepsilon,\left|b-b^{\prime}\right|<\varepsilon$. 
There are four cases to consider:

Case $1 a=b$.

This is dealt with by (a).

Case $2 a$ and $b$ are an interior pair of a vertical segment pairing.

Choose $K$ so that $\sum_{i=1}^{K}\left|V_{i}^{n}\right|>1-\varepsilon / 2$ for all $n>K$. For each $i$ with $0 \leq i \leq K$, $\left|V_{i}^{n}\right|=\lambda_{n}^{n+1-i} /\left(\lambda_{n}^{n+1}+1\right)$ converges to $1 / 2^{i}$ as $n \rightarrow \infty$. Thus, for sufficiently large $n$ :

- $V_{0}^{n}$ is within Hausdorff distance $\varepsilon / 2$ of $\{1\} \times[0,1]$;

- $V_{i}^{n}$ is within Hausdorff distance $\varepsilon / 2$ of $\{0\} \times\left[1 / 2^{i}, 1 / 2^{i-1}\right]$ for $1 \leq i \leq K$; and

- $\left|V_{i}^{n}\right|<\varepsilon / 2$ for $K<i \leq n+1$.

That is, each folded vertical segment in $P_{n}$ (respectively $\Sigma$ ) is either very close to a folded vertical segment in $\Sigma$ (respectively $P_{n}$ ), or is very small. In the former case, $(a, b)$ can be approximated by some $\left(a^{\prime}, b^{\prime}\right)$; and in the latter case, it can be approximated by some $\left(a^{\prime}, a^{\prime}\right)$.

Case $3 a$ and $b$ are an interior pair of a horizontal segment pairing.

The argument is similar to that of the second case, except that (see Figure 15) the relevant quantities are the horizontal coordinates of the points $\left\{q_{0}^{n}, q_{1}^{n}, \ldots, q_{n-1}^{n}\right\}$ of the periodic orbit of $F_{n}$ on $\partial P_{n}$. When $n$ is large, the horizontal coordinate of $q_{0}^{n}$ is very close to 1 , and the horizontal coordinates of $q_{i}^{n}$ for $1 \leq i<n$ are either very small, or very close to $1 / 2^{n-i}$.

Case $4 a$ and $b$ are endpoints of segment pairings.

The argument is an extension of the third case. When $n$ is large, the set of segment endpoints on $\partial \Sigma$ is Hausdorff close to the union of the periodic orbit of $F_{n}$ on $\partial P_{n}$ with the points $(1,0),(0,1)$ of $\partial P_{n}$ : these two points are very close to being identified with $q_{0}^{n}$ and $q_{1}^{n}$ respectively.

(c) If $x<1 / 2\left(\right.$ respectively $x>1 / 2$ ) then $x_{n}<1-1 / \lambda_{n}$ (respectively $x_{n}>1-1 / \lambda_{n}$ ) for all sufficiently large $n$, and the result is immediate from the definitions of $F_{n}$ and $F$.

If $x=1 / 2$ then $F(x, y)=(1, y / 2)$. Take a subsequence $\left(x_{n_{i}}, y_{n_{i}}\right)$ such that either $x_{n_{i}}<1-1 / \lambda_{n_{i}}$ for all $i$, or $x_{n_{i}}>1-1 / \lambda_{n_{i}}$ for all $i$. Then $F_{n_{i}}\left(x_{n_{i}}, y_{n_{i}}\right)$ converges to $(1, y / 2)$ in the former case, and to $(1,1-y / 2) \sim_{\mathcal{P}}(1, y / 2)$ in the latter case.

\subsection{Polygon constants}

Recall (Definition 68) that the polygon constants of a polygon $P$ are: the length $|\partial P|$ of its boundary; the collaring height $\bar{h}>0$ (Section 5.3.1); and $\bar{r}>0$, which in the 
plain case can be chosen arbitrarily. In this section it is shown that these constants can be chosen uniformly for the polygons $P_{n}$. Since $\left|\partial P_{n}\right|=4$ for all $n$, and $\bar{r}$ is arbitrary, the only issue is to find a uniform collaring height $\bar{h}$. It will be shown that $\bar{h}=\frac{1}{24}$ is a collaring height for all $n$, and the constant $\bar{r}$ will then be chosen as

$$
\bar{r}=\bar{h}=\frac{1}{24} .
$$

Observe (Figure 18) that the trapezoid of height $\bar{h}=1 / 24$ on every side of $P_{n}$ except for $V_{0}^{n}, V_{1}^{n}, H_{n}^{n}$, and $H_{n+1}^{n}$ is a parallelogram. However, the lengths of these four exceptional sides are uniformly bounded below:

$$
\begin{aligned}
& \left|V_{0}^{n}\right|=\frac{\lambda_{n}^{n+1}}{\lambda_{n}^{n+1}+1}>\frac{1}{2}, \\
& \left|V_{1}^{n}\right|=\frac{\left|V_{0}^{n}\right|}{\lambda_{n}}>\frac{1}{4}, \\
& \left|H_{n+1}^{n}\right|=1-\frac{1}{\lambda_{n}}>\frac{1}{3} \\
& \text { and } \quad\left|H_{n}^{n}\right|=\frac{\left|H_{n+1}^{n}\right|}{\lambda_{n}}>\frac{1}{6} \text {. }
\end{aligned}
$$

Hence the ratio between the lengths of the bases and the tops of the trapezoids on these sides lies in $[1 / 2,2]$ since $\bar{h} \leq \frac{1}{6} \times \frac{1}{4}=\frac{1}{24}$. (Since the base angles are $\pi / 4$, the difference between the length of the base and the length of the top is $2 \bar{h}$.) It therefore only remains to show that the trapezoids of height $\bar{h}$ only intersect along their common vertical sides. This is an immediate consequence of the observation that the difference between the vertical coordinates of the top of $V_{0}^{n}$ and the bottom of $V_{1}^{n}$ is

$$
\frac{\lambda_{n}^{n+1}}{\lambda_{n}^{n+1}+1}-\left(1-\frac{\lambda_{n}^{n}}{\lambda_{n}^{n+1}+1}\right)=\frac{1-1 / \lambda_{n}^{n}}{\lambda_{n}+1 / \lambda_{n}^{n}}>\frac{1-(2 / 3)^{3}}{2+1}=\frac{19}{81}>2 \bar{h} .
$$

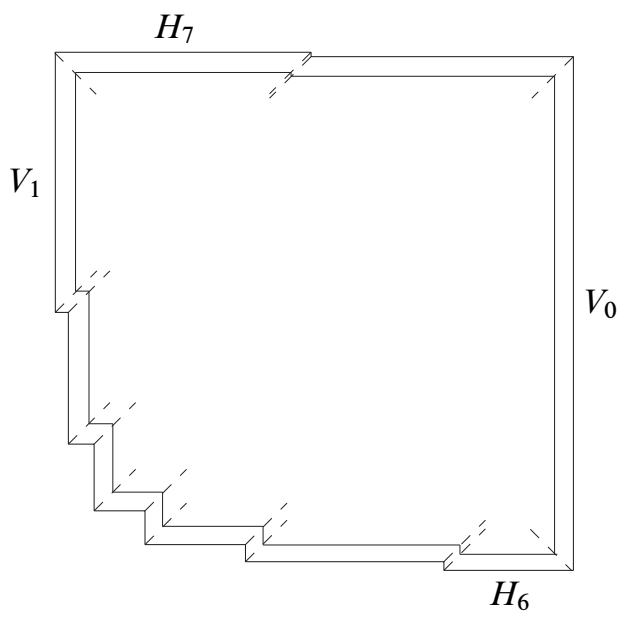

Figure 18: Collaring $P_{n}$ (here $n=6$ ) 


\subsection{Convergence of the uniformising maps}

Since the scars $G_{n}$ have no singular points, the paper spheres $S_{n}$ all have a natural complex structure, and there are unique uniformising maps $u_{n}: S_{n} \rightarrow \widehat{\mathbb{C}}$ such that the compositions $\phi_{n}=u_{n} \circ \pi_{n}: P_{n} \rightarrow \widehat{\mathbb{C}}$ satisfy $\phi_{n}(1,0)=0, \phi_{n}(1 / 2,1 / 2)=\infty$, and $\left(1 / \phi_{n}\right)^{\prime}(1 / 2,1 / 2)=1$ (the point $(1 / 2,1 / 2)$ lies in the complement of the height $\bar{h}$ collaring of $P_{n}$ for sufficiently large $n$ by Lemma 97(a): in fact it can be shown that this is true for all $n \geq 3$ ). The pseudo-Anosov homeomorphisms $f_{n}: S_{n} \rightarrow S_{n}$ induce homeomorphisms $\hat{f}_{n}: \widehat{\mathbb{C}} \rightarrow \widehat{\mathbb{C}}$ by $\hat{f}_{n}=u_{n} \circ f_{n} \circ u_{n}^{-1}$.

Similarly, recalling that $(\Sigma, \mathcal{P})$ is the paper-folding scheme used in the definition of the tight horseshoe $f: S \rightarrow S$, there is a unique uniformising map $u: S \rightarrow \widehat{\mathbb{C}}$ with the property that the composition $\phi=u \circ \pi: \Sigma \rightarrow \widehat{\mathbb{C}}$ satisfies $\phi(1,0)=0, \phi(1 / 2,1 / 2)=$ $\infty$, and $(1 / \phi)^{\prime}(1 / 2,1 / 2)=1$. The generalised pseudo-Anosov $f: S \rightarrow S$ induces a homeomorphism $\hat{f}: \widehat{\mathbb{C}} \rightarrow \widehat{\mathbb{C}}$.

In this section it is shown that the functions $\phi_{n}$ converge to $\phi$, and hence that the homeomorphisms $\hat{f}_{n}$ converge to $\hat{f}$. The key result, Lemma 100 below, is that the $\phi_{n}$ have a uniform modulus of continuity.

Definition $98(\mathcal{I}(t))$ Let $\mathcal{I}:(0, \bar{r}) \rightarrow(0, \infty)$ be the function defined by

$$
\mathcal{I}(t):=\frac{\ln 2}{12} \int_{t}^{\bar{r}} \frac{\mathrm{d} s}{s \ln \left(\frac{8}{s-t}\right)} .
$$

Remark $99 \mathcal{I}$ is a decreasing function, having derivative

$$
\mathcal{I}^{\prime}(t)=\frac{-\ln 2}{12} \int_{t}^{\bar{r}} \frac{\mathrm{d} s}{s(s-t)(\ln (8 /(s-t)))^{2}}<0 .
$$

Moreover $\mathcal{I}(t) \rightarrow \infty$ as $t \rightarrow 0$.

Lemma 100 Define $\bar{\rho}:[0, \delta) \rightarrow[0, \infty)$ by

$$
\bar{\rho}(t):= \begin{cases}0, & \text { if } t=0 ; \\ \max \left(\frac{16 R}{\exp (2 \pi M \mathcal{I}(2 A t))}, \kappa t\right), & \text { if } t>0 .\end{cases}
$$

Then for all $n \geq 3, \bar{\rho}$ is a modulus of continuity for $\phi_{n}: P_{n} \rightarrow \widehat{\mathbb{C}}$, with respect to the Euclidean metric on $P_{n}$ and the spherical metric on $\widehat{\mathbb{C}}$.

Here $\delta=1 / 4608$ and $A=96$ are given by (20), $M=1 / 5$ is given by Definition 77, $R=R(\bar{h})$ is given by Lemma 83 , and $\kappa=\kappa\left(\left|\partial P_{n}\right|, \delta\right)$ is given by (22). Observe that 
$\bar{\rho}$ is a modulus of continuity in the sense of Definition 79: it is continuous, positive, and strictly increasing in $(0, \delta)$ as the maximum of two functions with these properties; and $\bar{\rho}(t) \rightarrow 0$ as $t \rightarrow 0$ by Remark 99 .

Two preliminary lemmas are required.

Lemma 101 Let $n \geq 3$ and $r \in(0, \bar{r})$. Then, in $G_{n}$,

$$
m\left(q_{0} ; r\right) \leq 8 r \log _{2}(8 / r) \quad \text { and } \quad n\left(q_{0} ; r\right) \leq 4 \log _{2}(4 / r),
$$

where $q_{0}$ is the central vertex of $G_{n}$.

Proof The edges $v_{0}, v_{1}, v_{n}, v_{n+1}, h_{n-1}$ and $h_{n-2}$ of $G_{n}$ contribute at most $8 r$ to $m\left(q_{0} ; r\right)$ and at most 4 to $n\left(q_{0} ; r\right)$. The remaining edges form an $(n-2)-$ od, with edges of lengths

$$
\ell_{i}=\left|h_{i}\right|+\left|v_{n-1-i}\right|=\alpha \lambda^{i}+\beta \lambda^{n-1-i} \quad(0 \leq i<n-2) .
$$

Here $\alpha=\frac{1}{1+\lambda+\cdots+\lambda^{n-1}}<\frac{1}{\lambda^{n-1}}$ and $\beta=\frac{1}{1+\lambda+\cdots+\lambda^{n+1}}<\frac{1}{\lambda^{n+1}}$, so that

$$
\ell_{i}<\frac{1}{\lambda^{n-1-i}}+\frac{1}{\lambda^{i+2}}<\frac{1}{(\sqrt{2})^{n-1-i}}+\frac{1}{(\sqrt{2})^{i+2}}:=L_{i} .
$$

Let $k=2+\left\lceil 2 \log _{2}(1 / r)\right\rceil \leq 3+2 \log _{2}(1 / r)$ (where $\lceil x\rceil$ denotes the smallest integer which is not less than $x$ ), so that $\frac{1}{(\sqrt{2})^{k}} \leq \frac{r}{2}$. Then provided $n-1-i \geq k$ and $i+2 \geq k$ (that is, $k-2 \leq i \leq n-1-k$ ), $L_{i}$ satisfies

$$
L_{i}=\frac{1}{(\sqrt{2})^{n-1-i}}+\frac{1}{(\sqrt{2})^{i+2}} \leq \frac{2}{(\sqrt{2})^{k}} \leq r .
$$

There are at most $2 k-4$ values of $i$ which don't satisfy these inequalities, so that

$$
n\left(q_{0} ; r\right) \leq 4+(2 k-4) \leq 6+4 \log _{2}(1 / r)<4\left(2+\log _{2}(1 / r)\right)=4 \log _{2}(4 / r)
$$

as required. Similarly

$$
\begin{aligned}
m\left(q_{0} ; r\right) & \leq 8 r+(4 k-8) r+2 \sum_{i=k-2}^{n-1-k} L_{i} \\
& <8 r+(4 k-8) r+8 r \\
& <8 r\left(3+\log _{2}(1 / r)\right) \\
& =8 r \log _{2}(8 / r),
\end{aligned}
$$

since

$$
\sum_{i=k-2}^{n-1-k} L_{i}<\frac{2}{(\sqrt{2})^{k}} \sum_{i=0}^{\infty} \frac{1}{(\sqrt{2})^{i}}<\frac{8}{(\sqrt{2})^{k}} \leq 4 r .
$$


This completes the proof.

Lemma 102 For all $n \geq 3, q \in G_{n}$, and $t \in(0, \bar{r})$

$$
\int_{t}^{\bar{r}} \frac{\mathrm{d} s}{m(q ; s)+s \cdot n(q ; s)} \geq \mathcal{I}(t) \text {. }
$$

Proof Write $D:=d_{G_{n}}\left(q_{0}, q\right)$, where $q_{0}$ is the central vertex of $G_{n}$. Then

$$
m(q ; r) \leq \begin{cases}6 r, & \text { if } r \leq D ; \\ 6 D+m\left(q_{0} ; r-D\right) \leq 6 D+8(r-D) \log _{2}\left(\frac{8}{r-D}\right) & \text { if } r>D,\end{cases}
$$

and

$$
n(q ; r) \leq \begin{cases}3, & \text { if } r \leq D ; \\ 2+n\left(q_{0} ; r-D\right) \leq 2+4 \log _{2}\left(\frac{4}{r-D}\right)<4 \log _{2}\left(\frac{8}{r-D}\right) & \text { if } r>D .\end{cases}
$$

There are three cases to consider: $0 \leq D \leq t ; t \leq D \leq \bar{r}$; and $D \geq \bar{r}$.

Case $10 \leq D \leq t$.

Then

$$
\int_{t}^{\bar{r}} \frac{\mathrm{d} s}{m(q ; s)+s \cdot n(q ; s)} \geq \int_{t}^{\bar{r}} \frac{\mathrm{d} s}{6 D+(12 s-8 D) \log _{2}\left(\frac{8}{s-D}\right)} .
$$

Since $\log _{2}(8 /(s-D)) \geq \log _{2}(8 / \bar{r})>1$, this integral is in turn bounded below by

$$
F(D, t):=\int_{t}^{\bar{r}} \frac{\mathrm{d} s}{12 s \log _{2}\left(\frac{8}{s-D}\right)}=\frac{\ln 2}{12} \int_{t}^{\bar{r}} \frac{\mathrm{d} s}{s \ln \left(\frac{8}{s-D}\right)} .
$$

Now

$$
\frac{\partial F}{\partial D}=\frac{-\ln 2}{12} \int_{t}^{\bar{r}} \frac{\mathrm{d} s}{s(s-D)\left(\ln \left(\frac{8}{s-D}\right)\right)^{2}}<0
$$

for $D \in[0, t]$, so

$$
\int_{t}^{\bar{r}} \frac{\mathrm{d} s}{m(q ; s)+s \cdot n(q ; s)} \geq F(t, t)=\mathcal{I}(t)
$$

for all $q \in G_{n}$ with $d_{G_{n}}\left(q, q_{0}\right) \leq t$ as required.

Case $2 t \leq D \leq \bar{r}$.

Then

$\int_{t}^{\bar{r}} \frac{\mathrm{d} s}{m(q ; s)+s \cdot n(q ; s)} \geq \int_{t}^{D} \frac{\mathrm{d} s}{9 s}+F(D, D)=\frac{1}{9} \ln \left(\frac{D}{t}\right)+\frac{\ln 2}{12} \int_{D}^{\bar{r}} \frac{\mathrm{d} s}{s \ln \left(\frac{8}{s-D}\right)}$. 
The derivative of this lower bound with respect to $D$ is

$$
\begin{aligned}
\frac{1}{9 D}-\frac{\ln 2}{12} \int_{D}^{\bar{r}} \frac{\mathrm{d} s}{s(s-D)\left(\ln \left(\frac{8}{s-D}\right)\right)^{2}} & \geq \frac{1}{D}\left(\frac{1}{9}-\frac{\ln 2}{12} \int_{D}^{\bar{r}} \frac{\mathrm{d} s}{(s-D)\left(\ln \left(\frac{8}{s-D}\right)\right)^{2}}\right) \\
& =\frac{1}{D}\left(\frac{1}{9}-\frac{\ln 2}{12 \ln \left(\frac{8}{\bar{r}-D}\right)}\right) \\
& >\frac{1}{D}\left(\frac{1}{9}-\frac{\ln 2}{12 \ln (192)}\right)>0
\end{aligned}
$$

for $D \in[t, \bar{r}]$, using $\bar{r}=1 / 24$. Hence

$$
\int_{t}^{\bar{r}} \frac{\mathrm{d} s}{m(q ; s)+s \cdot n(q ; s)} \geq \frac{1}{9} \ln (1)+F(t, t)=\mathcal{I}(t)
$$

for all $q \in G_{n}$ with $t \leq d_{G_{n}}\left(q, q_{0}\right) \leq \bar{r}$ as required.

Case $3 \quad D \geq \bar{r}$.

Then

$$
\int_{t}^{\bar{r}} \frac{\mathrm{d} s}{m(q ; s)+s \cdot n(q ; s)} \geq \frac{1}{9} \int_{t}^{\bar{r}} \frac{\mathrm{d} s}{s}>\frac{\ln 2}{12} \int_{t}^{\bar{r}} \frac{\mathrm{d} s}{s \ln \left(\frac{8}{s-t}\right)}=\mathcal{I}(t)
$$

as required, using $\ln (8 /(s-t))>\ln (8 / \bar{r})>1$.

Proof of Lemma 100 Following the proof of Theorem 84, it is only required to show that the function $\rho_{q}(t)$ of (21) satisfies $\rho_{q}(t) \leq \bar{\rho}(t) / 2$ for all $n$, all $q \in Q_{n}(\delta)$, and all $t \in(0, \delta)$. (As in that proof, the factor 2 arises from the translation between the Euclidean and spherical metrics.)

So let $q \in Q_{n}(\delta)$ and $t \in(0, \delta)$. If $h_{q} \leq t$ then

$$
\begin{aligned}
\rho_{q}(t) & =\frac{8 R}{\exp \left(2 \pi \int_{A\left(t+h_{q}\right)}^{\bar{r}} \iota(\psi(q) ; s) \mathrm{d} s\right)} \\
& \leq \frac{8 R}{\exp \left(2 \pi \int_{2 A t}^{\bar{r}} \iota(\psi(q) ; s) \mathrm{d} s\right)} \\
& \leq \frac{8 R}{\exp (2 \pi M \mathcal{I}(2 A t))}
\end{aligned}
$$

by Lemma 102 , using $\iota(q ; s)=\frac{M}{m(q ; s)+s \cdot n(q ; s)}$.

On the other hand, if $h_{q}>t$ then an analogous argument gives

$$
\rho_{q}(t) \leq \frac{8 R t}{h_{q} \exp \left(2 \pi M \mathcal{I}\left(2 A h_{q}\right)\right)} .
$$


However the function $x \mapsto x \exp (2 \pi M \mathcal{I}(2 A x))$ is increasing on $(t, \delta]$, so

$$
\rho_{q}(t) \leq \frac{8 R t}{t \exp (2 \pi M \mathcal{I}(2 A t))}
$$

as required.

Lemma 103 For each $N \geq 3$ the sequence of functions $\left(\left.\phi_{n}\right|_{X_{N}}: X_{N} \rightarrow \widehat{\mathbb{C}}\right)_{n \geq N}$ converges uniformly to $\left.\phi\right|_{X_{N}}: X_{N} \rightarrow \widehat{\mathbb{C}}$, where $X_{N}=\bigcap_{n \geq N} P_{n}$. In particular, if $x_{n} \rightarrow x$ is a convergent sequence in $\Sigma$ with $x_{n} \in P_{n}$ for all $n$, then $\phi_{n}\left(x_{n}\right) \rightarrow \phi(x)$.

Remark 104 The sequence of polygons $\left(P_{n}\right)$ is not increasing, since the lengths $\left|V_{1}^{n}\right|=\lambda_{n}^{n} /\left(\lambda_{n}^{n+1}+1\right)$ of the sides $V_{1}^{n}$, which are contained in $\partial \Sigma$, decrease with $n$. However the sequence $\left(X_{n}\right)$ is increasing and converges Hausdorff to $\Sigma$ by Lemma 97(a).

Proof Since the sequence $\left(\phi_{n}\right)$ is equicontinuous and the sequence of domains $\left(P_{n}\right)$ converges Hausdorff to $\Sigma$, a variant of the Arzelà-Ascoli theorem (which is proved identically to the standard version) shows that there is a subsequence $\left(\phi_{n_{i}}\right)$ and a continuous function $\phi_{\infty}: \Sigma \rightarrow \widehat{\mathbb{C}}$ such that, for each $N \geq 3$, the sequence $\left(\left.\phi_{n_{i}}\right|_{X_{N}}\right)_{n_{i} \geq N}$ converges uniformly to $\left.\phi_{\infty}\right|_{X_{N}}$. It will be shown that $\phi_{\infty}=\phi$ for any such subsequence, which will establish the result.

Now $\phi_{\infty}(1 / 2,1 / 2)=\infty$ and $\phi_{\infty}(1,0)=0$, since $\phi_{n}(1 / 2,1 / 2)=\infty$ and $\phi_{n}(1,0)=0$ for all $n$. Moreover, because the functions $\phi_{n}$ are univalent on $\operatorname{Int}\left(P_{n}\right)$ and any open subset of $\operatorname{Int}(\Sigma)$ whose boundary is disjoint from $\partial \Sigma$ is contained in $X_{n}$ for sufficiently large $n$, the function $\phi_{\infty}$ is univalent in $\operatorname{Int}(\Sigma)$, and satisfies $\left(1 / \phi_{\infty}\right)^{\prime}(1 / 2,1 / 2)=1$. Because $\phi_{\infty}$ restricted to $\operatorname{Int}(\Sigma)$ is open and injective, there do not exist points $w \in \partial \Sigma$ and $z \in \operatorname{Int}(\Sigma)$ with $\phi_{\infty}(w)=\phi_{\infty}(z)$.

By Lemma 97(b), if $w, z \in \partial \Sigma$ with $w \sim_{\mathcal{P}} z$, then $\phi_{\infty}(w)=\phi_{\infty}(z)((w, z)$ is arbitrarily closely approximated by points $\left(w_{n}, z_{n}\right) \in \sim_{\mathcal{P}_{n}}$, which therefore satisfy $\left.\phi_{n}\left(w_{n}\right)=\phi_{n}\left(z_{n}\right)\right)$. There is therefore a continuous function $u_{\infty}: S \rightarrow \widehat{\mathbb{C}}$ with $\phi_{\infty}=$ $u_{\infty} \circ \pi$, which restricts to a conformal homeomorphism

$$
\left.u_{\infty}\right|_{S \backslash G}: S \backslash G \rightarrow \widehat{\mathbb{C}} \backslash u_{\infty}(G) .
$$

Let $z \in \partial \Sigma$ be any point in the interior of a paired segment $\alpha$ of $\mathcal{P}$, let $\varepsilon>0$ be the distance from $z$ to the nearest endpoint of $\alpha$, and let $z^{\prime}$ be the point of the paired segment $\alpha^{\prime}$ which is paired with $z$. Let $\Sigma^{z} \subset \mathbb{C}$ be obtained from $\Sigma$ by excising $B_{\Sigma}\left(z^{\prime} ; \varepsilon / 2\right)$ and gluing this half-disk onto $\Sigma$ near $z$ according to the pairing $\left\langle\alpha, \alpha^{\prime}\right\rangle$. Then $\phi_{\infty}$ induces a natural function $\phi_{\infty}^{z}: \Sigma^{z} \rightarrow \widehat{\mathbb{C}}$.

Choose sequences $z_{n} \rightarrow z$ and $z_{n}^{\prime} \rightarrow z^{\prime}$ where $z_{n}, z_{n}^{\prime} \in P_{n}$ and $z_{n} \sim \mathcal{P}_{n} z_{n}^{\prime}$. Then for sufficiently large $n$ an analogous construction can be carried out to obtain functions 
$\phi_{n}^{z}: P_{n}^{z} \rightarrow P_{n}^{z}$ which are univalent on $\operatorname{Int}\left(P_{n}^{z}\right)$. It follows that $\phi_{\infty}^{z}$ is univalent on $\operatorname{Int}\left(\Sigma^{z}\right)$, and in particular that there is no point $w \in \partial \Sigma$ with $\phi_{\infty}(w)=\phi_{\infty}(z)$ other than $z^{\prime}$.

Now if $w, z \in G$ with $w \neq z$ and $u_{\infty}(w)=u_{\infty}(z)$, then every point except possibly one of the interval $[w, z]_{G}$ is identified with another such point by $u_{\infty}$, as otherwise $u_{\infty}(G)$ would contain a simple closed curve, contradicting the fact that its complement is connected; in particular, $u_{\infty}$ identifies some point in the interior of a paired segment with another point which it is not $\sim_{\mathcal{P}}$-equivalent to. Since this has been shown to be impossible, $u_{\infty}$ is injective on $G$.

It follows that $u_{\infty}: S \rightarrow \widehat{\mathbb{C}}$ is a homeomorphism: since it is conformal on $S \backslash G$, and $G$ has finite 1-dimensional Hausdorff measure by Lemma 33(c), $u_{\infty}$ is conformal by Theorem 27. Because $u_{\infty}$ is normalised in the same way as $u$ the two are equal, and hence $\phi_{\infty}=u_{\infty} \circ \pi=u \circ \pi=\phi$ as required.

Theorem 105 The pseudo-Anosov homeomorphisms $\widehat{f_{n}}: \widehat{\mathbb{C}} \rightarrow \widehat{\mathbb{C}}$ converge pointwise to the generalised pseudo-Anosov $\hat{f}: \widehat{\mathbb{C}} \rightarrow \widehat{\mathbb{C}}$.

Proof Let $z \in \widehat{\mathbb{C}}$ and choose, for each $n$, a point $x_{n} \in P_{n}$ with $\phi_{n}\left(x_{n}\right)=z$ : thus $\hat{f}_{n}(z)=\phi_{n}\left(F_{n}\left(x_{n}\right)\right)$. It will be shown that if $\phi_{n_{i}}\left(F_{n_{i}}\left(x_{n_{i}}\right)\right) \rightarrow w$ is any convergent subsequence then $w=\widehat{f}(z)$, which will establish the result.

Given such a subsequence, assume without loss of generality that $x_{n_{i}} \rightarrow x \in \Sigma$, and (using Lemma 97(c)) that $F_{n_{i}}\left(x_{n_{i}}\right)$ converges to some $y \in \Sigma$ with $\phi(y)=\phi(F(x))$. Since $\phi_{n_{i}}\left(x_{n_{i}}\right)=z$ for all $i$, it follows from Lemma 103 that $\phi(x)=z$. By the same lemma, $\phi_{n_{i}}\left(F_{n_{i}}\left(x_{n_{i}}\right)\right) \rightarrow \phi(y)=\phi(F(x))=\widehat{f}(\phi(x))=\widehat{f}(z)$ as required.

\section{References}

[1] L V Ahlfors, Conformal invariants: topics in geometric function theory, McGraw-Hill Series in Higher Mathematics, McGraw-Hill Book Co., New York (1973) MR0357743

[2] L V Ahlfors, L Sario, Riemann surfaces, Princeton Mathematical Series 26, Princeton Univ. Press (1960) MR0114911

[3] D Burago, Y Burago, S Ivanov, A course in metric geometry, Graduate Studies in Mathematics 33, American Mathematical Society (2001) MR1835418

[4] R Chamanara, F P Gardiner, N Lakic, A hyperelliptic realization of the horseshoe and baker maps, Ergodic Theory Dynam. Systems 26 (2006) 1749-1768 MR2279264

[5] A de Carvalho, Extensions, quotients and generalized pseudo-Anosov maps, from: "Graphs and patterns in mathematics and theoretical physics", Proc. Sympos. Pure Math. 73, Amer. Math. Soc., Providence, RI (2005) 315-338 MR2131019 
[6] A de Carvalho, T Hall, Unimodal generalized pseudo-Anosov maps, Geom. Topol. 8 (2004) 1127-1188 MR2087080

[7] A de Carvalho, T Hall, Paper surfaces and dynamical limits, Proc. Natl. Acad. Sci. USA 107 (2010) 14030-14035 MR2679493

[8] C J Earle, F P Gardiner, Teichmüller disks and Veech's $\mathcal{F}$-structures, from: "Extremal Riemann surfaces (San Francisco, CA, 1995)”, Contemp. Math. 201, Amer. Math. Soc., Providence, RI (1997) 165-189 MR1429199

[9] M K Fort, Jr, Mappings on $S^{1}$ into one-dimensional spaces, Illinois J. Math. 1 (1957) 505-508 MR0114190

[10] T Hall, The creation of horseshoes, Nonlinearity 7 (1994) 861-924 MR1275533

[11] K Kuratowski, Topology Vol II, Academic Press, New York (1968) MR0259835 New edition, revised and augmented. Translated from the French by A Kirkor

[12] O Lehto, K I Virtanen, Quasiconformal mappings in the plane, second edition, Grundl. Math. Wissen. 126, Springer, New York (1973) MR0344463 Translated from the German by K W Lucas

[13] J Milnor, W Thurston, On iterated maps of the interval, from: "Dynamical systems (College Park, MD, 1986-87)”, Lecture Notes in Math. 1342, Springer, Berlin (1988) 465-563 MR970571

[14] R L Moore, Concerning upper semi-continuous collections of continua, Trans. Amer. Math. Soc. 27 (1925) 416-428 MR1501320

[15] R L Moore, Foundations of point set theory, American Mathematical Society Colloquium Publications XIII, American Mathematical Society (1962) MR0150722

[16] J H Roberts, N E Steenrod, Monotone transformations of two-dimensional manifolds, Ann. of Math. 39 (1938) 851-862 MR1503441

[17] C A Rogers, Hausdorff measures, Cambridge Mathematical Library, Cambridge Univ. Press (1998) MR1692618 Reprint of the 1970 original. Foreword by K J Falconer

[18] S Smale, Differentiable dynamical systems, Bull. Amer. Math. Soc. 73 (1967) 747-817 MR0228014

[19] G T Whyburn, Analytic topology, American Mathematical Society Colloquium Publications XXVIII, American Mathematical Society (1963) MR0182943

Departamento de Matemática Aplicada, IME - USP

Rua do Matão 1010, Cidade Universitária, 05508-090 São Paulo, Brazil

Department of Mathematical Sciences, University of Liverpool

Liverpool L69 7ZL, UK

andre@ime.usp.br, t.hall@liv.ac.uk

Proposed: Danny Calegari

Received: 18 July 2011

Seconded: Leonid Polterovich, Yasha Eliashberg

Revised: 5 April 2012 